\title{
EVerT: cryotherapy versus salicylic acid for the treatment of verrucae - a randomised controlled trial
}

S Cockayne, M Curran, G Denby, F Hashmi, C Hewitt, K Hicks, S Jayakody, A Kang'ombe, C McIntosh, N McLarnon, E Stamuli, K Thomas, G Turner, D Torgerson and I Watt on behalf of the EVerT team

Health Technology Assessment NIHR HTA programme www.hta.ac.uk 


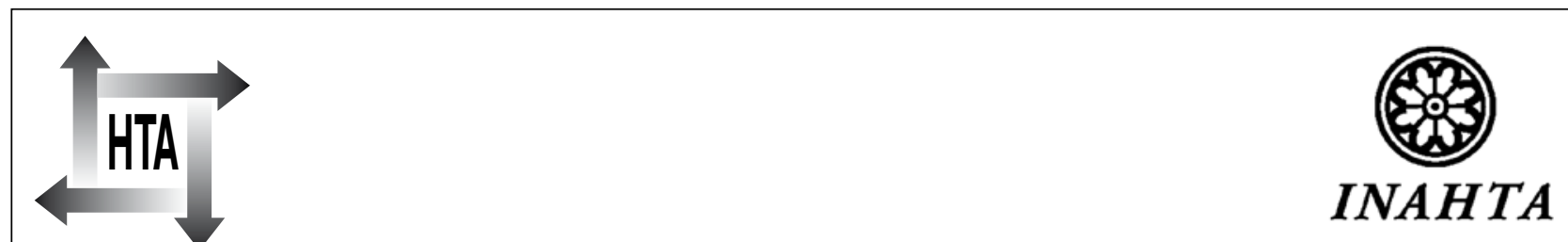

\section{How to obtain copies of this and other HTA programme reports}

An electronic version of this title, in Adobe Acrobat format, is available for downloading free of charge for personal use from the HTA website (www.hta.ac.uk). A fully searchable DVD is also available (see below).

Printed copies of HTA journal series issues cost $£ 20$ each (post and packing free in the UK) to both public and private sector purchasers from our despatch agents.

Non-UK purchasers will have to pay a small fee for post and packing. For European countries the cost is $£ 2$ per issue and for the rest of the world £3 per issue.

How to order:

- fax (with credit card details)

- post (with credit card details or cheque)

- phone during office hours (credit card only).

Additionally the HTA website allows you to either print out your order or download a blank order form.

\section{Contact details are as follows:}

Synergie UK (HTA Department)

Digital House, The Loddon Centre

Wade Road

Basingstoke

Hants RG24 8QW

\section{Email: orders@hta.ac.uk}

Tel: 08458124000 - ask for 'HTA Payment Services'

(out-of-hours answer-phone service)

Fax: 08458124001 - put 'HTA Order' on the fax header

\section{Payment methods}

Paying by cheque

If you pay by cheque, the cheque must be in pounds sterling, made payable to University of Southampton and drawn on a bank with a UK address.

Paying by credit card

You can order using your credit card by phone, fax or post.

\section{Subscriptions}

NHS libraries can subscribe free of charge. Public libraries can subscribe at a reduced cost of $£ 100$ for each volume (normally comprising 40-50 titles). The commercial subscription rate is $£ 400$ per volume (addresses within the UK) and $£ 600$ per volume (addresses outside the UK). Please see our website for details. Subscriptions can be purchased only for the current or forthcoming volume.

\section{How do I get a copy of HTA on DVD?}

Please use the form on the HTA website (www.hta.ac.uk/htacd/index.shtml). HTA on DVD is currently free of charge worldwide.

The website also provides information about the HTA programme and lists the membership of the various committees. 


\title{
EVerT: cryotherapy versus salicylic acid for the treatment of verrucae - a randomised controlled trial
}

\author{
S Cockayne, ${ }^{1 *}$ M Curran, ${ }^{2}$ G Denby, ${ }^{2}$ F Hashmi, ${ }^{3}$ \\ C Hewitt, ${ }^{1}$ K Hicks, ${ }^{1}$ S Jayakody, ${ }^{1}$ A Kang'ombe, ${ }^{1}$ \\ C McIntosh, ${ }^{4} \mathrm{~N}$ McLarnon, ${ }^{5} \mathrm{E}$ Stamuli, ${ }^{1} \mathrm{~K}$ Thomas, ${ }^{6}$ \\ G Turner, ${ }^{1} \mathrm{D}$ Torgerson ${ }^{1}$ and I Watt ${ }^{1,7}$ on behalf of the \\ EVerT team
}

1Department of Health Sciences, York Trials Unit, University of York, York, UK ${ }^{2}$ School of Health, University of Northampton, Northampton, UK ${ }^{3}$ University of Brighton, School of Health Professions, Division of Podiatry, Eastbourne, UK

${ }^{4}$ The National University of Ireland Galway, School of Podiatry, Galway, Ireland ${ }^{5}$ Glasgow Caledonian University, School of Health and Social Care, Glasgow, UK ${ }^{6}$ Centre of Evidence Based Dermatology, University of Nottingham, Nottingham, UK ${ }^{7}$ Hull York Medical School, University of York, York, UK

${ }^{*}$ Corresponding author

Declared competing interests of authors: The $50 \%$ salicylic acid (Verrugon) plasters and felt pads were provided to the University of York, free of charge, by the manufacturer William Ransom \& Son Plc. BOC Cryospeed provided liquid nitrogen storage equipment at reduced cost. Neither company has had any input into the design, analysis and reporting of the study.

Published September 2011

DOI: $10.3310 /$ hta15320

This report should be referenced as follows:

Cockayne S, Curran M, Denby G, Hashmi F, Hewitt C, Hicks K, et al. EVerT: cryotherapy versus salicylic acid for the treatment of verrucae - a randomised controlled trial. Health Technol Assess 2011;15(32).

Health Technology Assessment is indexed and abstracted in Index Medicus/MEDLINE, Excerpta Medica/EMBASE, Science Citation Index Expanded (SciSearch ${ }^{\circledR}$ ) and Current Contents ${ }^{\circledast} /$ Clinical Medicine. 
The Health Technology Assessment (HTA) programme, part of the National Institute for Health Research (NIHR), was set up in 1993. It produces high-quality research information on the effectiveness, costs and broader impact of health technologies for those who use, manage and provide care in the NHS. 'Health technologies' are broadly defined as all interventions used to promote health, prevent and treat disease, and improve rehabilitation and long-term care.

The research findings from the HTA programme directly influence decision-making bodies such as the National Institute for Health and Clinical Excellence (NICE) and the National Screening Committee (NSC). HTA findings also help to improve the quality of clinical practice in the NHS indirectly in that they form a key component of the 'National Knowledge Service'.

The HTA programme is needs led in that it fills gaps in the evidence needed by the NHS. There are three routes to the start of projects.

First is the commissioned route. Suggestions for research are actively sought from people working in the NHS, from the public and consumer groups and from professional bodies such as royal colleges and NHS trusts. These suggestions are carefully prioritised by panels of independent experts (including NHS service users). The HTA programme then commissions the research by competitive tender.

Second, the HTA programme provides grants for clinical trials for researchers who identify research questions. These are assessed for importance to patients and the NHS, and scientific rigour.

Third, through its Technology Assessment Report (TAR) call-off contract, the HTA programme commissions bespoke reports, principally for NICE, but also for other policy-makers. TARs bring together evidence on the value of specific technologies.

Some HTA research projects, including TARs, may take only months, others need several years. They can cost from as little as $£ 40,000$ to over $£ 1$ million, and may involve synthesising existing evidence, undertaking a trial, or other research collecting new data to answer a research problem.

The final reports from HTA projects are peer reviewed by a number of independent expert referees before publication in the widely read journal series Health Technology Assessment.

Criteria for inclusion in the HTA journal series

Reports are published in the HTA journal series if (1) they have resulted from work for the HTA programme, and (2) they are of a sufficiently high scientific quality as assessed by the referees and editors.

Reviews in Health Technology Assessment are termed 'systematic' when the account of the search, appraisal and synthesis methods (to minimise biases and random errors) would, in theory, permit the replication of the review by others.

The research reported in this issue of the journal was commissioned by the HTA programme as project number 05/513/02. The contractual start date was in October 2006. The draft report began editorial review in July 2010 and was accepted for publication in January 2011. As the funder, by devising a commissioning brief, the HTA programme specified the research question and study design. The authors have been wholly responsible for all data collection, analysis and interpretation, and for writing up their work. The HTA editors and publisher have tried to ensure the accuracy of the authors' report and would like to thank the referees for their constructive comments on the draft document. However, they do not accept liability for damages or losses arising from material published in this report.

The views expressed in this publication are those of the authors and not necessarily those of the HTA programme or the Department of Health.

Editor-in-Chief:

Series Editors:

Professor Tom Walley CBE

Dr Martin Ashton-Key, Professor Aileen Clarke, Dr Tom Marshall, Professor John Powell, Dr Rob Riemsma and Professor Ken Stein

Associate Editor:

Dr Peter Davidson

Editorial Contact: edit@southampton.ac.uk

ISSN 1366-5278 (Print)

ISSN 2046-4924 (Online)

ISSN 2046-4932 (DVD)

(c) Queen's Printer and Controller of HMSO 2011. This work was produced by Cockayne et al. under the terms of a commissioning contract issued by the Secretary of State for Health.

This journal is a member of and subscribes to the principles of the Committee on Publication Ethics (COPE) (http://www. publicationethics.org/).

This journal may be freely reproduced for the purposes of private research and study and may be included in professional journals provided that suitable acknowledgement is made and the reproduction is not associated with any form of advertising. Applications for commercial reproduction should be addressed to: NETSCC, Health Technology Assessment, Alpha House, University of Southampton Science Park, Southampton SO16 7NS, UK.

Published by Prepress Projects Ltd, Perth, Scotland (www.prepress-projects.co.uk), on behalf of NETSCC, HTA.

Printed on acid-free paper in the UK by the Charlesworth Group. 


\title{
Abstract
}

\section{EVerT: cryotherapy versus salicylic acid for the treatment of verrucae - a randomised controlled trial}

\author{
S Cockayne, ${ }^{1 *}$ M Curran, ${ }^{2}$ G Denby, ${ }^{2}$ F Hashmi, ${ }^{3}$ C Hewitt, ${ }^{1}$ K Hicks, ${ }^{1}$ \\ S Jayakody, ${ }^{1}$ A Kang'ombe, ${ }^{1}$ C Mclntosh, ${ }^{4} \mathrm{~N}$ McLarnon, ${ }^{5}$ E Stamuli, ${ }^{1}$ \\ K Thomas, ${ }^{6} \mathrm{G}$ Turner, ${ }^{1} \mathrm{D}$ Torgerson ${ }^{1}$ and I Watt ${ }^{1,7}$ on behalf of the \\ EVerT team
}

${ }^{1}$ Department of Health Sciences, York Trials Unit, University of York, York, UK ${ }^{2}$ School of Health, University of Northampton, Northampton, UK

${ }^{3}$ University of Brighton, School of Health Professions, Division of Podiatry, Eastbourne, UK

${ }^{4}$ The National University of Ireland Galway, School of Podiatry, Galway, Ireland ${ }^{5}$ Glasgow Caledonian University, School of Health and Social Care, Glasgow, UK ${ }^{6}$ Centre of Evidence Based Dermatology, University of Nottingham, Nottingham, UK ${ }^{7}$ Hull York Medical School, University of York, York, UK

${ }^{*}$ Corresponding author sarah.cockayne@york.ac.uk

Objective: To compare the clinical effectiveness and cost-effectiveness of cryotherapy using liquid nitrogen versus patient daily self-treatment with $50 \%$ salicylic acid for the treatment of verrucae (plantar warts).

Design: A multicentre, pragmatic, open, two-armed randomised controlled trial with an economic evaluation. Randomisation was simple, with the allocation sequence generated by a computer in a $1: 1$ ratio.

Setting: Podiatry clinics, university podiatry schools and primary care in England, Scotland and Ireland.

Participants: Patients were eligible if they presented with a verruca which, in the opinion of the health-care professional, was suitable for treatment with both salicylic acid and cryotherapy, and were aged 12 years and over.

Interventions: Cryotherapy using liquid nitrogen delivered by a health-care professional compared with daily patient self-treatment with $50 \%$ salicylic acid (Verrugon, William Ransom \& Son PIc, Hitchin, UK) for a maximum of 8 weeks.

Main outcome measures: The primary outcome was complete clearance of all verrucae at 12 weeks. Secondary outcomes were complete clearance of all verrucae at 12 weeks, controlling for age, whether or not the verrucae had been previously treated and type of verrucae, with a second model to explore the effect of patient preferences, time to clearance of verrucae, clearance of verrucae at 6 months, number of verrucae at 12 weeks and patient satisfaction with the treatment.

Results: In total, 240 eligible patients were recruited, with 117 patients allocated to the cryotherapy group and 123 to the salicylic acid group. There was no evidence of a difference in clearance rates between the treatment groups in the primary outcome [17/119 $(14.3 \%)$ in the salicylic acid group vs $15 / 110(13.6 \%)$ in the cryotherapy group; $p=0.89$ ]. The results of the study did not change when controlled for age, whether or not the verrucae had been previously treated and type of verrucae, or when patient preferences were explored. There was no evidence of a difference in time to clearance of verrucae 
between the two groups [hazard ratio (HR) $0.80,95 \%$ confidence interval $(\mathrm{Cl}) 0.51$ to 1.25 ; $p=0.33$ ] or in the clearance of verrucae at 6 months (33.7\% cryotherapy vs $30.5 \%$ salicylic acid). There was no evidence of a difference in the number of verrucae at 12 weeks between the two groups (incidence rate ratio $1.08,95 \% \mathrm{Cl} 0.81$ to $1.43 ; p=0.62$ ). Nineteen participants reported 28 adverse events, 14 in each group, with two treatment-related non-serious adverse events in the cryotherapy group. Cryotherapy was also associated with higher mean costs per additional healed patient ( $£ 101.17,95 \%$ bias-corrected and accelerated $\mathrm{Cl} £ 85.09$ to $£ 117.26)$. The probability of cryotherapy being cost-effective is $40 \%$ for a range of willingness-to-pay thresholds of $£ 15,000-30,000$ per patient healed. Conclusions: There is no evidence for a difference in terms of clearance of verrucae between cryotherapy and salicylic acid (at both 12 weeks and 6 months), number of verrucae at 12 weeks and time to clearance of verrucae. Cryotherapy was associated with higher mean costs per additional healed patient compared with salicylic acid.

Trial registration: Current Controlled Trials ISRCTN18994246.

Funding: This project was funded by the NIHR Health Technology Assessment programme and will be published in full in Health Technology Assessment; Vol. 15, No. 32. See the HTA programme website for further project information. 


\section{Contents}

List of abbreviations vii

$\begin{array}{ll}\text { Executive summary ix } & \text { ix }\end{array}$

1. Background 1

What are verrucae? 1

What treatments are available? 1

What evidence is there for the most commonly used treatments? 2

Why did we do the trial?

Specific objectives of the trial 2

2. Methods 3

Trial design 3

Approvals obtained 3

Trial sites 3

Participant eligibility 3

Recruitment into the trial 4

Baseline assessment 5

Ineligible patients 6

Randomisation 6

Sample size $\quad 6$

Trial interventions $\quad 7$

Participant follow-up 8

Trial completion 8

Measurement of primary outcome 8

Measurement of secondary outcomes 9

Additional data collected 9

Statistical analysis 11

Economic analysis 12

3. Protocol changes 19

$\begin{array}{lr}\text { Inclusion and exclusion criteria } & 19\end{array}$

$\begin{array}{lr}\text { Treatment regimens } & 19\end{array}$

Clarification of secondary outcomes and analysis 19

Questionnaire response rates $\quad 20$

$\begin{array}{ll}\text { Recruitment } & 20\end{array}$

4. Clinical results section $\quad 21$

$\begin{array}{ll}\text { Trial recruitment } & 21\end{array}$

Baseline participant characteristics $\quad 21$

Primary outcome: complete clearance of verrucae at 12 weeks 25

Secondary outcomes $\quad 26$

$\begin{array}{ll}\text { Additional data collected } & 27\end{array}$

5. Economic analysis 33

Summary of the resource usage 33

Missing data on resource use and outcome 33 
Data analysis $\quad 34$

Summary of findings 38

6. Discussion 41

Key findings $\quad 41$

Comparison with other studies/reviews $\quad 41$

Treatment regimen $\quad 42$

Patient satisfaction with treatment 43

Strengths and limitations of the study 43

Generalisability of the results 44

Implications for health care $\quad 45$

Implications for research 45

$\begin{array}{lr}\text { Acknowledgements } & 47\end{array}$

$\begin{array}{lr}\text { References } & 49\end{array}$

Appendix 1 Regulatory approvals 51

Appendix 2 Details of the study sites 53

Appendix 3 Patient information sheets and consent form 55

$\begin{array}{ll}\text { Appendix } 4 \text { Data collection forms } & 69\end{array}$

$\begin{array}{ll}\text { Appendix } 5 \text { Advertising material } & 115\end{array}$

$\begin{array}{ll}\text { Appendix } 6 \text { Flow chart for EVerT trial } & 135\end{array}$

$\begin{array}{ll}\text { Appendix } 7 \text { Trial protocol } & 137\end{array}$

$\begin{array}{ll}\text { Health Technology Assessment programme } & 165\end{array}$ 


\section{List of abbreviations}

\begin{tabular}{|c|c|}
\hline BCA & bias-corrected and accelerated \\
\hline CE plane & cost-effectiveness plane \\
\hline CEAC & cost-effectiveness acceptance curve \\
\hline $\mathrm{CI}$ & confidence interval \\
\hline CONSORT & Consolidated Standards of Reporting Trials \\
\hline CTA & clinical trial authorisation \\
\hline df & degrees of freedom \\
\hline DMEC & Data Monitoring and Ethics Committee \\
\hline EVerT & Effective Verruca Treatments \\
\hline GP & general practitioner \\
\hline HR & hazard ratio \\
\hline HTA & Health Technology Assessment \\
\hline ICER & incremental cost-effectiveness ratio \\
\hline IQR & interquartile range \\
\hline IRR & incidence rate ratio \\
\hline ISRCTN & International Standard Randomised Controlled Trial Number \\
\hline LREC & Local Research Ethics Committee \\
\hline MHRA & Medicines and Healthcare Products Regulatory Agency \\
\hline MREC & Multicentre Research Ethics Committee \\
\hline NIHR & National Institute for Health Research \\
\hline NUI & National University of Ireland \\
\hline OR & odds ratio \\
\hline OTC & over-the-counter \\
\hline PCT & primary care trust \\
\hline PSSRU & Personal Social Services Research Unit \\
\hline RCT & randomised controlled trial \\
\hline SD & standard deviation \\
\hline SE & standard error \\
\hline TSC & Trial Steering Committee \\
\hline YTU & York Trials Unit \\
\hline
\end{tabular}

All abbreviations that have been used in this report are listed here unless the abbreviation is well known (e.g. NHS), or it has been used only once, or it is a non-standard abbreviation used only in figures/tables/appendices, in which case the abbreviation is defined in the figure legend or in the notes at the end of the table. 



\section{Executive summary}

\section{Objective}

To compare the clinical effectiveness and cost-effectiveness of cryotherapy using liquid nitrogen versus $50 \%$ salicylic acid for the treatment of verrucae (plantar warts).

\section{Methods}

\section{Design}

A multicentre, pragmatic, open, two-armed randomised controlled trial was undertaken with an economic evaluation. Participants were randomised using simple randomisation, with the allocation sequence generated by a computer in a 1:1 ratio. The sample size calculation was based on the difference in cure rates at 12 weeks between the two groups. In order to give $80 \%$ power to show a difference in cure rates of $70 \%$ versus $85 \%$ required 120 patients in each group or 133 patients after allowing for $10 \%$ attrition, i.e. a total of 266 .

\section{Setting}

Participants were recruited from 14 sites in England, Scotland and Ireland: two podiatry clinics, one of which was in Scotland, four university podiatry schools, one of which was in Ireland and eight general practitioner (GP) practices in five different regions of England.

\section{Participants}

Potential participants were identified by a health-care professional from the study site from GP referrals or self-referrals received by the podiatry or GP practice for the treatment of verrucae. Patients were eligible to participate in the trial if they presented with a verruca that, in the opinion of the health-care professional, was suitable for treatment with both salicylic acid and cryotherapy, and were aged 12 years and over.

\section{Interventions}

Participants randomised to cryotherapy using liquid nitrogen received a maximum of four treatments, 14-21 days apart, delivered by a health-care professional. The first treatment was a gentle freeze lasting approximately 10 seconds, with subsequent treatments undertaken according to the site's usual practice. Debridement, masking and padding of the site were also undertaken according to the site's usual practice. Participants randomised to patient self-treatment with 50\% salicylic acid (Verrugon, William Ransom \& Son Plc, Hitchin, UK) were instructed on how to use the acid by a health-care professional and instructed to apply it once daily for a maximum of 8 weeks.

\section{Main outcome measures}

The primary outcome was complete clearance of all verrucae at 12 weeks. Secondary outcomes were complete clearance of all verrucae at 12 weeks, controlling for age, whether or not the verrucae had been previously treated and type of verrucae, with a second model to explore the effect of patient preferences, time to clearance of verrucae, clearance of verrucae at 6 months, number of verrucae at 12 weeks and patient satisfaction with the treatment. 


\section{Results}

A total of 240 participants ( $90 \%$ of the sample size) were recruited to the trial, with 117 patients allocated to the cryotherapy group and 123 to the salicylic acid group. There was no evidence of a difference between the proportions of participants with complete clearance of all verrucae at 12 weeks between the salicylic acid and cryotherapy groups $\{14.3 \%$ vs $13.6 \%$, chi-squared test statistic 0.02 [ 1 degrees of freedom (df)]; $p=0.89$ \}. Cryotherapy was also associated with higher mean costs per additional healed patient $[\mathfrak{E} 101.17,95 \%$ bias-corrected and accelerated confidence interval (CI) $£ 85.09$ to $£ 117.26$ ]. The probability of cryotherapy being cost-effective is $40 \%$ for a range of willingness-to-pay thresholds of $£ 15,000-30,000$ per patient healed. The results of the study did not change when the analysis was repeated but controlled for age, whether or not the verrucae had been previously treated and type of verrucae or patients' preferences at baseline.

There was no evidence of a difference in the clearance of verrucae at 6 months between the salicylic acid and the cryotherapy groups [30.5\% vs 33.7\%, chi-squared test statistic 0.22 ( $1 \mathrm{df}$ ); $p=0.64$ ] nor in time to clearance between the two groups [hazard ratio (HR) $0.80,95 \%$ CI 0.51 to $1.25 ; p=0.33$ ]. There was no evidence of a difference in the number of verrucae at 12 weeks between the two groups (incidence rate ratio $1.10,95 \%$ CI 0.84 to $1.45 ; p=0.47$ ).

\section{Conclusions}

There was no evidence of a difference in clearance rates of verrucae between the $50 \%$ salicylic acid and the cryotherapy using liquid nitrogen groups. However, the results of this study are applicable only to verrucae or plantar warts and not to warts at other sites, such as the hands, which may respond differently to cryotherapy.

The findings of this study would not be generalisable to other freezing agents, such as nitrous oxide or over-the-counter (OTC) freezing treatments, as they freeze at a higher temperature than liquid nitrogen. Nor could the results be extrapolated to other concentrations of salicylic acid available as OTC preparations, which are usually of a lower concentration, or to the treatment being applied by a health-care professional.

Cryotherapy is associated with higher mean costs per patient healed compared to salicylic acid. Both higher mean costs and lack of evidence of a difference in effectiveness result in cryotherapy having a low probability of being cost-effective, even at high $(>£ 15,000$ per patient healed) costeffectiveness threshold values.

\section{Implications for future research}

There are other treatments available for cutaneous warts, but with very little good-quality evidence assessing their effectiveness. The effectiveness of these treatments is worthy of further study.

\section{Trial registration}

This trial is registered as ISRCTN18994246.

\section{Funding}

This project was funded by the NIHR Health Technology Assessment programme and will be published in full in Health Technology Assessment; Vol. 15, No. 32. See the HTA programme website for further project information. 


\section{Chapter 1}

\section{Background}

\section{What are verrucae?}

Verrucae (or plantar warts) are caused by the human papillomavirus. They are extremely common, being experienced by most people at some time during their lives. Verrucae are infectious and can be painful, especially when affecting the soles of the feet or the nails. Although most verrucae will spontaneously disappear without treatment, many patients seek treatment because they are painful or because they are being prevented from doing sports and other activities of daily living.

Various studies have examined the prevalence of warts/verrucae and have produced a wide range of estimates. Three population-based studies reported point prevalence rates ranging from $0.84 \%$ (USA) $)^{1}$ to $3.3 \%(\mathrm{UK})^{2}$ and up to $12.9 \%$ (Russian Federation). ${ }^{3}$ Studies of school-age populations have reported prevalence of $12 \%$ in 4 to 6 -year-olds, $3.9 \%$ to $4.7 \%$ in 11 to 16 -year-olds ${ }^{4}$ and $24 \%$ in 16 to 18 -year-olds. ${ }^{5}$ A recent cross-sectional study, including 1465 children in four primary schools in the Netherlands, reported prevalence rates in children aged $4-12$ years of $33 \%$ ( $9 \%$ had hand warts, $20 \%$ had plantar warts and $4 \%$ had both). ${ }^{6}$

Estimates of the rate of natural resolution of warts vary widely. Massing ${ }^{7}$ found that two-thirds resolved within 2 years, but the resolution rates reported in the placebo arms of trials suggest that warts may resolve more rapidly. In a Cochrane systematic review ${ }^{8}$ of wart treatment, 21 trials with placebo groups were reviewed. The average proportion that were clear of warts in the placebo groups in these trials was $27 \%$ (range 0 to $73 \%$ ), after an average period of 15 weeks (range 4 to 24 weeks). This has led some to suggest that warts should not be treated at all.9,10 However, some viral warts may persist for many years and there is no reliable means of predicting which ones will resolve spontaneously.

Verrucae are spread by direct skin-to-skin contact or indirectly via contact with contaminated surfaces (e.g. swimming pools or communal showers), ${ }^{11}$ although having a family member with a wart and having a high incidence of warts within a child's class have been shown to be stronger risk factors than the use of swimming pools and shared bathing areas. ${ }^{6}$ If a verruca is scratched or knocked it can bleed, making it easier for the virus to infect another part of the body through a breach in the skin. ${ }^{12}$

\section{What treatments are available?}

Many treatments are available for the treatment of verrucae, including cryotherapy, topically applied treatments, surgical curettage, and complementary and alternative therapies. The most commonly prescribed treatments are cryotherapy with liquid nitrogen and topical salicylic acid. ${ }^{13}$

Side-effects are common with all verrucae treatments, and include pain, burning, blistering, bleeding and scarring. Pain and blistering are more commonly reported for cryotherapy treatments, ${ }^{14}$ and, for this reason, cryotherapy is not recommended for young children. ${ }^{10}$ 


\section{What evidence is there for the most commonly used treatments?}

A Cochrane systematic review ${ }^{8}$ that assessed the effects of different local treatments of cutaneous, non-genital warts was updated in 2006 (search date March 2005). This review highlighted considerable uncertainty around the optimal treatment of verrucae.

The best available evidence was for topical treatments containing salicylic acid (of varied strengths). These preparations were significantly better than placebo. Data pooled from five placebo-controlled trials showed a cure rate of 117/160 (73\%) compared with 78/162 (48\%) in control subjects. ${ }^{8}$

Evidence for the effectiveness of cryotherapy was limited. The review found two trials comparing cryotherapy with salicylic acid and one comparing duct tape with cryotherapy. These trials showed no significant difference in efficacy for the compared treatments. More recently, a headto-head trial of salicylic acid compared with cryotherapy has been reported in a primary care setting in the Netherlands. This trial found that cryotherapy was significantly better than salicylic acid for the treatment of hand warts, but that there was no significant benefit of cryotherapy compared with salicylic acid in plantar warts. ${ }^{15}$ Cure rates for plantar warts were $29 \%$ for cryotherapy, $33 \%$ for salicylic acid and $23 \%$ for a no-treatment control group.

\section{Why did we do the trial?}

The treatment of warts and verrucae represents a considerable cost burden to both patients and the NHS. An economic decision model assessing the effectiveness and cost-effectiveness of salicylic acid and cryotherapy estimated that almost 2 million people in England and Wales see their general practitioner (GP) for the treatment of cutaneous warts each year, at a cost of at least $£ 40 \mathrm{M}$ per annum. ${ }^{14}$

Despite this, the evidence base on which to inform clinical decision-making is poor. Of the 60 trials identified in the 2006 Cochrane systematic review, ${ }^{8} 46$ (77\%) were classified as low quality; in addition, heterogeneity between the trials was high and analyses were often inappropriate or misleading. A major conclusion from the Cochrane review $^{8}$ was that a trial comparing topical salicylic acid with cryotherapy was urgently needed.

In response to an open call for trial proposals looking at medicines for children, the National Institute for Health Research (NIHR) Health Technology Assessment (HTA) programme agreed to fund the EVerT (Effective Verruca Treatments) trial, with the aim of establishing the efficacy and cost-effectiveness of these two treatments.

\section{Specific objectives of the trial}

- To assess the clinical effectiveness and acceptability of cryotherapy compared with salicylic acid for the treatment of verrucae.

- To assess the cost-effectiveness of the compared treatments. 


\section{Chapter 2}

\section{Methods}

\section{Trial design}

The EVerT trial was an open, pragmatic, multicentred, two-armed, randomised controlled trial (RCT) with equal randomisation. Participants with verrucae were randomised $(1: 1)$ to receive either:

- cryotherapy using liquid nitrogen, delivered by a health-care professional (a podiatrist, practice nurse or GP) or

- once-daily self-treatment with 50\% salicylic acid (Verrugon, William Ransom \& Son Plc, Hitchin, UK).

\section{Approvals obtained}

The Trent Multicentre Research Ethics Committee (MREC) approved the study and substantial amendment to address the NIHR HTA programme reviewers' comments on 26 October 2004 and 16 August 2006, respectively. Galway Research Ethics Committee approved the study on 20 March 2009.

Salicylic acid was classified as a medicinal product, therefore, clinical trial authorisations (CTAs) were obtained from the competent authorities in the UK and Ireland: the Medicines and Healthcare products Regulatory Agency (MHRA) (CTA number 22803/0001/001-0001) on 8 February 2005 and the Irish Medicines Board (clinical trial number CT 1552/1/1 Salicylic Acid/ Liquid Nitrogen) on 30 January 2009.

The details of MREC, local research ethics committees (LRECs), competent authorities and research and development department approvals are provided in Appendix 1.

The trial was assigned the International Standard Randomised Controlled Trial Number (ISRCTN) of ISRCTN18994246; EudraCT number 2004-000905-24; and National Research Register number N0484189151.

\section{Trial sites}

The study was conducted in 16 study sites: 15 in the UK and 1 in Ireland. Sites were recruited throughout the duration of the trial. The sites were podiatry schools, outpatient podiatry clinics, GP practices or, in one case, a primary care trust (PCT) podiatry service outpatient clinic. Details of the study sites are provided in Appendix 2.

\section{Participant eligibility}

People with one or more verrucae were recruited into this study. 


\section{Inclusion criteria}

Potential participants were eligible for inclusion in the trial if they met the following criteria:

- They had a verruca which, in the opinion of the health-care professional, was suitable for treatment with both salicylic acid and cryotherapy.

- They were aged 12 years and over.

The study was funded via the NIHR HTA's medicines for children call. Consequently, the initial inclusion criteria focused on children and young people between the ages of 12 and 24 years, inclusive. However, because of poor recruitment the upper age restriction was lifted.

\section{Exclusion criteria}

Potential participants were excluded if they met one or more of the following criteria:

- They were currently in a trial evaluating other treatments for their verruca.

- They had impaired healing, for example owing to diabetes, peripheral vascular disease or any other condition.

- They were immunosuppressed, for example had agammaglobulinaemia or were taking immunosuppressant drugs such as oral corticosteroids.

- They had neuropathy.

- They were currently on renal dialysis.

- They had cold intolerance, for example Raynaud syndrome or cold urticaria.

- They had any of the following conditions: blood dyscrasias of unknown origin, cryoglobulinaemia, cryofibrinogenaemia or collagen or autoimmune disease.

- They were unable to give informed consent.

\section{Recruitment into the trial}

Members of the research team participating in the study received 'Good Clinical Practice' training, as well as training in all aspects of the trial, including participant recruitment, eligibility criteria, trial protocol, adverse event reporting procedures and trial documentation. In order to standardise the study prior to commencement, each study site also received a trial handbook.

Potential participants for the trial were identified by a health-care professional at the study site from GP referrals, or self-referrals received by the podiatry clinic or GP practice for the treatment of verrucae. Participants were provided with an appointment for assessment/treatment and sent an invitation letter, information sheet about the trial, baseline questionnaire and consent form for the study (see Appendices 3 and 4). The flow of participants through the trial is presented in a CONSORT (Consolidated Standards of Reporting Trials) diagram (see Figure 2).

In order to aid recruitment, one or more of the following strategies were adopted at some sites to increase the number of people with verrucae presenting to the clinics:

- GPs in the recruiting area were approached by either the York Trials Unit (YTU: University of York, UK) or the local Primary Care Research Network. They were requested to refer patients presenting with a verruca and who expressed an interest in taking part in the trial to the recruiting site.

- The trial was promoted by means of a recruitment poster that was displayed in 41 libraries, 15 pharmacies, 19 swimming pools, 8 supermarkets, 2 universities and 2 hospitals. 
- Secondary schools were approached and asked to send out study information to their students. Fifteen schools in three different recruiting areas agreed to send out study information to 7410 students and displayed recruitment posters.

- The trial was publicised in two local newspapers, in three university press releases, on three university websites and on two local radio stations. Potential participants were directed to the local recruiting site.

The documentation used to aid recruitment to the study is included in Appendix 5.

For individuals responding to an advert for trial participants, telephone screening by the study sites was recommended to ensure that the potential participants fulfilled the inclusion criteria.

Participants were given a minimum of 24 hours to read the information sheet and consider participation. In Ireland, where possible, there was a minimum of 6 days between the patient signing the consent form and the start of treatment in order to comply with local regulations. Participants who wished to take part in the study and who returned their baseline questionnaire were screened by the health-care professional using a randomisation form that listed the eligibility criteria (see Appendix 4). Eligible patients, and their parent/guardian for those under 16 years of age, were able to discuss the study in more detail prior to providing written informed consent. Baseline data were then recorded and a digital photograph taken of the verruca(e). Participants' GPs were notified of their involvement in the EVerT trial after recruitment.

\section{Baseline assessment}

After written informed consent had been obtained, baseline data were collected using the podiatrist treatment assessment form and the baseline questionnaire (see Appendix 4). The following data were collected.

\section{Type and number of verruca(e)}

The number and type of verruca(e) (mosaic or non-mosaic) were collected on the podiatrist treatment assessment form in order to examine whether or not mosaic verrucae respond less well to treatment than simple verrucae.

\section{Duration and previous treatment of current verruca(e)}

The duration of the current verruca(e) and type of any previous treatment received were recorded on the participant baseline questionnaire.

\section{Reason for seeking treatment}

The reasons for seeking treatment for the verrucae were recorded on the participant baseline questionnaire.

\section{Level of pain}

Participants were asked to rate how painful their current verruca was at baseline on a five-point Likert scale of $0-4$, where 0 was not at all painful and 4 was extremely painful.

\section{Number of previous verrucae and age at which they occurred}

The number of previous verrucae and age at which they occurred were recorded on the participant baseline questionnaire. 


\section{Patient's treatment preference}

The patient's treatment preference was recorded on the podiatrist treatment assessment form to allow us to explore the influence of the patient's treatment preference on treatment outcomes.

\section{Date of birth}

Date of birth was recorded on the participant baseline questionnaire, allowing age at recruitment to be calculated and to allow us to explore the influence of the participant's age on treatment outcomes.

\section{Gender}

The gender of participants was recorded on the participant baseline questionnaire.

\section{Ineligible patients}

The health-care professionals were asked to complete an ineligible patient form (see Appendix 4) for those participants who wished to take part in the trial, but were ineligible to do so. Data collected on this form were reasons why the patient was not eligible, date of birth, gender, type of wart and date of consideration for trial entry. Where the patient was willing, a completed baseline questionnaire was also collected.

\section{Randomisation}

Patients were randomised equally between the two treatment arms: cryotherapy using liquid nitrogen delivered by the health-care professional (podiatrist, practice nurse and GP) or daily self-treatment by the patient with $50 \%$ salicylic acid. The health-care professional at the recruiting site randomised the patient using the secure, remote, independent YTU telephone or web-based randomisation service. Randomisation was simple, i.e. it was not restricted in any way. Stratified randomisation was not used in order to reduce the risk of subversion, which can occur using forms of restricted randomisation. The allocation sequence was computer generated, with the treatment allocation being concealed from both the health-care professional and YTU until the moment of randomisation.

\section{Sample size}

The Cochrane systematic review ${ }^{8}$ found only one small trial directly comparing the effectiveness of a chemical treatment, salicylic acid, with cryotherapy in patients with warts on their feet alone. This poor-quality study found a $58 \%$ cure rate among the patients allocated to cryotherapy compared with $41 \%$ among those treated with salicylic acid. This difference of $17 \%$ was not statistically significant. The overall cure rates from this study are smaller than those observed in two placebo-controlled trials of salicylic acid, both of which reported cure rates of $85 \%$ for active treatment, possibly because more resistant verrucae were included in the study comparing cryotherapy with salicylic acid. The EVerT trial was powered to show a $15 \%$ difference in effectiveness. To give us $80 \%$ power (5\% two-sided significance) to show a difference in cure rates of $70 \%$ versus $85 \%$ at 12 weeks, we required a sample size of 120 patients in each treatment group or 133 patients in each group after allowing for $10 \%$ attrition (i.e. 266 in total). 


\section{Trial interventions}

Participants were randomised to receive either cryotherapy using liquid nitrogen delivered by a health-care professional or daily self-treatment with 50\% salicylic acid (Verrugon).

\section{Cryotherapy using liquid nitrogen delivered by the health-care professional}

Patients randomised to cryotherapy using liquid nitrogen received up to a maximum of four treatments 14-21 days apart. Treatment was delivered by the health-care professional according to the usual practice of each trial site. Most of the health-care professionals delivering the cryotherapy had several years' experience in delivering cryotherapy using liquid nitrogen. If a patient presented with more than one verruca, the health-care professional was instructed to treat the verrucae as they would in normal practice.

Prior to treatment, if it was the site's normal practice, the callus surrounding the verruca(e) was debrided (e.g. with a scalpel or file) with any haemorrhages stopped by digital pressure only. The tissue surrounding the verruca was either masked (e.g. with petroleum jelly) or left unmasked, as per usual practice. Liquid nitrogen was applied using a spray (method of choice if available) or probe until the health-care professional was satisfied that the tissue had been frozen adequately. On the advice from the Trial Steering Committee (TSC), clinicians were advised that the first treatment should be a gentle freeze (approximately 10 seconds' duration) in order to ensure that the patient could tolerate the treatment. Silver nitrate was not applied to the verruca. If necessary, the health-care professional could pad the area surrounding the verruca after treatment, for example with $7 \mathrm{~mm}$ of felt-cavity padding. Patients were given a cryotherapy patient's advice sheet (see Appendix 3). Patients were also advised to keep the area dry for 24 hours and that the area may blister and be uncomfortable. If required, patients were recommended to use painkillers, as they would for a headache, if the area was very painful.

\section{Daily self-treatment by the patient with $50 \%$ salicylic acid}

Patients randomised to self-treatment with 50\% salicylic acid were instructed how to use the salicylic acid by the health-care professional and were provided with a salicylic acid patient's advice sheet (see Appendix 3) at the first trial appointment. Thereafter, the salicylic acid was applied once daily by the patient (or parent/guardian if appropriate) for a maximum of 8 weeks as per the manufacturer's instructions as follows:

- The self-adhesive ring should be fixed with the hole over the verruca.

- Squeeze a little Verrugon ointment into the hole and directly onto the verruca.

- Remove backing paper from plaster.

- Cover ring completely with plaster. Seal into position.

- Repeat treatment daily after gently pumicing or filing off the dead part of the verruca.

All patients were given a follow-up appointment at 2 weeks as a safety check. Further supplies of felt pads, plasters and salicylic acid were provided to the patient when required. Patients were asked to return all of the tubes of salicylic acid they had received during the trial to the treating health-care professional at their 12-week appointment. The health-care professional weighed the tube(s) to determine how much salicylic acid had been used over the 8-week period. 


\section{Participant follow-up}

Appendix 6 shows a summary of participant follow-up for the EVerT trial. Participants were given the option to complete participant questionnaires in either paper or web-based format according to their preference. In order to increase the response rate to the week-12 questionnaire, participants received an unconditional $£ 5$ ( $€ 5$ for the site in Ireland) with their week-12 questionnaire. The week-12 questionnaire was preceded by a letter notifying the participant that their week-12 questionnaire would arrive shortly and that it would be accompanied by a five pound (or five euro) note as an acknowledgement for their taking part in the trial and completing the questionnaires.

In order to minimise the difference in attendance between participants in the two groups, participants were reimbursed $£ 20$ for attending their week- 12 outcome assessment appointment with the health-care professional. Information about this reimbursement was included in the patient information sheet.

\section{Trial completion}

Participants were deemed to have exited the trial when:

- the participant had been in the trial for 6 months

- the participant wished to exit the trial fully

- the participant's health-care professional withdrew him/her from the trial

- the participant was lost to follow-up

- the participant died.

Instead of withdrawing fully from the trial, participants had the option of:

- withdrawing only from receiving the trial treatment

- withdrawing only from postal or web-based questionnaires

- withdrawing from the collection of data by the health-care professional

- any combination of the above.

If the participant elected to withdraw from all three (trial treatment, questionnaires and data collection) then he or she was deemed as a full withdrawal (trial exit). Health-care professionals were able to indicate any change in the patient's level of participation by completing the change of circumstances form (see Appendix 4). This ensured appropriate follow-up from the YTU.

\section{Measurement of primary outcome}

The primary outcome was complete clearance of all verrucae at 12 weeks after randomisation. Clearance of verrucae was defined as the restoration of normal skin on close inspection.

At the 12-week appointment the treating health-care professional or other member of the research team took a digital photograph of the participant's foot. Participants who did not attend their 12-week outcome assessment appointment were asked to take a digital photograph of their foot and send it to the YTU. Two blinded assessors independently assessed the photographs for 
each participant from all the sites to determine whether or not the verrucae had cleared, and whether or not they could tell which treatment the patient had received. The assessors discussed any discrepancies with referral to a third assessor for a final decision if required.

Previous studies co-ordinated by the YTU had found that using cameras to obtain blinded outcome assessments was not without its challenges. We therefore undertook an additional blinded outcome assessment at the recruiting site at the participant's 12-week appointment. This assessment would then be used in cases in which assessment of the digital photograph was not possible, for example when the photograph was not interpretable or was missing. The blinded outcome assessment at the site was undertaken by another member of the research team who was unaware of the treatment the participant had received. The health-care professional recorded whether or not the verruca(e) had completely cleared on the podiatrist outcome assessment form (see Appendix 4). Participants were reminded not to tell the person undertaking the blinded assessment which treatment they received and participants allocated to the salicylic acid group were asked not to return any used or unused Verrugon tubes to them. If the outcome assessment was not blinded, this was recorded on the podiatrist outcome assessment form.

The primary outcome was then calculated using whether or not the verrucae had cleared, as decided by the blinded assessors from the photographs. However, if no photographs were available for a participant, or if the photograph was not interpretable, then the outcome from the blinded assessment at 12 weeks was taken. If neither of these were available for a participant then the patient's self-reported outcome recorded in the week-12 questionnaire or on the 'verrucae gone' form (see Appendix 4) were used.

\section{Measurement of secondary outcomes}

\section{Self-reported time to clearance of verrucae}

Participants were asked to report if their verruca(e) had cleared on their week-3, week-12 and 6-month questionnaires (see Appendix 4) and, if it had cleared, on what date it cleared. In addition to this, participants were asked to return their 'verruca gone form' if their verrucae cleared at any other time points. If there was any discrepancy between the dates reported by the participant then the longest date to clearance was used.

\section{Clearance of verrucae at 6 months}

Clearance of verrucae at 6 months was recorded on the participant's 6-month questionnaire. If the participant had verrucae at 6 months then the position of the verrucae (either in the original or in a new position) was recorded.

\section{Number of verrucae remaining at the 12-week appointment}

The number of verrucae remaining at 12 weeks was recorded on the podiatrist outcome assessment form to summarise the effects of the two regimens.

\section{Additional data collected}

\section{Recurrence of verrucae at 6 months}

Participants were asked whether or not they had a verruca at 6 months on the participant's 6-month questionnaire. If a verruca was present they were asked to record whether or not it was in the original or in a different place. 


\section{Patient satisfaction with treatment}

Patient satisfaction with treatment (on a five-point scale, from 'very unhappy' to 'very happy') was reported on the participant week-1, week-3 and week-12 questionnaires.

\section{Pain associated with first treatment}

Pain associated with the first treatment (on a scale of $0-10$, where 0 is no pain and 10 is the worst pain imaginable) was recorded on the patient pain questionnaire (see Appendix 4). This questionnaire was designed for participants to complete after their first treatment and return to the YTU using a reply-paid envelope.

\section{Pain associated with verrucae and use of painkillers}

Participants were asked to rate how painful their verrucae were on a five-point Likert scale of $0-4$, where 0 was not at all painful and 4 was extremely painful. They were also asked to record if they needed to take a painkiller because of their verruca treatment during the first 3 weeks following entry into the study and, if yes, the number of days they took painkillers. Data were collected on the week-1 and week-3 questionnaires.

\section{Treatment details}

The number of appointments attended by each participant, excluding the week-12 outcome assessment appointment, were recorded by the health-care professional on the podiatrist treatment assessment form. Details of the cryotherapy delivered at each appointment were recorded by the health-care professional on the same form, including the number of freezes performed, the duration of the first freeze on that visit, whether or not the health-care professional considered that sufficient freezing took place and whether or not the patient asked for the freeze(s) to be stopped, and, if so, why. As a means of assessing adherence, the weight of salicylic acid ointment used over the treatment period was recorded by the health-care professional on the podiatrist treatment assessment form by weighing the tubes of salicylic acid at the start and end of treatment. In addition, the number of times salicylic acid was applied within the past 7 days was reported on the participants' week-1 and week-3 questionnaires.

\section{Adverse events}

An adverse event was defined as 'any untoward medical occurrence in a subject to whom a medicinal product has been administered, including occurrences which are not necessarily caused by or related to that product'.

Health-care professionals were asked to report any adverse events occurring in participants in both groups to the trial office using either the 'serious adverse event form' or the 'non-serious adverse event form' (see Appendix 4). The reporting health-care professional was asked to indicate whether or not, in his or her opinion, the event was related to the treatment. Serious adverse events were defined as an event that resulted in death, was life-threatening, required hospitalisation or prolongation of existing hospitalisation, resulted in a persistent or significant disability or incapacity, or resulted in a congenital anomaly or birth defect. When appropriate an assessment of intensity and expectedness was also undertaken.

A list of possible treatment-related adverse events was established, a priori, based on reports in the literature. These were pain, blistering, irritation to the skin, infection, burning sensation, bleeding, scarring and allergic contact reaction.

Health-care professionals were asked to report any serious adverse events within 24 hours of becoming aware of the event and provide a follow-up report if necessary. 


\section{Reasons for stopping treatment and any new treatments}

Whether or not the participant found it necessary to stop the treatment to which they had been allocated and, if so, the reasons for this were recorded on the participant's week-12 questionnaires. Whether or not they started another treatment, and, if so, what was the new treatment, was also recorded on the week-12 questionnaire.

\section{Statistical analysis}

All analyses were conducted on an intention-to-treat basis, including all randomised patients in the groups to which they were randomised. All of the analyses were conducted using STATA statistic and data analysis software version 10.1 (StataCorp LP, College Station, TX, USA), except the logistic regression model accounting for centre clustering effects, which was undertaken using SAS version 9.2 (SAS Institute Inc., Cary, NC, USA) and two-sided significance tests at the $5 \%$ significance level for the primary outcome measure and $1 \%$ significance level for secondary outcome measures. Multiple imputation methods were used to handle missing data. The statistician conducting the analysis remained blind to treatment group and data were unblinded only once all data summaries and analyses were completed.

\section{Trial completion}

The flow of participants through the trial is presented in a CONSORT diagram. The numbers of participants withdrawing from treatment and/or the trial were summarised together with the reasons where available.

\section{Baseline data}

All baseline data were summarised by treatment group and described descriptively. No formal statistical comparisons were undertaken. Continuous measures were reported as means and standard deviations (SDs), whereas categorical data were reported as counts and percentages.

\section{Primary analysis}

The primary outcome was complete clearance of all verrucae at 12 weeks. This was a dichotomous outcome (presence or absence of verruca). We compared the proportions of participants with complete clearance of all verrucae using a chi-squared test.

The Cohen's kappa measure of inter-rater agreement was used to assess the agreement between the two assessors of the blinded photographs whether or not the verrucae had cleared.

\section{Secondary analysis}

\section{Clearance of verrucae at 12 weeks}

A logistic regression model was used to adjust the primary analysis for important prognostic variables (age, whether or not the verrucae have been previously treated and type of verruca). Odds ratios (ORs) and corresponding 95\% confidence intervals (CIs) were obtained from this model.

\section{Time to clearance of verrucae}

Time to clearance was derived as the number of days from randomisation until the date of clearance as detailed from the participant's self-reported questionnaire. Participants' verrucae that had not cleared were treated as censored and their date of trial exit, or date of last available assessment, or 183 days/trial cessation, as appropriate, was used to calculate their duration in the trial. 
A Cox proportional hazards model was used to compare the time to clearance of the verrucae between the two groups, adjusting for the same covariates as for the primary outcome.

\section{Clearance of verrucae at 6 months}

The complete clearance of all verrucae at 6 months was analysed in the same way as the primary outcome, with adjustments for the same covariates.

\section{Number of verrucae at 12 weeks}

Negative binomial regression was used to compare the number of verrucae at 12 weeks between the two treatment groups, with adjustment for the number of verrucae at baseline. These models are used to estimate the number of occurrences of an event when the event has Poisson variation with overdispersion.

\section{Patient's treatment preference}

As patients and health-care professionals were not blinded to treatments, we carried out an analysis to assess the influence of participant's treatment preference on treatment outcomes. A logistic regression model was developed using the primary outcome and included patient preference and an interaction term between randomised treatment and preferred treatment in the model.

\section{Missing data}

We investigated the sensitivity of the results to missing data with multiple imputation analysis. Five imputations were created using a set of appropriate imputation models constructed using variables that were predictive of the missing data. Multiple imputation analysis was performed using the multiple imputation procedure in SAS.

\section{Additional data collected}

The following additional data were collected:

- recurrence of verrucae at 6 months

- patient satisfaction with treatment

- pain associated with the first treatment

- pain associated with verrucae and use of painkillers

- treatment details for the cryotherapy delivered and adherence data for the salicylic acid arm

- adverse events

- reasons for stopping treatment and any new treatments

- if patients had verrucae at 6 months were they in the original or a different place?

All additional data were summarised by treatment group (where appropriate), but no statistical analyses were performed.

\section{Economic analysis}

\section{Aim of the economic analysis}

Economic evaluation of health interventions is a tool used to assist decision-makers in prioritising and allocating resources in the health-care sector, by assessing the value for money (cost-effectiveness) of alternative interventions.

The aim of the economic analysis was to assess the relative costs and effectiveness of cryotherapy and salicylic acid for the treatment of verrucae. Data on both costs and effectiveness of the two comparators were synthesised to assess the additional cost required for an additional unit of 
outcome. For this analysis, a cost-effectiveness approach was taken, where the outcome was defined as complete clearance of verrucae at 12 weeks.

The analysis was conducted on an 'intention-to-treat' basis. Hence, the analysis compared the treatment groups based on their original random allocation, regardless of protocol deviations and participants' compliance or withdrawal. The NHS perspective was taken for the analysis where only costs directly linked to the NHS budget (GP or nurse visits, podiatrist time and cost of equipment and medications) were included.

\section{Data}

\section{Resource use data}

During the participant's treatment period within the study, data on the resource use component of the economic analysis were collected from both participants' self-completed questionnaires and the relevant form (podiatrist treatment assessment form) completed by the health-care professionals.

The number of visits to the podiatrist, nurse or GP for treatment was recorded by the health-care professional who treated the trial participant. In particular, details on the number of cryotherapy sessions administered and the number of tubes of salicylic acid provided to the patients were collected.

In addition, data on other resource usage were collected at 12 weeks after randomisation on a patient self-reported questionnaire. The questionnaire was designed for participant completion and was returned to the trial office using a reply-paid envelope. Participants were asked to complete the questionnaire about the number of visits to the clinic for treatment of their verruca and health service use (e.g. if they had seen a GP, practice nurse or attended an emergency visit with a GP because of their verruca).

\section{Outcome data}

The outcome data used for the economic analysis were the complete clearance of verrucae at 12 weeks. The data on outcome were extracted primarily by two independent assessors from digital photographs taken at 12 weeks. In cases where the digital photograph was not interpretable, the data were extracted from the podiatrist outcome assessment form and, finally, the patient self-reported questionnaire at 12 weeks. This has been described above (see Measurement of primary outcome).

\section{Methods for calculation of costs}

\section{Cost of the cryotherapy treatment}

The cost of cryotherapy treatment comprised two components: the cost of the equipment and the opportunity cost of the health-care professional's time for attending the patients.

The list of equipment required for cryotherapy was compiled by a combination of interviewing podiatrists who run podiatric clinics and the equipment that was bought as part of setting up a trial centre. The equipment list included:

1. cryogenic gloves

2. safety glasses

3. aluminium Dewar

4. tipping trolley for Dewar

5. withdrawal device

6. cryosurgery applicator

7. slim probe

8. apron. 
In the economic analysis, annuitisation of the equipment cost was performed (see Equation 1). For this procedure, the cost of the equipment $(K)$, which was incurred on its purchase, is spread over the lifetime of the equipment to obtain an equivalent annual cost $(E) .{ }^{16}$ An interest rate $(r)$ of $3.5 \%$ and a lifespan $(n)$ for the cryogenic equipment of approximately 5 years were used in the calculations of the annuity factor.

$$
K=E \frac{1-(1+r)^{-n}}{r}
$$

[Equation 1]

To assign an equipment cost per treatment, the annual cost $(E)$ was divided by the maximum number of the treatments that can be provided by a GP or podiatrist. The maximum number of treatments was calculated based on an average appointment time of 20 minutes and assuming full capacity of the clinics for the total number of working days per year (i.e. 253 excluding bank holidays in the UK). The average appointment time of 20 minutes was based on the experience of podiatrists and practice nurses.

In addition to the equipment cost, the cost of liquid nitrogen, which was the freezing agent for the cryotherapy, was calculated. Liquid nitrogen is nitrogen in a liquid state at a very low temperature. ${ }^{17}$ Hence, the Dewars are refilled frequently, approximately every $4-6$ weeks, even though the liquid nitrogen is not being used fully for patient treatments. It is, therefore, difficult to assess the quantity of liquid nitrogen that is required for a single treatment. However, from the trial data, it was noticed that in one trial centre (Galway, Ireland) that exclusively treated trial participants, four refills of a 25-litre Dewar were ordered in a time frame of 3 months. The cost of liquid nitrogen per treatment was calculated by dividing the cost of four refills of a 25-1 Dewar by the total number of treatments performed in that centre.

The clinician's time was calculated based on an average appointment time of 20 minutes. The treatments were administered to the trial participants by either a GP, nurse or podiatrist. The unit costs for these health-care professionals were retrieved from the Unit costs of health and social care 2009. ${ }^{18}$

\section{Cost of the salicylic acid treatment}

The cost of the salicylic acid treatment comprised two components: the cost of the medication and the health professional's time spent for each treatment assessment visit.

The cost of the medication included the:

Verrugon ointment tubes

- felt pads

- plasters.

The cost of Verrugon tubes was calculated based on the number of tubes used by the patients, irrespective of whether or not the patient had used up the entire content of the last tube received. For example, if the patient did not finish the second tube, the total of the two tubes was used for cost calculations. It should be noted that the maximum number of tubes used per participant in the trial was two.

The total numbers of felt pads and plasters were calculated based on the total number of applications, which, in turn, was based on the number of Verrugon tubes that a patient used. Hence, if the patient used only one tube of Verrugon then he or she needed pads and plasters sufficient for 28 applications. This is half of the total number of applications possible during the 8-week treatment period of the trial. Similarly, if the patients used two tubes then pads and plasters were required for 56 applications. 
The total number of felt pads boxes used per patient was calculated by dividing the number of applications by 36 (this is the total number of felt pads in a box: www.nu-careproducts.co.uk/ chiropody.htm\#feltpads, product PPD $126^{19}$ ) and rounded up to a whole number. Similarly, for the plasters, a box of 10 fabric strips sold by a national pharmacy chain,,$^{20}$ equivalent to 20 applications, was used as a reference. The total number of boxes needed for the treatment was calculated by dividing the number of applications by 20 and rounding up to a whole number.

The cost of the health-care professional's time for the administration of treatment was calculated based on an average appointment time of 20 minutes.

\section{Unit costs of the treatments}

The unit costs for the cryotherapy equipment were retrieved either from the supplier's website or from a catalogue that was sent to the different trial centres. When more than one type of the same item was available, the average unit cost was calculated. The unit costs for the cryotherapy equipment are presented in Table 1.

The cost data that were used for calculating the cost of liquid nitrogen per treatment were retrieved from the purchases of liquid nitrogen of a single centre. The costs included the cost of the liquid nitrogen and the cost of delivery. The average cost over four purchases was calculated. Details are provided in Table 2.

For the salicylic acid treatment, the unit costs for the medication, pads and plasters are presented in Table 3.

TABLE 1 Unit cost of cryotherapy equipment

\begin{tabular}{|c|c|c|c|c|c|c|}
\hline Item & Source & Size/type & Price $e^{a, b}$ & $\begin{array}{l}\text { Average } \\
\text { price }\end{array}$ & Price $(£)^{c}$ & $\begin{array}{l}\text { Price including } \\
\text { VAT }(£)^{d}\end{array}$ \\
\hline Cryogenic gloves & $\begin{array}{l}\text { Catalogue sent to } \\
\text { Galway }\end{array}$ & & $€ 35.00$ & $€ 35.00$ & 28.88 & 33.94 \\
\hline Safety glasses & BOC Products ${ }^{21}$ & & $£ 4.42$ & $£ 4.42$ & 4.42 & 5.19 \\
\hline \multirow[t]{2}{*}{ Dewar } & \multirow{2}{*}{$\begin{array}{l}\text { Catalogue sent to } \\
\text { Galway }\end{array}$} & 25 I aluminium & $€ 833.00$ & \multirow[t]{2}{*}{$€ 868.00$} & \multirow[t]{2}{*}{716.30} & \multirow[t]{2}{*}{841.65} \\
\hline & & 25 I stainless steel & $€ 903.00$ & & & \\
\hline $\begin{array}{l}\text { Tipping trolley for } \\
\text { Dewar }\end{array}$ & $\begin{array}{l}\text { Catalogue sent to } \\
\text { Galway }\end{array}$ & & $€ 433.00$ & $€ 433.00$ & 357.33 & 419.86 \\
\hline Withdrawal device & $\begin{array}{l}\text { Catalogue sent to } \\
\text { Galway }\end{array}$ & & $€ 708.00$ & $€ 708.00$ & 584.26 & 686.51 \\
\hline \multirow[t]{2}{*}{ Cryosurgery applicator } & \multirow[t]{2}{*}{$\begin{array}{l}\text { Catalogue sent to } \\
\text { Galway }\end{array}$} & $\begin{array}{l}330 \mathrm{ml} \text { capacity } \\
\text { applicator }\end{array}$ & $€ 630.40$ & \multirow[t]{2}{*}{$€ 642.10$} & \multirow[t]{2}{*}{529.88} & \multirow[t]{2}{*}{622.61} \\
\hline & & $\begin{array}{l}450 \mathrm{ml} \text { capacity } \\
\text { applicator }\end{array}$ & $€ 653.80$ & & & \\
\hline \multirow[t]{3}{*}{ Slim probe } & \multirow{3}{*}{$\begin{array}{l}\text { Catalogue sent to } \\
\text { Galway }\end{array}$} & $1 \mathrm{~mm}$ & $€ 99.40$ & \multirow[t]{3}{*}{$€ 99.40$} & \multirow[t]{3}{*}{82.03} & \multirow[t]{3}{*}{96.38} \\
\hline & & $2 \mathrm{~mm}$ & $€ 99.40$ & & & \\
\hline & & $3 \mathrm{~mm}$ & $€ 99.40$ & & & \\
\hline \multirow[t]{4}{*}{ Cryogenic apron } & \multirow[t]{4}{*}{ BOC Products ${ }^{21}$} & Small & $£ 137.28$ & \multirow[t]{4}{*}{$£ 163.02$} & \multirow[t]{4}{*}{163.02} & \multirow[t]{4}{*}{191.55} \\
\hline & & Medium & $£ 154.44$ & & & \\
\hline & & Large & $£ 171.60$ & & & \\
\hline & & Extra large & $£ 188.76$ & & & \\
\hline
\end{tabular}

a Different prices are provided for different sizes/types of equipment. The average price was calculated.

b All of the prices reported in euros refer to 2009-10 prices.

c Exchange rate: $1 €=0.825232749$ GBP (source: google.co.uk, date 10 June 2010).

d VAT was applied at $17.5 \%$ 
TABLE 2 Cost of liquid nitrogen

\begin{tabular}{|c|c|c|c|c|c|c|}
\hline Item & Source & & Price $(€)^{\mathrm{a}}$ & Average $(€)$ & Price $(£)^{b}$ & $\begin{array}{l}\text { Price including } \\
\text { VAT }(£)^{c}\end{array}$ \\
\hline \multirow{4}{*}{$\begin{array}{l}\text { Liquid nitrogen (calculated } \\
\text { for 25-I Dewar) }\end{array}$} & \multirow{4}{*}{$\begin{array}{l}\text { Galway } \\
\text { invoice }\end{array}$} & Invoice 1 & $2.79 / 1$ & 65.88 & & \\
\hline & & Invoice 2 & $2.79 / 1$ & & & \\
\hline & & Invoice 3 & $2.48 / 1$ & & & \\
\hline & & Invoice 4 & $2.48 / 1$ & & & \\
\hline \multirow[t]{4}{*}{ Delivery charges } & \multirow{4}{*}{$\begin{array}{l}\text { Galway } \\
\text { invoice }\end{array}$} & Invoice 1 & 38/delivery & 27.43 & & \\
\hline & & Invoice 2 & 38/delivery & & & \\
\hline & & Invoice 3 & 16.86/delivery & & & \\
\hline & & Invoice 4 & 16.86/delivery & & & \\
\hline $\begin{array}{l}\text { Total for liquid nitrogen and } \\
\text { delivery }\end{array}$ & & & & 93.31 & 77.00 & 90.47 \\
\hline
\end{tabular}

a All of the prices reported in euros refer to 2009-10 prices.

b Exchange rate: $1 €=0.825232749$ GBP (source: google.co.uk, 10 June 2010).

c VAT was applied at $17.5 \%$.

TABLE 3 Unit costs for the salicylic acid treatment

\begin{tabular}{lll}
\hline Item & Source & Price including VAT (£) $^{\mathrm{a}}$ \\
\hline Verrugon $6 \mathrm{~g}$ & BNF 5922 & 3.00 \\
Fabric plasters & Boots the Chemist & 1.49 \\
Pads & www.nu-careproducts.co.uk/chiropody.htm\#feltpads, product PPD126 & 2.30 \\
\hline
\end{tabular}

BNF, British National Formulary.

a VAT was applied at $17.5 \%$.

\section{Unit costs of the health-care professionals' time}

The unit costs of the health-care professional's time were retrieved from the Unit costs of health and social care $2009^{18}$ document published by the Personal Social Services Research Unit (PSSRU) of Kent University. Unit costs for health-care professionals with the lowest qualifications were chosen. These are presented in Table 4.

\section{Data analysis}

The analysis of data was mainly dictated by the level of missing data for the primary outcome. The base-case analysis was conducted as a 'complete case analysis', where only patients with available primary outcome data were included. Where resource use data were missing, mean values were imputed based on the response group of the patients.

An additional analysis was conducted by including all the patients and performing multiple imputations on both the primary outcome and the missing total costs.

For both analyses, the mean differences in costs and effects and the $95 \%$ CIs around those were calculated by using bias-corrected and -accelerated (BCA) bootstrap methods. For the mean difference in costs, a linear regression was used, whereas logistic regression was used for the difference in primary outcome, given the binary nature of the data.

All the analyses were conducted using STATA statistic and data analysis software, version 10.1. 
TABLE 4 Unit costs for health-care professionals' time

\begin{tabular}{lllll}
\hline Health-care professional & Source & Unit of measurement & Unit cost (£) & Used for \\
\hline Nurse (GP practice) & PSSRU & Per hour (minute) in clinic & $28.00(0.47)$ & Administration of cryotherapy/salicylic acid \\
Nurse (GP practice) & PSSRU & Per surgery consultation & 10 & Additional nurse visits \\
GP & PSSRU & Per surgery/clinic minute & 2.70 & Administration of cryotherapy/salicylic acid \\
GP & PSSRU & Per surgery consultation lasting & 31 & Additional GP visits \\
& & 11.7 minutes & Administration of cryotherapy/salicylic acid \\
$\begin{array}{l}\text { Community chiropodist/ } \\
\text { podiatrist }\end{array}$ & PSSRU & Per clinic visit & 11.00 & \\
\hline
\end{tabular}

\section{Cost-effectiveness analysis}

The cost-effectiveness of cryotherapy versus salicylic acid was assessed by comparing the incremental costs between the two arms of the trial with the incremental benefit, which is expressed as the difference in the proportion of patients with completely cleared verrucae at 12 weeks.

When two options are compared, one is said to 'dominate' the other, and thereby is considered to be the more cost-effective option, if it is associated with a mean cost saving (a negative incremental cost) and positive mean incremental effect. Where one intervention does not dominate the other it is usual practice to calculate the incremental cost-effectiveness ratio (ICER) associated with each intervention group, relative to the next best alternative.

The ICER was calculated by dividing the mean incremental cost $(\Delta \mathrm{C})$ by the mean incremental effect $(\Delta \mathrm{E})(\mathrm{ICER}=\Delta \mathrm{C} / \Delta \mathrm{E})$, where $\mathrm{E}$ is the difference in effectiveness and $\mathrm{C}$ is the cost. Subsequently, the decision-makers can assess whether or not the additional benefit is worth the additional cost. Hence, a treatment strategy can be considered cost-effective only if the decisionmaker's willingness to pay for an additional unit of outcome, i.e. the cost per additional patient cured at 12 weeks, is greater than (or equal to) the ICER. Cost-effectiveness acceptances curves (CEACs) were plotted. CEACs express the probability that a treatment is more cost-effective than its comparator for different thresholds the decision-makers may be willing to pay for an additional unit of outcome. 



\section{Chapter 3}

\section{Protocol changes}

\section{Inclusion and exclusion criteria}

This study was funded via the NIHR HTA's 'medicines for children' call, so the initial inclusion criteria focused on participants aged between 12 and 24 years of age. However, owing to poor recruitment, the possibility of opening up the inclusion criteria to participants over the age of 24 years was considered. The study investigators, TSC and Data Monitoring and Ethics Committee (DMEC) could see no reason why participants over the age of 24 years should not be included in the study. It was felt that including these patients would improve the generalisability of the study's findings, making the results of the study of greater interest to health-care practitioners. Therefore, it was decided to include patients over the age of 24 years, and although there was no known reason why results from participants from older patients should not be applicable to younger patients, it was decided to undertake an analysis looking for an interaction with age.

Following advice from the TSC (20 September 2006 and 19 July 2007), it was decided to exclude the following patients from the study in order to enhance patient safety:

- patients who were currently on renal dialysis

- patients who had cold intolerance, for example Raynaud syndrome or cold urticaria

- patients who had any of the following conditions: blood dyscrasias of unknown origin; cryoglobulinaemia; cryofibrinogenaemia; collagen and autoimmune disease

- patients who were immunosuppressed, for example had agammaglobulinaemia or were currently taking immunosuppressant drugs such as oral corticosteroids

- patients with neuropathy.

\section{Treatment regimens}

In order to increase the generalisability of the study's results it was decided that debridement prior to treatment with cryotherapy was no longer a requirement, but could be performed if it was the site's usual practice. Following advice from the TSC (20 September 2006), further clarifications to the cryotherapy regimen and the treatment of patients with more than one verruca were made (see Chapter 2, Cyrotherapy using liquid nitrogen delivered by the health-care professional).

\section{Clarification of secondary outcomes and analysis}

Following advice from the TSC (20 September 2006) it was decided to clarify the secondary outcomes, the adverse event reporting procedure and the economic analysis plan, and it was decided that the influence of prognostic variables on the primary outcome should be investigated. 


\section{Questionnaire response rates}

The response rate to the 12-week questionnaire was initially lower than anticipated. Results of a systematic review ${ }^{23}$ identified the use of financial incentives as a means of increasing response rates to postal questionnaires. The YTU had also identified that participant questionnaire return rates in previous NIHR HTA trials ${ }^{24}$ could be improved if participants were sent an unconditional $\mathfrak{E 5}$ as a token 'thank you' reimbursement at the end of the trial.

We therefore applied to the regulatory authorities for permission to send participants $\mathfrak{£} 5$ or $€ 5$ with their week-12 questionnaire, i.e. the primary outcome data point. This was not mentioned in the patient information sheet, so that any possibility that it would be interpreted as a financial incentive to taking part in the trial was minimised. The week- 12 questionnaire was preceded by a letter notifying the participant that their week-12 questionnaire was due to arrive shortly. This letter also stated that the questionnaire would be accompanied by a $£ 5$ (or $€ 5$ ) note as a thank you for their taking part in the trial and completing the questionnaires.

\section{Recruitment}

The original proposal contained five recruiting sites that planned to recruit three participants per month over an 18-month period. As the trial progressed, recruitment fell below expected levels despite the recruitment of extra study sites. Details regarding the recruitment of each site can be found in Appendix 1. An extension in time and funding was obtained from the funder and the recruitment period was extended to 39 months (November 2006 to January 2010).

In order to increase the number of eligible patients presenting to the recruiting sites, a variety of recruitment strategies were introduced. Details regarding the recruitment strategies can be found in Appendix 5. 


\section{Chapter 4}

\section{Clinical results section}

\section{Trial recruitment}

Over the course of the trial there was a total of 16 participating sites: 15 in the UK and one in Ireland. These were the podiatry schools at the University of Northampton, the University of Huddersfield, the University of Brighton (at Leaf Hospital, Eastbourne), Glasgow Caledonian University (at Southern General Hospital) and the National University of Ireland, Galway (NUI Galway); Brownlow Group Practice, Liverpool; Springfield Surgery, Bingley; Sheffield PCT podiatry clinic; Sacriston Surgery, Sacriston; The Haven Surgery, Burnhope; Peaseway Medical Centre, Newton Aycliffe; Arlington Road Medical Practice, Eastbourne; Claughton Medical Centre, Birkenhead; Harbinson House Surgery, Sedgefield; Annfield Plain Surgery, Stanley; and Islington PCT podiatry service.

Recruitment of at least one trial participant took place in 14 out of the 16 sites. Recruitment was staggered, with sites joining and leaving the trial over its course. The two sites that did not recruit any patients were Annfield Plain Surgery, because of the short time period between the site initiation visit and the end of the recruitment period, and Islington PCT podiatry services, which withdrew from the study before it had recruited any patients.

Recruitment began in November 2006 and ceased in January 2010. In total, 284 individuals were screened as potential participants and, of these, 242 (85.2\%) were randomised. The overall rate of recruitment is shown in Figure 1. The number of participants recruited per site ranged from 2 to 58 (Table 5). Figure 2 shows the CONSORT flow chart of participants through the trial. Two ineligible participants with hand warts rather than verrucae on their feet were randomised in error, one to each treatment group. These two patients have been excluded from all tables, figures, summaries and analyses (for exceptions, see Table 5 and Figures 1 and 2).

\section{Baseline participant characteristics}

In total, 240 eligible participants were recruited to the study: 117 in the cryotherapy group and 123 in the salicylic acid group. We received a completed baseline questionnaire for 237 participants (114 and 123 individuals in the cryotherapy group and salicylic acid groups, respectively). Three patients did not return their baseline questionnaires. The baseline characteristics are summarised by treatment group in Tables 6 and 7. Data collected on participants' previous verrucae are summarised by treatment group in Table 8 .

The majority of patients in the study were female $(n=157,66 \%)$ and the median age of patients was 24 years, with the youngest person in the study being 12.0 and the oldest person being 75.3 years. The majority ( $n=185,78 \%)$ of participants had received previous treatment for their verrucae. In most cases this included self-treatment using an over-the-counter (OTC) preparation. Preparations previously used included salicylic acid preparations (Bazuka Gel, Bazuka Extra-Strength Gel, Verrugon, Salactol and Boots own-brand gel) and cryotherapy self-treatments (Wartner, Scholl Freeze and Bazuka Sub-Zero). In both groups, a small number 


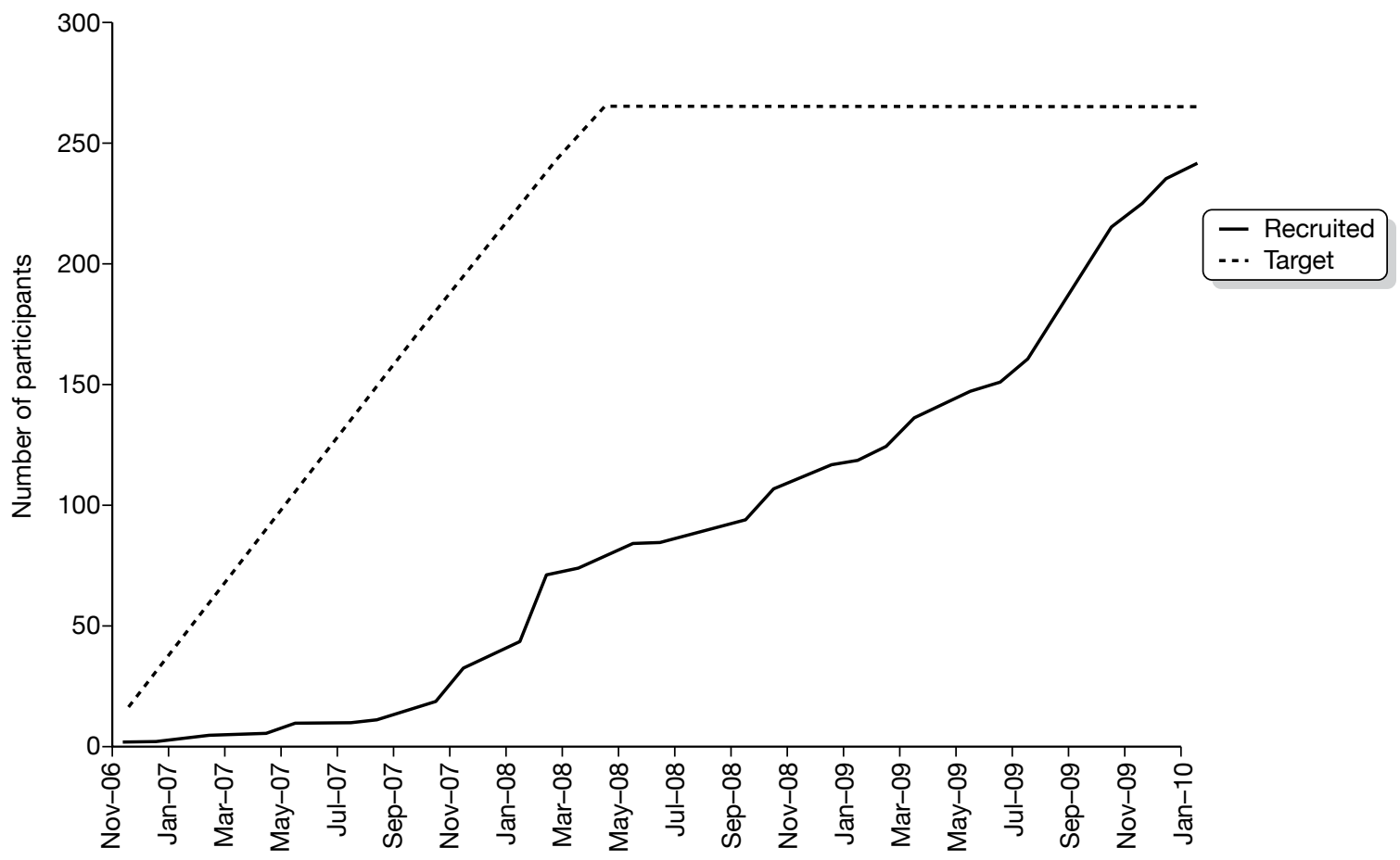

FIGURE 1 Trial recruitment rate.

TABLE 5 Number of participants randomised by group and site

\begin{tabular}{|c|c|c|c|}
\hline & $\begin{array}{l}\text { Cryotherapy } \\
(N=118)\end{array}$ & $\begin{array}{l}\text { Salicylic acid } \\
(N=124)\end{array}$ & $\begin{array}{l}\text { Total } \\
(N=242)\end{array}$ \\
\hline Site & $n(\%)$ & $n(\%)$ & $n(\%)$ \\
\hline University of Northampton & 25 (21.2) & $33(26.6)$ & $58(24.0)$ \\
\hline University of Huddersfield & $24(20.3)$ & $21(16.9)$ & $45(18.6)$ \\
\hline Glasgow Caledonian University & $15(12.7)$ & $21(16.9)$ & $36(14.9)$ \\
\hline Arlington Road Medical Practice & $14(11.9)$ & $5(4.0)$ & $19(7.9)$ \\
\hline Brownlow Group Practice & $8(6.8)$ & $9(7.3)$ & $17(7.0)$ \\
\hline NUl Galway & $5(4.2)$ & $8(6.5)$ & $13(5.4)$ \\
\hline Sacriston Surgery & $6(5.1)$ & $7(5.6)$ & $13(5.4)$ \\
\hline University of Brighton & $6(5.1)$ & $7(5.6)$ & $13(5.4)$ \\
\hline Sheffield PCT & $4(3.4)$ & $5(4.0)$ & $9(3.7)$ \\
\hline Claughton Medical Centre & $3(2.5)$ & $3(2.4)$ & $6(2.5)$ \\
\hline Peaseway Medical Centre & $4(3.4)$ & $1(0.8)$ & $5(2.1)$ \\
\hline Harbinson House Surgery & $2(1.7)$ & $2(1.6)$ & $4(1.7)$ \\
\hline Springfield Surgery & $1(0.8)$ & $1(0.8)$ & $2(0.8)$ \\
\hline The Haven Surgery & $1(0.8)$ & $1(0.8)$ & $2(0.8)$ \\
\hline
\end{tabular}

of individuals (six in the cryotherapy arm and eight in the salicylic acid arm) reported they had tried other treatments, including tea tree oil (four participants).

Just under $60 \%(n=139)$ of participants reported that they were seeking treatment for their verrucae because it was painful; however, when patients were asked how painful their verrucae were, only $34(14 \%)$ reported that they were in quite a lot or extreme pain. A large number of 


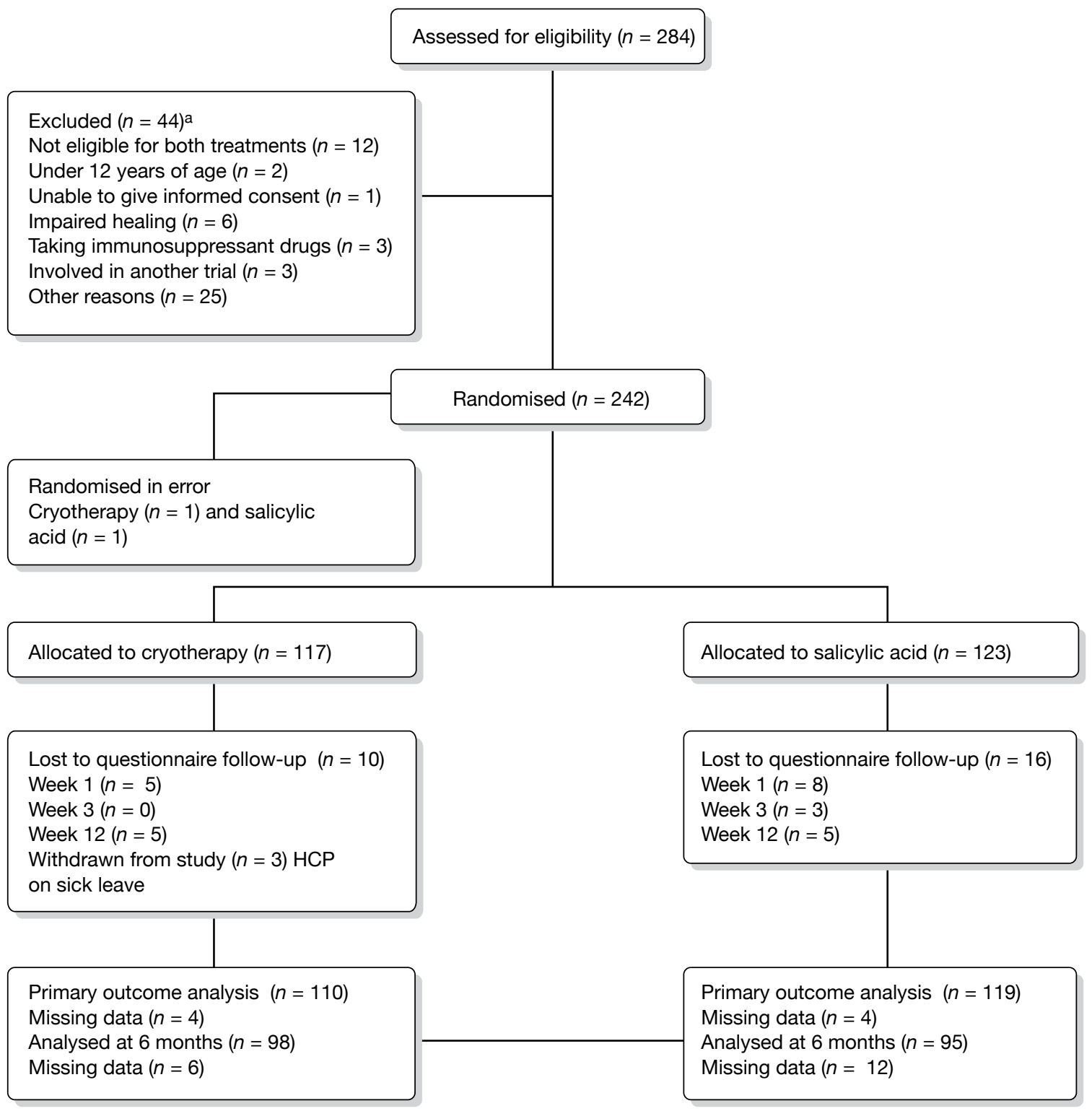

FIGURE 2 EVerT CONSORT diagram. a, More than one category could be checked for each patient. HCP, health-care professional.

TABLE 6 Baseline characteristics

\begin{tabular}{lll}
\hline Characteristics & Cryotherapy $(N=114)$ & Salicylic acid $(\boldsymbol{N = 1 2 3})$ \\
\hline Gender & & \\
Female $n(\%)$ & $84(73.7)$ & $73(59.3)$ \\
Male $n(\%)$ & $30(26.3)$ & $50(40.7)$ \\
Age (years) & & \\
Mean (SD) & $30.1(15.7)$ & $30.2(16.4)$ \\
Median (minimum, maximum) & $24.3(12.2,75.3)$ & $23.2(12.0,70.6)$ \\
\hline
\end{tabular}


TABLE 7 Baseline verrucae characteristics

\begin{tabular}{|c|c|c|}
\hline Characteristics & Cryotherapy & Salicylic acid \\
\hline \multicolumn{3}{|l|}{ No. of verrucae at baseline ${ }^{a}$} \\
\hline N & 106 & 119 \\
\hline Mean (SD) & $4.0(6.6)$ & $3.4(3.6)$ \\
\hline Median (minimum, maximum) & $2.0(1.0,55.0)$ & $2.0(1.0,20.0)$ \\
\hline \multicolumn{3}{|l|}{ Duration of verrucae in months } \\
\hline N & 108 & 119 \\
\hline Mean (SD) & $25.0(24.9)$ & $26.9(24.5)$ \\
\hline Median (minimum, maximum) & $14.5(0.9,144.0)$ & $20(1.5,130.0)$ \\
\hline \multicolumn{3}{|l|}{ Type of verrucae } \\
\hline Mosaic, $n(\%)$ & $29(26.9)$ & $21(17.5)$ \\
\hline Non-mosaic, $n(\%)$ & $79(73.1)$ & $99(82.5)$ \\
\hline \multicolumn{3}{|l|}{ Previous treatment } \\
\hline Yes, $n(\%)$ & $89(78.1)$ & $96(78.0)$ \\
\hline No, $n(\%)$ & $25(21.9)$ & $27(22.0)$ \\
\hline \multicolumn{3}{|l|}{ Type of previous treatment ${ }^{a}$} \\
\hline N & 89 & 96 \\
\hline Self-treatment, $n(\%)$ & $81(91.0)$ & $82(85.4)$ \\
\hline Podiatrist/chiropodist, $n(\%)$ & $24(27.0)$ & $29(30.2)$ \\
\hline GP, $n(\%)$ & $30(33.7)$ & $43(44.8)$ \\
\hline Trial investigating verruca treatments, $n$ (\%) & $0(0.0)$ & $2(2.1)$ \\
\hline Other, $n(\%)$ & $6(6.7)$ & $8(8.3)$ \\
\hline \multicolumn{3}{|l|}{ Reasons for seeking verruca treatment ${ }^{b}$} \\
\hline N & 114 & 123 \\
\hline Painful, $n(\%)$ & $68(59.6)$ & $71(57.7)$ \\
\hline Unable to go swimming, $n$ (\%) & $29(25.4)$ & $41(33.3)$ \\
\hline Unable to participate in other sports, $n(\%)$ & $18(15.8)$ & $28(22.8)$ \\
\hline Other, $n(\%)$ & $46(40.4)$ & $59(48.0)$ \\
\hline \multicolumn{3}{|l|}{ Pain intensity } \\
\hline Not at all, $n(\%)$ & $44(38.9)$ & $44(36.1)$ \\
\hline A little bit, $n(\%)$ & $37(32.7)$ & $34(27.9)$ \\
\hline Moderately, $n(\%)$ & $20(17.7)$ & $22(18.0)$ \\
\hline Quite a lot, $n(\%)$ & $10(8.8)$ & $18(14.8)$ \\
\hline Extremely, $n(\%)$ & $2(1.8)$ & $4(3.3)$ \\
\hline
\end{tabular}

a Data were not received for 15 patients.

b More than one category could be checked so the total for all categories may total more than $100 \%$.

individuals reported 'other' reasons for seeking treatment as a free-text comment. The most frequently reported reasons included the unfavourable appearance of the verrucae, risk of infecting other individuals, the verrucae were annoying or embarrassing, the verrucae had been present for a long time and that the participants just wished to get rid of them. A large number of participants previously had verrucae and the median number of previous verrucae was two, occurring at 5-74 years of age. 
TABLE 8 Previous verrucae characteristics

\begin{tabular}{lll}
\hline Characteristics & Cryotherapy & Salicylic acid \\
\hline Previous verrucae & $72(63.7)$ & \\
Yes, $n(\%)$ & $34(30.1)$ & $81(65.9)$ \\
No, $n(\%)$ & $7(6.2)$ & $36(29.3)$ \\
Don't know, $n(\%)$ & $6(4.9)$ \\
No. of previous verrucae & 69 & \\
$N$ & $3.7(3.5)$ & 76 \\
Mean (SD) & $2.0(1,15)$ & $4.0(4.01)$ \\
Median (minimum, maximum) & $2.0(1,25)$ \\
Age at which previous verrucae occurred (years) & \\
$N$ & 72 & 77 \\
Mean (SD) & $18.9(14.9)$ & $17.9(12.8)$ \\
Median (minimum, maximum) & $13.5(7.0,74.0)$ & $13.0(5.0,60.0)$ \\
\hline
\end{tabular}

In general, the two groups were well balanced at baseline; however, there were slight imbalances in gender and the type of verrucae. The proportion of women was greater in the cryotherapy group than in the salicylic acid group. However, as there is no evidence that gender is a prognostic factor for verrucae clearance, this imbalance is unlikely to affect clearance outcomes and gender was not included in any analyses. The proportion of participants with a mosaic verruca was greater in the cryotherapy group than in the salicylic acid group.

\section{Primary outcome: complete clearance of verrucae at 12 weeks}

In total, 229 participants had a response for whether or not there was complete clearance of all verrucae at 12 weeks after randomisation, with 206 (90.0\%) having a blinded outcome assessment: 159 (69.4\%) had a blinded outcome assessment from a digital photograph with 31 photographs deemed to be of insufficient quality to allow an assessment to be undertaken (The two assessors agreed that they were unable to assess 28 photographs. However, they disagreed on a further 51 photographs. When these 51 photographs were sent to the third assessor she was unable to assess three of these photographs, making a total of 31 photographs which could not be assessed.) Forty-seven (20.5\%) had a blinded outcome assessment from a health-care professional assessment; four (1.7\%) had an unblinded outcome assessment from a health-care professional assessment; and 19 (8.3\%) had patient self-reported data. Overall, 32 of the $229(14.0 \%)$ had complete clearance of all verrucae at 12 weeks: 17 out of the 119 (14.3\%) patients in salicylic acid group and 15 of the 110 (13.6\%) patients in the cryotherapy group. We compared the proportions of participants with complete clearance of all verrucae and there was no evidence of a difference between the salicylic acid and the cryotherapy groups ( $14.3 \%$ vs $13.6 \%$, difference $=0.6 \%, 95 \% \mathrm{CI}$ $-9.6 \%$ to $8.3 \%$; $p=0.89$ ).

\section{Determination of primary outcome}

Table 9 shows the data comparing the outcomes from two independent assessors for photographs from 190 patients. Of these, 106 patients were deemed by both assessors to have verrucae, although both agreed that five patients' verrucae had all cleared. The assessors disagreed in 51 cases: Assessor 1 classified that two patients had no verrucae, whereas Assessor 2 classified these patients as having verrucae. Similarly, Assessor 2 classified that one patient had no verrucae, 
TABLE 9 Results of the blinded classification of clearance of verrucae using photographs by the two independent assessors

\begin{tabular}{llcccr}
\hline & \multicolumn{2}{l}{ Assessor 2 } & & \\
\cline { 2 - 4 } Assessor 1 & Cleared & Not cleared & Unable to assess & Total \\
\hline Cleared & 5 & 1 & 5 & 11 \\
Not cleared & 2 & 106 & 41 & 149 \\
Unable to assess & 0 & 2 & 28 & 30 \\
Total & 7 & 109 & 74 & 190 \\
\hline
\end{tabular}

whereas Assessor 1 classified them as still present. There were five cases in which Assessor 1 classified the verrucae as cleared, but Assessor 2 was unable to assess whether or not the verrucae had cleared. In the remaining 43 cases, the disagreement was between 'not cleared' and 'unable to assess' classifications. To quantify the strength of this association the kappa measure of agreement was estimated as 0.45 [standard error (SE) $0.05,95 \%$ CI 0.35 to 0.55 ]. This indicates a moderate level of agreement.

\section{Secondary outcomes}

\section{Complete clearance of verrucae at 12 weeks adjusted analysis}

The primary analysis was repeated but controlled for age, whether or not the verrucae had been previously treated (yes/no) and the type of verrucae (mosaic/non-mosaic). The results from the logistic regression highlighted that there was no evidence of a difference between the salicylic acid and the cryotherapy groups (OR $0.96,95 \%$ CI 0.44 to $2.11 ; p=0.92$ ).

Age was categorised into three groups ( $<18$ years, $>18$ but $<25$ years, and over 25 years). There was a non-significant effect of age ( $<18$ years vs $>25$ years, OR $0.66,95 \%$ CI 0.22 to 1.98 ; and $>18$ years but $<25$ years vs $>25$ years, OR $0.80,95 \%$ CI 0.35 to 1.82 ).

After adjusting for clustering of healing rates within a centre there was still no evidence of a difference between the salicylic acid and the cryotherapy groups (OR 1.04, 95\% CI 0.43 to 2.50; $p=0.92)$. The resultant intraclass correlation was almost zero $\left(2.74 \times 10^{-10} ; p=1.00\right)$.

\section{Self-reported time to clearance of verrucae}

We compared the time to clearance of the verrucae between the two groups, adjusting for the same covariates as above (age, previous treatment and type of verrucae). There was no evidence of a difference in the time to clearance between the two groups when compared in the Cox proportional hazards model [hazard ratio (HR) $0.80,95 \%$ CI 0.51 to $1.25 ; p=0.33$ ].

\section{Clearance of verrucae at 6 months}

We received data on presence/absence of verrucae at 6 months from 193 participants. Overall, 62 of the 193 (32.1\%) had complete clearance of all verrucae at 6 months: 29 of the 95 (30.5\%) patients in the salicylic acid group and 33 out of the 98 (33.7\%) patients in the cryotherapy group. There was no evidence of a difference between the salicylic acid and the cryotherapy groups ( $30.5 \%$ vs $33.7 \%$, difference $=-3.1 \%, 95 \%$ CI $-10.0 \%$ to $16.3 \%$; $p=0.64)$. The findings from the adjusted analysis were similar to the unadjusted analysis (OR 1.17, 95\% CI 0.62 to $2.21 ; p=0.62$ ). 


\section{Number of verrucae remaining at 12 weeks}

The median number of verrucae at 12 weeks in the salicylic acid group was 2 (minimum to maximum $=0-20$ ) and in the cryotherapy group was 1 (minimum to maximum $=0-40$ ). There was no evidence of a difference in the number of verrucae at 12 weeks between the two groups [incidence rate ratio (IRR) $1.08,95 \%$ CI 0.8 to $1.43 ; p=0.62$ ].

\section{Patient's treatment preference}

Twenty-eight (11.7\%) and 86 (35.8\%) participants expressed a preference at baseline for salicylic acid and cryotherapy, respectively, whereas 104 (43.3\%) did not have a preference and 22 people did not respond to this question. When we extended the primary analysis to include an interaction term between randomised treatment and preferred treatment we found no evidence to suggest that patients' preferences at baseline influenced the outcome.

\section{Missing data}

We investigated the sensitivity of the results to missing data with multiple imputation analysis. There was little difference in the estimates obtained from the complete case analysis and the multiple imputation analysis. The summary of the sensitivity of results to missing data is presented in Table 10.

\section{Patients' willingness to have the same treatment allocation}

There was an association between willingness to have the same allocation and treatment randomised \{chi-squared test statistic 17.90 [2 degrees of freedom (df)]; $p=0.0001$ \}. More patients were willing to have cryotherapy again and fewer patients were willing to have salicylic acid again than expected under independent association.

\section{Additional data collected}

\section{Recurrence of verrucae at 6 months}

Thirty-two patients had clearance of verrucae at 12 weeks. At 6 months, 22 of these 32 patients had reported their verrucae as gone, six had missing data and four (two patients in each group) reported that their verrucae had returned in its original place.

\section{Patient satisfaction with treatment}

Table 11 summarises patient satisfaction with treatment, reported on a five-point scale (from 'very unhappy' to 'very happy') on the participant week-1, week-3 and week-12 questionnaires.

At week 1 , the majority of participants in both groups were happy with their treatment, with 68 individuals (67\%) and 69 individuals (65\%) in the cryotherapy and salicylic acid treatment groups, respectively, answering that they were either 'happy' or 'very happy'. Only a small

TABLE 10 Summary of the sensitivity of results to missing data

\begin{tabular}{lll}
\hline Outcome & Complete cases estimate $\mathbf{( 9 5 \%} \mathbf{~ C l})$ & Multiple imputation estimate $\mathbf{( 9 5 \% ~ C l )}$ \\
\hline Clearance at 12 weeks (unadjusted) & $0.95(0.45$ to 2.00$)$ & 1.01 (0.49 to 2.08) \\
Clearance at 12 weeks (adjusted) & $0.96(0.44$ to 2.11$)$ & $0.99(0.47$ to 2.07$)$ \\
Clearance at 6 months & $1.17(0.62$ to 2.21$)$ & 1.18 (0.68 to 2.08) \\
No. of verrucae & $1.08(0.81$ to 1.43$)$ & $1.06(0.82$ to 1.38$)$ \\
\hline
\end{tabular}


TABLE 11 Patient satisfaction with treatment at weeks 1,3 and 12

\begin{tabular}{|c|c|c|c|c|c|}
\hline \multirow[b]{2}{*}{ Questionnaire } & \multicolumn{5}{|c|}{ Satisfaction with treatment } \\
\hline & Very unhappy & Unhappy & Indifferent & Happy & Very happy \\
\hline \multicolumn{6}{|l|}{ Week 1} \\
\hline Cryotherapy, $n$ (\%) & $6(5.9)$ & $1(1.0)$ & $26(25.7)$ & $43(42.6)$ & $25(24.8)$ \\
\hline Salicylic acid, $n(\%)$ & $1(0.9)$ & $5(4.7)$ & $32(29.9)$ & $54(50.5)$ & $15(14.0)$ \\
\hline \multicolumn{6}{|l|}{ Week 3} \\
\hline Cryotherapy, $n(\%)$ & $10(9.6)$ & $0(0.0)$ & $18(17.3)$ & $48(46.2)$ & $28(26.9)$ \\
\hline Salicylic acid, $n(\%)$ & $3.9(4.0)$ & $8(7.7)$ & $29(27.9)$ & $46(44.2)$ & $17(16.4)$ \\
\hline \multicolumn{6}{|l|}{ Week 12} \\
\hline Cryotherapy, $n$ (\%) & $7(7.9)$ & $5(5.5)$ & $23(25.3)$ & $30(33.0)$ & $26(28.6)$ \\
\hline Salicylic acid, $n(\%)$ & $10(10.2)$ & $21(21.4)$ & 27 (27.6) & $25(25.5)$ & $15(15.3)$ \\
\hline
\end{tabular}

proportion of individuals (7\% in the cryotherapy group and 6\% in the salicylic acid group) were unhappy (answered 'unhappy' or 'very unhappy') with their treatment. At week 3, participants in both groups reported a similar level of satisfaction with their treatment to week 1 . The majority (73\% in the cryotherapy group and $61 \%$ in the salicylic acid group) reported that they were happy, and only $10 \%$ and $12 \%$ in the cryotherapy and salicylic acid treatment groups, respectively, reported that they were unhappy. At week 12 , once again the majority $(62 \%)$ of participants in the cryotherapy group reported that they were happy with their treatment and only $13 \%$ reported that they were unhappy. However, in the salicylic acid group individuals were less happy with their treatment than they were at previous time points and compared with the cryotherapy group at the week-12 time point. Forty individuals (41\%) were happy, whereas 31 individuals (32\%) were unhappy.

Whether or not the participants would be willing to receive the same treatment again is summarised in Table 12.

In total, 146 (77\%) participants indicated at 12 weeks whether they would or would not have the same treatment again. The majority $(n=65,71 \%)$ of cryotherapy patients reported that they would be willing to receive the same treatment again, whereas only 42 (43\%) of salicylic acid patients were willing to repeat their treatment.

\section{Pain associated with first treatment}

Participants were asked to record the level of pain associated with their first treatment. This was reported on a $0-10$ pain scale, where 0 represents no pain and 10 is the worst pain imaginable. The mean pain intensity associated with the first cryotherapy treatment was 3.05 (with a minimum score of 0 and a maximum score of 8 ), whereas the mean pain intensity associated with the first salicylic acid treatment was lower at 0.75 (with a minimum score of 0 and a maximum score of 7).

\section{Pain associated with verrucae and use of painkillers}

Pain associated with participants' verrucae is summarised in Table 13. At weeks 1 and 3 , the majority of participants in both treatment groups reported that their verrucae were not painful or a little painful. A minority reported a lot of pain (answering 'quite a lot of pain' or 'extremely painful'). 
TABLE 12 Participants' willingness to receive allocated treatment again

\begin{tabular}{lll}
\hline Response & Cryotherapy $(\boldsymbol{N}=\mathbf{9 1})$ & Salicylic acid $(\boldsymbol{N}=\mathbf{9 8})$ \\
\hline Yes, $n(\%)$ & $65(71.4)$ & $42(42.9)$ \\
No, $n(\%)$ & $9(9.9)$ & $30(30.6)$ \\
Not sure, $n(\%)$ & $17(18.7)$ & $26(26.5)$ \\
\hline
\end{tabular}

TABLE 13 Pain associated with verrucae

\begin{tabular}{llllll}
\hline & Week $\mathbf{n}$ & & & Week 3 & \\
\cline { 2 - 3 } \cline { 5 - 6 } Verruca painful today? & Cryotherapy & Salicylic acid & & Cryotherapy & Salicylic acid \\
\hline Not at all, $n(\%)$ & $43(41.7)$ & $42(39.3)$ & $62(59.6)$ & $46(44.7)$ \\
A little bit, $n(\%)$ & $35(34.0)$ & $34(31.8)$ & & $25(24.0)$ & $34(33.0)$ \\
Moderately, $n(\%)$ & $16(15.5)$ & $19(17.8)$ & & $13(12.5)$ & $14(13.6)$ \\
Quite a lot, $n(\%)$ & $9(8.7)$ & $10(9.3)$ & & $3(2.9)$ & $8(7.8)$ \\
Extremely, $n(\%)$ & $0(0.0)$ & $2(1.9)$ & & $1(1.0)$ & $1(1.0)$ \\
\hline
\end{tabular}

A minority of participants found it necessary to take painkillers during the first 3 weeks of the study $(n=9)$, with more individuals taking painkillers in the cryotherapy group $(n=8)$ than in the salicylic acid group $(n=1)$. Those individuals who had used painkillers took them for between 1 and 4 days.

\section{Treatment details}

Table 14 summarises the cryotherapy treatment details reported by the treating health-care professional. Out of the 117 patients randomised to cryotherapy, treatment details were returned on the podiatrist treatment assessment form for 109 (93.2\%) individuals and are summarised here. The mean number of visits to the clinic or GP practice for cryotherapy treatment was 3.6, with a minimum of one and a maximum of five visits. The mean duration between visits for treatment was 18.3 days, with a minimum of 9.7 days and a maximum of 52.5 days. At each treatment visit, participants received a mean of 1.6 applications of liquid nitrogen, with each application lasting a mean of 10.9 seconds. In the vast majority of cases (94.2\%), the health-care professional considered that a sufficient freeze had been achieved and for only $9 \%$ of the freezes did the patient request that the freeze was stopped. The main reason for stopping the freeze was that it was painful.

Table 15 summarises the data collected on adherence for the salicylic acid treatment group. The majority (76\%) of individuals received one tube of salicylic acid during the trial, and a mean of $2.8 \mathrm{~g}$ (SD $2.2 \mathrm{~g}$ ) of ointment from each tube was used during the treatment period. Self-reported adherence was reasonably high, with participants applying salicylic acid on a mean of 6.3 days and 5.4 days out of 7 days at weeks 1 and 3, respectively.

\section{Adverse events}

In total, 19 participants reported 28 adverse events. Of these 28 events, one was classed as serious and unrelated to the treatment (salicylic acid). Of the remaining 27 events, 13 were in the salicylic acid group and 14 were in the cryotherapy group. The relationship between the nonserious adverse events and treatment group is reported in Table 16. There were two treatmentrelated non-serious adverse events, both of which were in the cryotherapy group. Both patients developed a blister that was larger in size than expected in routine practice. 
TABLE 14 Cryotherapy treatment details

\begin{tabular}{ll}
\hline Treatment details & Cryotherapy $(\boldsymbol{N}=\mathbf{1 0 9})$ \\
\hline No. of visits & $3.6(0.70)$ \\
Mean (SD) & $4.0(1.0,5.0)$ \\
Median (minimum, maximum) & \\
Duration between visits (days) & \\
Mean (SD) & $18.3(6.8)$ \\
Median (minimum, maximum) & $15.5(9.7,52.5)$ \\
No. of times applied & \\
Mean (SD) & $1.6(0.7)$ \\
Median (minimum, maximum) & $1.5(0.3,4.3)$ \\
Duration of each application (seconds) & \\
Mean (SD) & $9.5(8.6)$ \\
Median (minimum, maximum) & $9.5(2.0,60.0)$ \\
Sufficient freezing took place (\%) & 94.2 \\
Patients asked to stop the freeze (\%) & 9.0 \\
\hline
\end{tabular}

a Two participants had missing data for this variable.

TABLE 15 Salicylic acid treatment details

\begin{tabular}{ll}
\hline Treatment details & Salicylic acid \\
\hline No. of tubes dispensed & 108 \\
$N$ & $82(75.9)$ \\
One tube, $n$ (\%) & $26(24.1)$ \\
Two tubes, $n$ (\%) & \\
Weight of salicylic acid used (g) & 58 \\
$N$ & $2.8(2.2)$ \\
Mean (SD) & $2.4(0.06,9.3)$ \\
Median (minimum, maximum) & \\
No. of times salicylic acid applied in the last 7 days & \\
Week 1 & \\
$N$ & 106 \\
Mean (SD) & $6.3(1.5)$ \\
Median (minimum, maximum) & $7.0(0.0,7.0)$ \\
Week 3 & \\
$N$ & \\
Mean (SD) & 103 \\
Median (minimum, maximum) & $5.4(2.8)$ \\
\hline
\end{tabular}




\section{Reasons for stopping treatment and any new treatments}

Table 17 summarises the number of participants who found it necessary to stop their allocated treatment.

There was a low incidence of participants stopping their original treatment. Twenty-one participants (11.5\%) reported stopping their original treatment. Of these, 16 participants were in the salicylic acid group and five in the cryotherapy group. The reasons for stopping treatment are summarised in Table 18.

Of the participants who reported stopping their original treatment, three (15\%) reported starting another treatment. The cryotherapy patient started salicylic acid treatment and one of the salicylic acid patients continued their treatment with salicylic acid after a temporary stop. The other salicylic acid patient did not state which treatment they started.

TABLE 16 Relationship of the non-serious adverse events by randomised group

\begin{tabular}{lccc}
\hline Relationship & Salicylic acid & Cryotherapy & Total (\%) \\
\hline Unrelated & 9 & 7 & 16 \\
Unlikely to be related & 4 & 5 & 9 \\
Possibly related & 0 & 0 & 0 \\
Probably related & 0 & 0 & 0 \\
Definitely related & 0 & 2 & 2 \\
Unable to assess if related & 0 & 0 & 0 \\
Total & 13 & 14 & 27 \\
\hline
\end{tabular}

TABLE 17 Participants stopping allocated treatment

\begin{tabular}{lcc}
\hline & Cryotherapy & Salicylic acid \\
\hline $\begin{array}{l}\text { Necessary to stop the original treatment? } \\
\text { Yes (\%) }\end{array}$ & $5(5.6)$ & $16(17.0)$ \\
No (\%) & $84(94.3)$ & $78(83.0)$ \\
If yes, was another treatment started? & & \\
Yes (\%) & $1(25.0)$ & $2(12.5)$ \\
No (\%) & $3(75.0)$ & $14(87.5)$ \\
\hline
\end{tabular}

TABLE 18 Reasons for stopping treatment

\begin{tabular}{lll}
\hline Reason & Cryotherapy group & Salicylic acid group \\
\hline Pain & 2 & 7 \\
Poor condition of the participant's skin & 0 & 5 \\
Blistering & 2 & 0 \\
Ran out of plasters & 0 & 1 \\
Participant believed verruca had gone & 0 & $4^{\mathrm{a}}$ \\
\hline
\end{tabular}

a Participant started treatment again as found verruca had not gone. 



\section{Chapter 5}

\section{Economic analysis}

This chapter presents the results of the economic analysis of the EVerT trial. We have undertaken several analyses to assess whether or not costing assumptions and missing data could have affected the results.

\section{Summary of the resource usage}

Data on resource usage were collected for the treatment visits, any additional contact with GPs or nurses, and emergency visits to the GP, as well as items related to the medication for both groups. The average resource usage on the EVerT study is reported in Table 19.

During the trial, participants in the cryotherapy arm had a mean of 3.59 visits to the GP, nurse or podiatrist for treatment. The salicylic acid arm participants had a mean of 1.94 visits. Only a small number of patients (three in each group) had extra visits to the GP, in addition to the planned treatment visits. Participants in the cryotherapy arm had a mean of 0.04 additional visits to the GP, whereas those in the salicylic acid arm had a mean of 0.01 additional visits. Eight patients from both groups had additional visits to a nurse. This resulted in a mean number of additional nurse visits of 0.05 for the patients undergoing cryotherapy and 0.08 for the salicylic acid group. Salicylic acid patients received a mean of 1.25 tubes of Verrugon, whereas cryotherapy patients received a mean of 3.49 treatments.

Two emergency visits were reported, one in each group. For the salicylic acid patient, the comments referred to an event that happened before the randomisation date. The cryotherapy patient did see the GP, but, after reviewing the trial co-ordinator's notes for this patient, it was concluded that this visit was already reported as an additional visit to the GP.

\section{Missing data on resource use and outcome}

There was a significant level of missing data on resource usage relating to additional GP or nurse visits: $30 \%$ and $28 \%$ for the cryotherapy and salicylic acid groups, respectively. The level of missing data for treatment visits, number of tubes of Verrugon and cryotherapy applications was much lower, ranging from $2 \%$ to $7 \%$.

The missing items were a result of either the trial participants not returning the questionnaire or not completing the relevant questions on the questionnaire. Missing data on number of treatment visits was because of missing podiatrist treatment assessment forms. The level of missing data was not related to the treatment allocation as demonstrated by a chi-squared test.

Table 20 presents details on the missing data for various resource usage items.

Table 20 supports the notion that the amount of missing data is not related to group allocation, which reduces the risk of bias. In the following analyses we adjusted for missing data through multiple imputation methods. 
TABLE 19 Average resource usage

\begin{tabular}{|c|c|c|}
\hline Item & Cryotherapy & Salicylic acid \\
\hline \multicolumn{3}{|l|}{ Average no. of treatment visits } \\
\hline$n$ & 109 & 120 \\
\hline Mean (SE) & $3.59(0.072)$ & $1.94(0.38)$ \\
\hline SD & 0.75 & 0.42 \\
\hline Median (minimum, maximum) & $4(1,5)$ & $2(1,4)$ \\
\hline Missing (\%) & $8(7 \%)$ & $3(2 \%)$ \\
\hline \multicolumn{3}{|c|}{ Average no. of additional GP visits } \\
\hline$n$ & 82 & 89 \\
\hline Mean (SE) & $0.04(0.03)$ & $0.01(0.01)$ \\
\hline SD & 0.25 & 0.11 \\
\hline Median (minimum, maximum) & $0(0,2)$ & $0(0,1)$ \\
\hline Missing (\%) & $35(30 \%)$ & $34(28 \%)$ \\
\hline \multicolumn{3}{|c|}{ Average no. of additional nurse visits } \\
\hline$n$ & 82 & 89 \\
\hline Mean (SE) & $0.05(0.03)$ & $0.08(0.04)$ \\
\hline SD & 0.27 & 0.34 \\
\hline Median (minimum, maximum) & $0(0,2)$ & $0(0,2)$ \\
\hline Missing (\%) & $35(30 \%)$ & $34(28 \%)$ \\
\hline \multicolumn{3}{|l|}{ Average no. of tubes of Verrugon } \\
\hline$n$ & NA & 116 \\
\hline Mean (SE) & NA & $1.25(0.04)$ \\
\hline SD & NA & 0.44 \\
\hline Median (minimum, maximum) & NA & $1(1,2)$ \\
\hline Missing (\%) & NA & $7(6 \%)$ \\
\hline \multicolumn{3}{|c|}{ Average no. of cryotherapy treatments given to patients } \\
\hline$n$ & 109 & NA \\
\hline Mean (SE) & $3.49(0.08)$ & NA \\
\hline SD & 0.80 & NA \\
\hline Median (minimum, maximum) & $4(1,5)$ & NA \\
\hline Missing (\%) & $8(7 \%)$ & NA \\
\hline
\end{tabular}

NA, not applicable.

\section{Data analysis}

Given the level of missing data for both the primary outcome and the resource use items, the analysis of data was based on two scenarios as will be described below.

\section{Scenario 1: complete case analysis based on the primary outcome data}

For the first scenario, only the patients who had primary outcome data were considered. Mean values were imputed for the missing resource usage items (i.e. treatments visits, additional visits to the GP or nurse, number of cryotherapy treatments, number of tubes of Verrugon). For the visits, the mean imputation was performed based on the outcome group (i.e. verrucae gone or not gone) and the treatment allocation. For the cryotherapy treatments and the number of Verrugon tubes, the means were imputed based on the outcome group only. 
TABLE 20 Missing data on resource use items and outcomes

\begin{tabular}{|c|c|c|c|}
\hline \multirow[b]{2}{*}{ Resource use or outcome data item } & \multicolumn{2}{|l|}{ Missing response, $n(\%)$} & \multirow{2}{*}{$\begin{array}{l}\text { Treatment arm impact on level of } \\
\text { missing data (Pearson chi-squared, } \\
p \text {-value) }\end{array}$} \\
\hline & Cryotherapy $(n=117)$ & $\begin{array}{l}\text { Salicylic acid } \\
(n=123)\end{array}$ & \\
\hline Additional visits to GP or nurse & $35(30)$ & $34(28)$ & $(0.1511, p=0.697)$ \\
\hline Treatment visits & $8(7)$ & $3(2)$ & $(2.6528, p=0.103)$ \\
\hline No. of tubes of Verrugon & NA & $7(6)$ & NA \\
\hline No. of cryotherapy treatments & $8(7)$ & NA & NA \\
\hline Primary outcome & $7(6)$ & $4(3)$ & $(0.1345, p=0.714)$ \\
\hline
\end{tabular}

NA, not applicable.

The total costs were calculated by summing up the cost of treatment visits, additional GP or nurse visits and the medication costs, i.e. the cost of cryotherapy equipment and liquid nitrogen per patient treatment for the cryotherapy group and the cost of Verrugon, pads and plasters for the salicylic acid group. Table 21 presents the costs by items of resource usage. The majority of costs for both groups was owing to the cost of treatment visits, with the average cost per patient being larger in the cryotherapy group than in the salicylic acid group ( $£ 88.69 \mathrm{vs} £ 39.59$ ). The second largest cost for the cryotherapy group was the cost of treatment, which included the cost of equipment and liquid nitrogen. The average cost of the cryotherapy treatment per patient was $£ 60.05$.

To avoid any distributional assumptions on the cost and outcome data, the BCA 95\% CIs around the mean difference in costs and outcomes were calculated by the bootstrapping method. The mean differences in costs and the proportion of patients with cleared verrucae were calculated based on linear regression for the former and logistic regression for the latter. Two types of analyses were conducted: first, based on unadjusted costs and outcomes and, second, by adjusting them based on the age of the participants, whether or not they had received previous treatment and the type of verrucae.

\section{Unadjusted costs and outcomes}

The results of the base case unadjusted analysis demonstrate that there is a significant difference in the total cost per patient between the two arms of the study, with cryotherapy costing on average $£ 101.17$ more per patient. The treatment effect for cryotherapy is smaller than that for salicylic acid, although statistically non-significant. The mean total costs and outcomes based on data after imputation are presented in Table 22, whereas the mean difference in costs and outcomes and the corresponding 95\% CI are presented in Table 23.

\section{Adjusted costs and outcomes}

The adjusted results lead to the same conclusion as the unadjusted results, i.e. cryotherapy is more costly and less effective (not statistically significant) than salicylic acid treatment. Table 24 presents the mean difference in adjusted costs and outcomes.

\section{Cost-effectiveness and uncertainty}

As demonstrated in Tables 23 and 24, cryotherapy is more costly and non-significantly less effective. The bootstrapped pairs of difference in costs and outcomes for both adjusted and unadjusted results are presented on a cost-effectiveness plane (CE plane) (see Figure 3) to visually demonstrate the above conclusions. As evident from the CE plane, almost $50 \%$ of the bootstrapped replicates falls either side of the line that goes through the zero difference 
TABLE 21 Costs by item of resource usage

\begin{tabular}{llcl}
\hline Item & Treatment group & Mean cost (£) & $\mathbf{9 5 \%} \mathbf{C l}$ \\
\hline Treatment visits (health-care & Salicylic acid & 39.59 & 33.26 to 45.92 \\
professional's time) & Cryotherapy & 88.69 & 74.70 to 102.68 \\
Verrugon (including pads and plasters) & Salicylic acid & 8.50 & 7.97 to 9.03 \\
Cryotherapy cost (liquid nitrogen and & Cryotherapy & 60.05 & 57.57 to 62.53 \\
equipment cost) & & & \\
Additional visit to GP & Salicylic acid & 0.35 & -0.17 to 0.86 \\
& Cryotherapy & 1.15 & -0.09 to 2.38 \\
Additional visit to nurse & Salicylic acid & 0.78 & 0.24 to 1.31 \\
& Cryotherapy & 0.49 & 0.06 to 0.93 \\
Total costs & Salicylic acid & 49.22 & 42.39 to 56.04 \\
& Cryotherapy & 150.39 & 135.65 to 165.13 \\
\hline
\end{tabular}

TABLE 22 Mean total costs and outcomes based on data after imputation

\begin{tabular}{lllll}
\hline Variable & Treatment & $\boldsymbol{n}$ & Mean cost/outcome & SE \\
\hline Costs $(£)$ & Salicylic acid & 119 & 49.22 & 3.46 \\
& Cryotherapy & 110 & 150.39 & 7.48 \\
& Difference $=101.17 ; p<0.001$ & & \\
Outcomes & Salicylic acid & 119 & 0.143 (17 patients) & 0.032 \\
& Cryotherapy & 110 & 0.136 (15 patients) & 0.033 \\
& Difference $=-0.006 ; p=0.89$ & & \\
& & & & \\
& & & &
\end{tabular}

TABLE 23 Mean difference in costs and outcomes (BCA 95\% Cl)

\begin{tabular}{llll}
\hline Variable & Mean difference & $95 \% \mathrm{Cl}$ & ICER \\
\hline Costs $(£)$ & 101.17 & 85.09 to 117.26 & Cryotherapy is dominated \\
Outcomes & -0.0065 & -0.10 to 0.08 & \\
\hline
\end{tabular}

in outcomes point ( $x$-axis). This is indicative of high uncertainty around the difference in effectiveness of the two treatments. In contrast, all of the cost replicates are above the zero line of the $y$-axis, i.e. no difference in costs. Figure 3 presents the CE plane for both unadjusted and adjusted results.

Figure 4 presents the CEACs. This demonstrates the probability of the cryotherapy being costeffective given a specific willingness-to-pay value per 'cured' patient. The adjusted and unadjusted data give the same results. In both cases, the probability that cryotherapy is cost-effective is around $40 \%$ at a threshold value of $£ 15,000$ per cured patient.

\section{Sensitivity analysis based on the data analysis of scenario 1 (complete case for primary outcome data)}

The majority of costs for the cryotherapy group are owing to treatment visits (i.e. health-care professional's time) and the cost of the treatment itself, i.e. the cost of the equipment and liquid nitrogen. A sensitivity analysis was carried out by adopting an extreme approach whereby the administration of the treatment was assumed to be carried out by a nurse (rather than a GP) in those study sites that were set up in GP practices and by excluding completely the cost of cryotherapy equipment and liquid nitrogen. In effect, this analysis would result in comparing 
TABLE 24 Mean difference in adjusted costs and outcomes (BCA 95\% Cl)

\begin{tabular}{llll}
\hline Variable & Mean difference & $95 \%$ Cl & ICER \\
\hline Costs $(£)$ & 101.21 & 84.18 to 118.25 & Cryotherapy is dominated \\
Outcomes & -0.00336 & -0.09 to 0.08 & \\
\hline
\end{tabular}
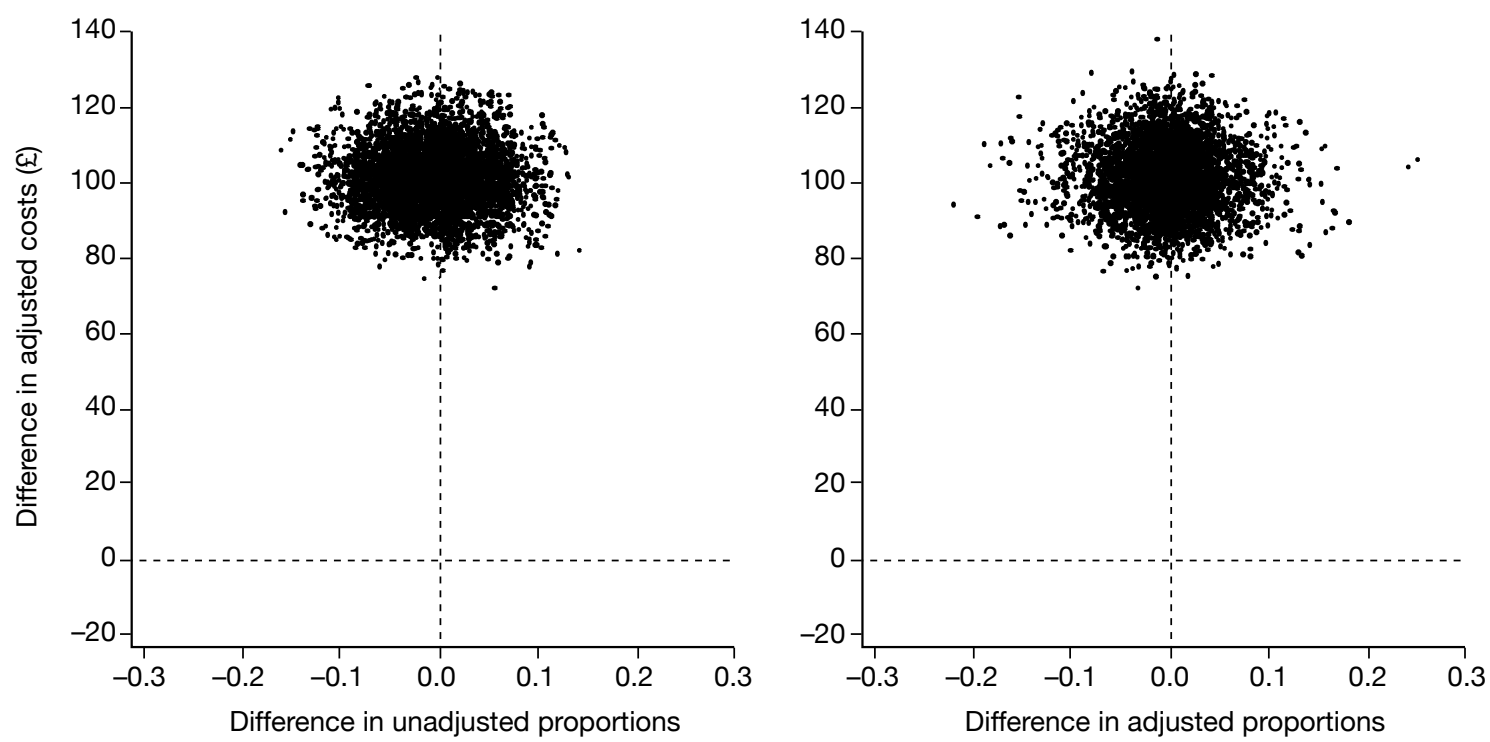

FIGURE 3 Cost-effectiveness plane for unadjusted and adjusted costs and outcomes.

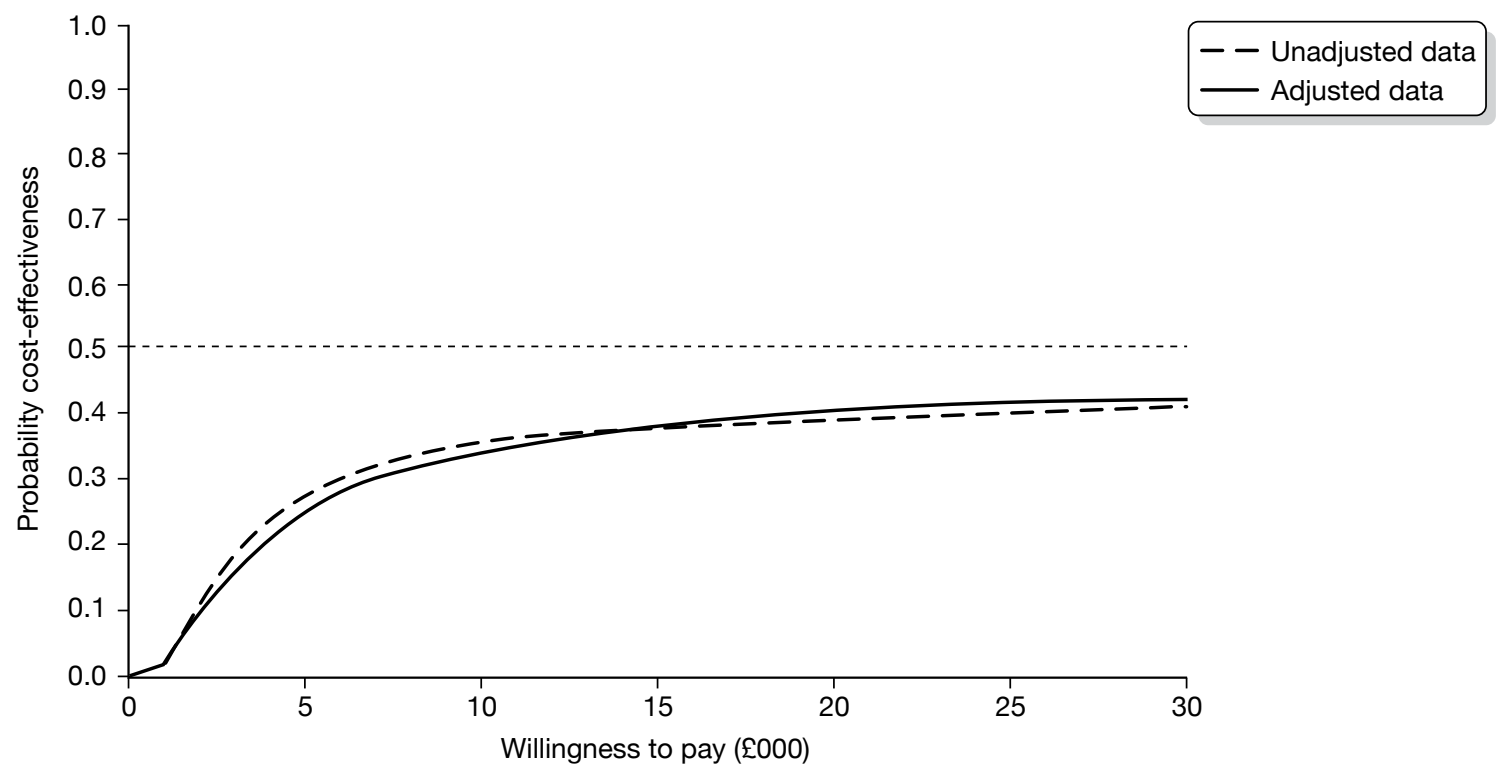

FIGURE 4 Cost-effectiveness acceptability curves (adjusted and unadjusted data).

both treatments based on the treatment visits only rather than including the cost of medication as well. Table 25 presents the results of the sensitivity analysis. Cryotherapy is again more costly than salicylic acid treatment. This is because of the greater number of treatment visits that the patients made, even though the cost of the health-care professional who administered the cryotherapy was lower (nurse vs GP). 
TABLE 25 Difference in cost and outcomes

\begin{tabular}{llll}
\hline Variable & Mean difference & $95 \% \mathrm{Cl}$ & ICER \\
\hline Unadjusted & & & \\
Costs $(£)$ & 9.18 & 7.09 to 11.26 & Cryotherapy is dominated \\
Outcome & -0.0065 & -0.10 to 0.08 & \\
Adjusted & & & \\
Costs $(£)$ & 9.17 & 7.00 to 11.33 & Cryotherapy is dominated \\
Outcomes & -0.00336 & -0.09 to 0.08 & \\
\hline
\end{tabular}

Figures 5 and 6 present the CE planes and CEACs for both unadjusted and adjusted results of the sensitivity analysis. Cryotherapy remains more costly and all of the bootstrapped replicates of difference in costs are above the zero line, although outcome results do not change. The smaller difference in costs between the two treatments (compared with the base-case analysis) results in cryotherapy having an approximately $40 \%$ probability of being cost-effective at a lower (than the base-case analysis) threshold.

\section{Scenario 2: multiple imputation on the primary outcome data and on the missing total costs}

Data were imputed by using multiple imputation methods for the 11 patients who had missing primary outcome data. The multiple imputations were performed by using age, previous treatment and type of verrucae as covariates. Data were imputed also for the missing total costs of these 11 patients.

The mean differences in costs and outcomes after multiple imputation are presented in Table 26. The CE plane and CEAC are presented in Figures 7 and 8. The results of the multiple imputation do not alter the overall conclusion of the study that cryotherapy is more costly than salicylic acid and that there is no evidence of it being more effective.

\section{Summary of findings}

The EVerT trial has demonstrated that there is no evidence of a difference in effectiveness between cryotherapy and salicylic acid at 12 weeks. In fact, cryotherapy appears to be marginally worse than salicylic acid, without reaching statistical significance. Cryotherapy is also more expensive than salicylic acid, at an average incremental cost of approximately $£ 101$ per patient. This evidence results in cryotherapy being dominated (i.e. more costly, less effective) by salicylic acid.

Two scenarios for analysing the data were developed. One was based on complete case analysis for patients who had primary outcome data and mean imputation of cost data for the patients with missing information on different cost items. The second analysis was based on multiple imputation of the primary outcome and missing total cost data. Both analyses resulted in the same conclusions, i.e. cryotherapy is more costly and less effective than salicylic acid, and, hence, dominated by salicylic acid.

An extreme case sensitivity analysis was conducted by replacing the provision of treatment from a GP with nurses, and excluding the cost of cryotherapy equipment and liquid nitrogen, the 

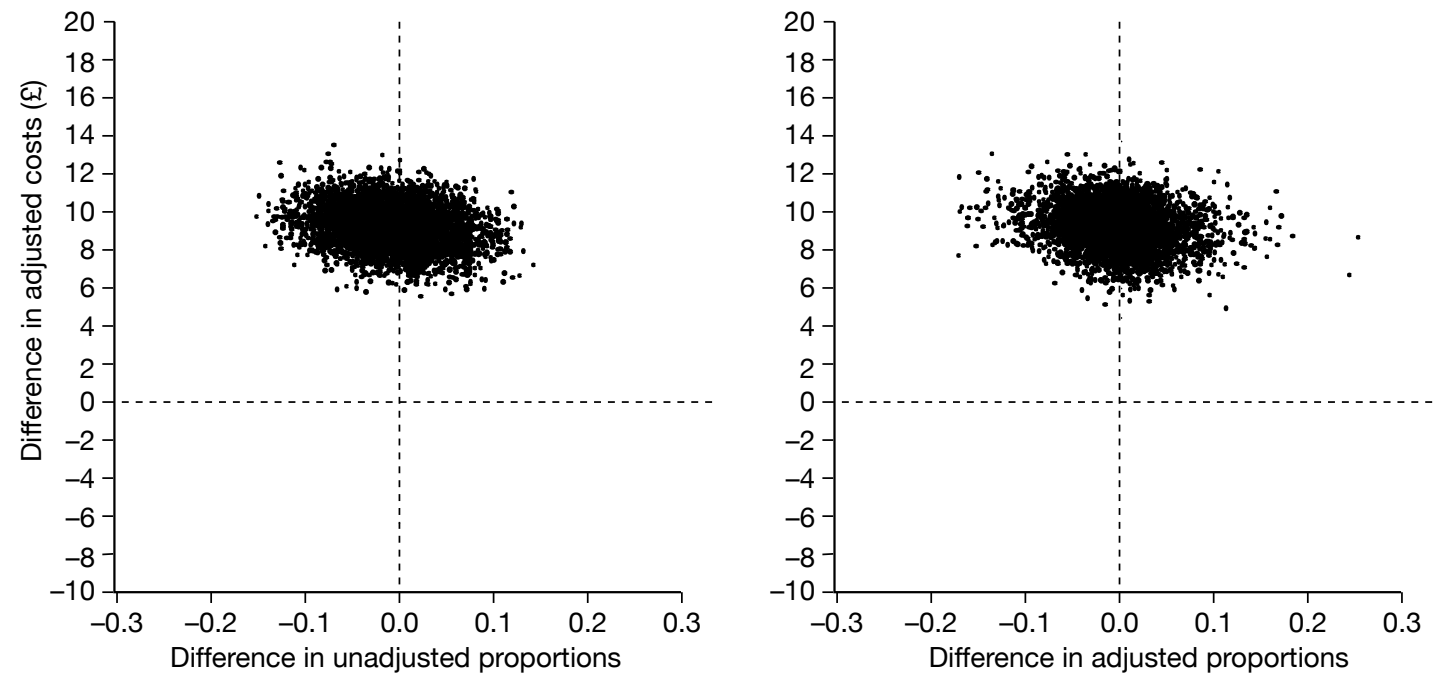

FIGURE 5 Cost-effectiveness planes for unadjusted and adjusted costs and outcomes.

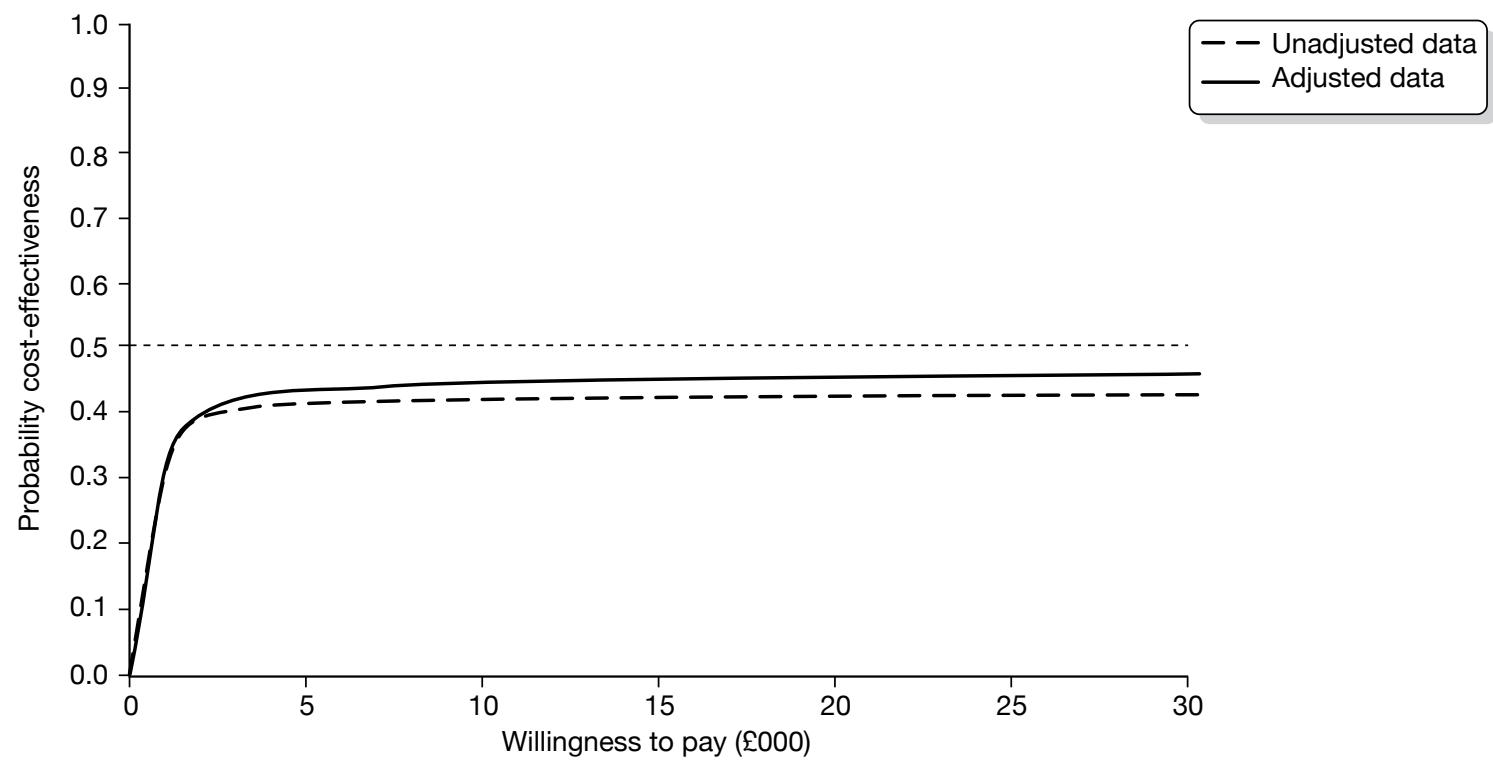

FIGURE 6 Cost-effectiveness acceptability curve for the sensitivity analysis.

TABLE 26 Mean difference in costs and outcomes after multiple imputation

\begin{tabular}{llll}
\hline Variable & Mean difference & $\mathbf{9 5 \%}$ Cl & ICER \\
\hline Costs $(£)$ & 101.39 & 86.29 to 117.29 & Cryotherapy is dominated \\
Outcomes & -0.0083 & -0.10 to 0.08 & \\
\hline
\end{tabular}

implicit assumption being that the equipment has a dual use. However, this analysis still resulted in cryotherapy being more expensive than salicylic acid. By excluding the cryotherapy treatment costs completely and reducing the cost of the health-care professional who administers the treatment it is made evident that the results are strongly driven by the lack of effectiveness as well as the larger number of treatment visits that cryotherapy patients have. When the costs of the 


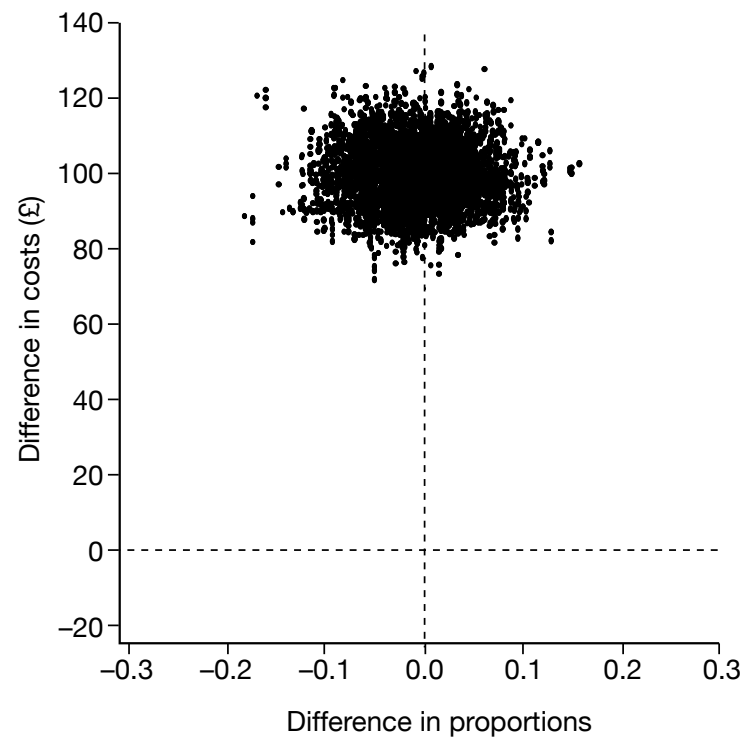

FIGURE 7 Cost-effectiveness plane (multiply imputed data).

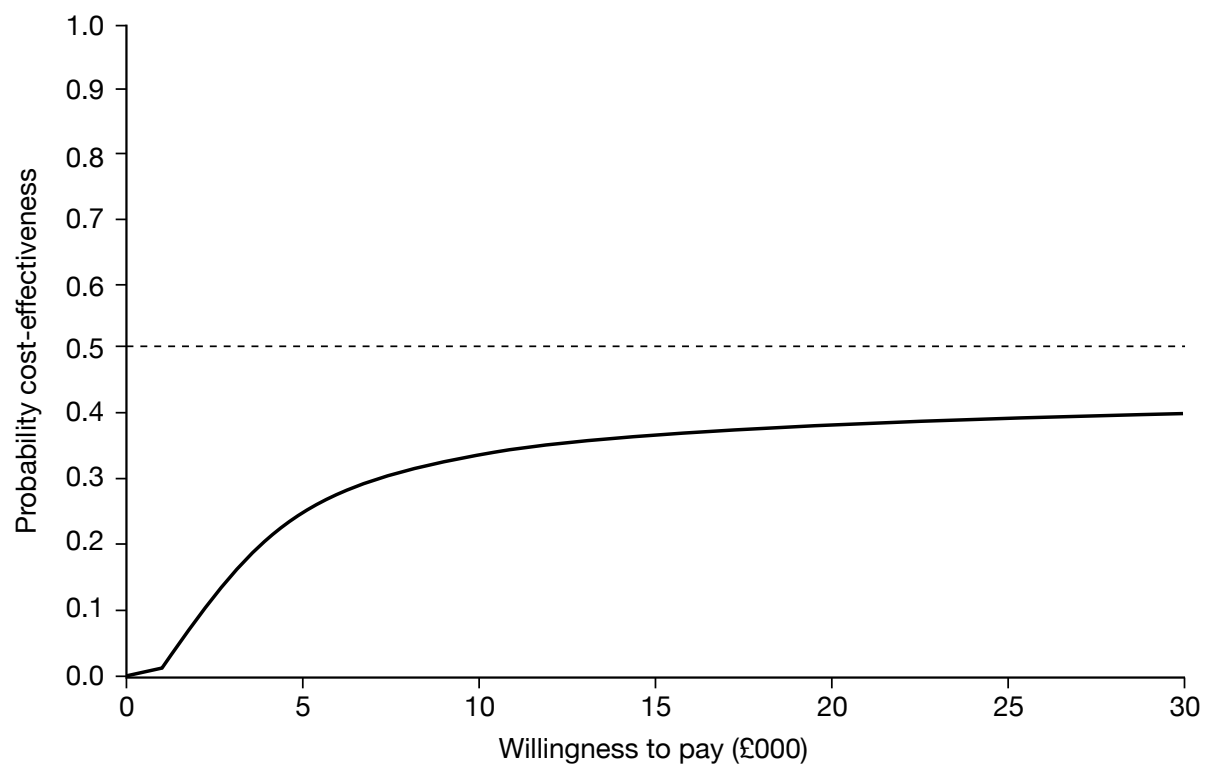

FIGURE 8 Cost-effectiveness acceptability curve (multiply imputed data).

cryotherapy equipment and liquid nitrogen are included, it is found that cryotherapy is even less cost-effective.

In conclusion, self-treatment with salicylic acid is more cost-effective for the treatment of verrucae than cryotherapy administered by a health-care professional. 


\section{Chapter 6}

\section{Discussion}

Tere we report the results of a large RCT assessing the clinical effectiveness and costeffectiveness of cryotherapy and salicylic acid for the treatment of verrucae. We were motivated to conduct this trial when the Cochrane systematic review into the treatment of cutaneous warts highlighted the lack of good-quality evidence to support the use of cryotherapy over simple topical treatments. This discussion summarises the key findings, compares these results with published studies, considers the strengths and limitations of the present study and summarises the clinical and research implications of the work.

\section{Key findings}

We compared the clinical effectiveness and cost-effectiveness of cryotherapy using liquid nitrogen and $50 \%$ salicylic acid for the treatment of verrucae and found no evidence to suggest that cryotherapy was more effective than salicylic acid in clearing verrucae. Overall, 32 of 229 (14.0\%) patients had complete clearance of all verrucae at 12 weeks; 17 out of $119(14.3 \%)$ were in the salicylic acid group and 15 of $110(13.6 \%)$ in the cryotherapy group, $p=0.89$. When the analysis was repeated, adjusting for potentially important prognostic variables (age, whether or not the verrucae had previously been treated and type of verruca), there was no difference in the overall findings (OR 0.96, 95\% CI 0.44 to $2.11 ; p=0.92$ ). In addition, cryotherapy is associated with higher costs per cured patient. The combination of lack of difference in effectiveness between cryotherapy and salicylic acid, and higher costs leads to cryotherapy being dominated by salicylic acid. Sensitivity analysis conducted by completely excluding the cost of liquid nitrogen and the cost of cryotherapy equipment, and assuming that the provision of the treatment is undertaken by a nurse instead of a GP, did not alter the conclusions of the study, i.e. there is very small probability of cryotherapy being cost-effective for a wide range of willingness-to-pay values. The sensitivity analysis clearly demonstrates, primarily, the lack of treatment benefit of cryotherapy over salicylic acid and, secondly, that the larger number of treatment visits required for the cryotherapy drives the cost-effectiveness results.

\section{Comparison with other studies/reviews}

Our results confirm the findings of the two published studies ${ }^{25,26}$ and the results from a more recent Dutch primary care study, ${ }^{15}$ which compared cryotherapy with a salicylic acid or a combination of salicylic and lactic acid for the treatment of plantar and hand warts. These previous studies, like ours, showed no evidence for the effectiveness of cryotherapy compared with salicylic acid alone or in combination with lactic acid for the treatment of plantar warts. In Figure 9 we put the two studies reporting clearance rates for plantar warts ${ }^{15,26}$ (including our own) in a meta-analysis (the results of the Bunney et al. trial ${ }^{25}$ have not been included, as this trial included only participants with hand warts), which shows that the OR for cure is 1.07 (95\% CI 0.63 to 1.79 ). This result is not statistically significant and indicates that the odds of clearance of verrucae was similar in both groups. 


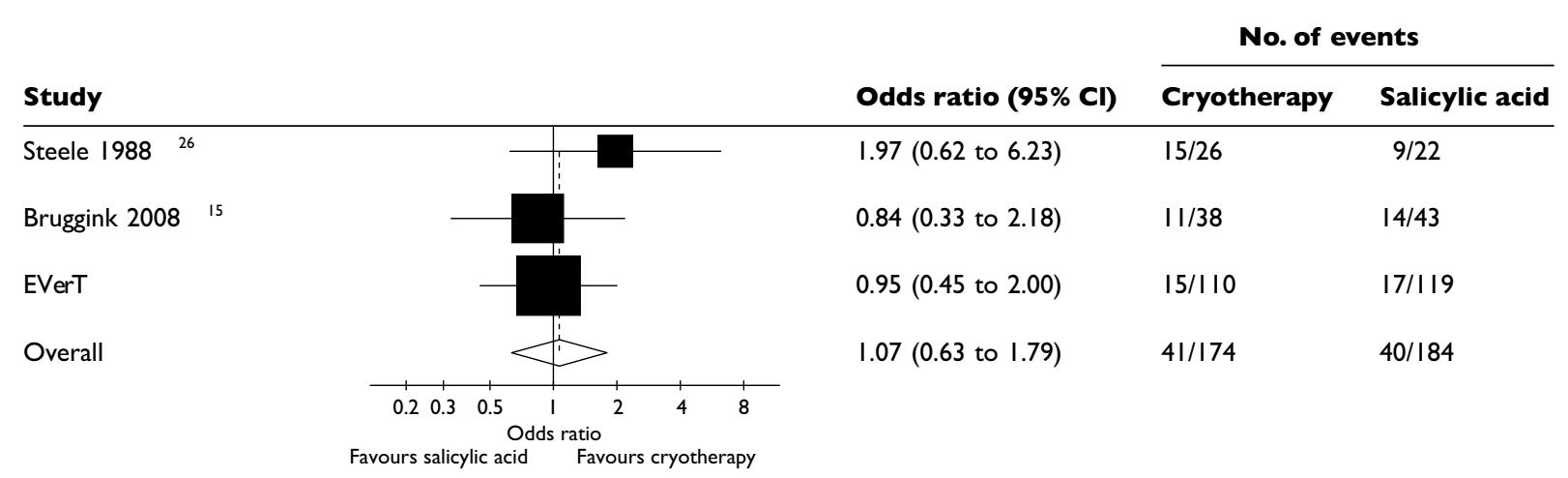

FIGURE 9 Forest plot of RCTs comparing salicylic acid and cryotherapy for the treatment of verrucae.

Our trial, however, does differ from the previous studies with respect to the cure rate. The cure rate in previous studies ranged from about $30 \%{ }^{15}$ to $68 \%,{ }^{26}$ which is at least twice the cure rate we observed.

This difference in cure rate could be attributed to different populations recruited to the study. The type of wart being treated was different between the studies. For example, Bunney and colleagues ${ }^{25}$ included only patients with hand warts, whereas Steele and $\operatorname{Irwin}^{26}$ excluded what are generally regarded as harder-to-treat mosaic warts. They also excluded patients with five or more lesions, lesions outside an average diameter of $3-9 \mathrm{~mm}$ and patients who had self-treated within the past month. In our study, $22 \%$ of participants had a mosaic wart, $17 \%$ had more than five verrucae and patients were not excluded if they had tried previous treatment. There was a difference in the age of the populations. Patients were younger in the Steele and $\operatorname{Irwin}^{25}$ and Bruggink et al. ${ }^{15}$ studies, with $59 \%$ of participants under the age of 16 in the Steele and Irwin study ${ }^{25}$ compared with $17.3 \%$ in our study. The median age of patients in the Bruggink et al. study ${ }^{15}$ was 15 [interquartile range (IQR) 7-39] for cryotherapy patients and 13 (IQR 7-31) years for salicylic acid patients compared with median ages of 24 years and 23 years, respectively, in our study.

\section{Treatment regimen}

We anticipated that a large percentage of potential participants would have received some form of treatment of their verruca prior to entry into the trial. In the UK the first line of treatment is generally an OTC salicylic acid preparation with a strength of $15-26 \%$ salicylic acid, with cryotherapy treatment using liquid nitrogen and higher concentrations of salicylic acid viewed as second-line treatments. This appeared to be the case, as $78 \%$ of our participants reported receiving some form of treatment and, of this $78 \%, 88 \%$ reported that they had self-treated and $29 \%$ reported receiving treatment from a GP or a podiatrist. We considered it unlikely that patients would be willing to be randomised to a treatment that they had previously tried and found to be ineffective, so in order to maximise recruitment to the study we decided to use a $50 \%$ salicylic acid preparation for that arm of the trial.

The $50 \%$ salicylic acid preparation chosen was an OTC medication, available as a 'pharmacyonly' medication, and was used within its marketing authorisation. Although we wished to replicate how this OTC treatment would be delivered in normal practice as far as possible, it was felt that in order to enhance patient safety, participants allocated to salicylic acid should be seen for a safety check at 2 weeks post randomisation. Using a 50\% salicylic acid preparation had the additional benefit that this was a similar concentration to that often used by podiatrists to treat 
verrucae. If the results of the study demonstrated daily patient self-treatment to be more effective than cryotherapy then it would seem likely that podiatrists using a similar concentration of salicylic acid would be able to achieve similar cure rates.

A strength of this study is that it was a pragmatic trial. As far as possible we allowed clinicians to follow their normal practice in terms in delivering of the cryotherapy treatment. Consequently, we asked the clinicians to treat the verrucae as they would in normal practice, with the recommendation that the first freeze should be relatively gentle, in order to assess how well the patient could tolerate the treatment. Subsequent treatments could be more aggressive if it were appropriate and the patient was able to tolerate the treatment. Pooled data from Gibbs $e$ t al.'s systematic review ${ }^{8}$ demonstrated that higher cure rates could be achieved if a more aggressive cryotherapy regimen was used. However, these trials were in different populations and on different types of warts. They also used different definitions of 'aggressive treatment' ranging from one 10 -second freeze to 2 minutes with a cryoprobe. We did originally propose that verrucae should be treated by applying three 10-second applications of liquid nitrogen; however, most health-care professionals reported that this did not reflect their normal practice and that they were unwilling to follow what they considered to be such an aggressive regimen.

In terms of frequency of freezing, evidence from Gibbs et al.'s systematic review ${ }^{8}$ showed no significant difference in long-term cure rates between applying cryotherapy treatments at 2-, 3- or 4-weekly intervals and no significant benefit to prolonging 3-weekly cryotherapy treatments beyond 3 months (approximately four freezes). It was decided that cryotherapy patients should therefore receive a maximum of four treatments, 2-3 weeks apart. Treating at 2- to 3-week intervals allowed the treatment to be delivered followed by a minimum of 3 weeks before the outcome assessment at 12 weeks to allow the participant to heal. This would minimise the possibility of unblinding the outcome assessor to the treatment group.

From the limited data available on adherence to treatment, the salicylic acid patients were applying their salicylic acid for the first 3 weeks. However, the overall amount applied (mean amount applied $2.8 \mathrm{~g}$ ) could suggest that either patients stopped self-treating after the third week or the amount applied during the course of the 8 weeks was relatively small. For some deepseated verrucae, this might not have not been sufficient to clear the verruca.

\section{Patient satisfaction with treatment}

The majority of participants in both groups were happy with their treatment at week 1 and week 3. However, there was a difference in patient satisfaction between the two groups at week 12 . The majority of the cryotherapy group reported that they were happy with their treatment, and only $13 \%$ reported being unhappy. However, the salicylic acid group individuals were less happy with their treatment at week 12 than at previous time points. In addition to this, a larger proportion of salicylic acid patients (31\%) were unwilling to have the same treatment again compared with only $10 \%$ of cryotherapy patients.

\section{Strengths and limitations of the study}

This is a large pragmatic study that recruited patients with longstanding verrucae, the majority of which had been previously treated either by the patient themselves or by a healthcare professional. This is typical of the characteristics of patients presenting to health-care professionals for treatment. 
We were able to undertake a blinded outcome assessment for the primary outcome by a clinician present with the patient at the 12-week visit for the majority of participants and we had to rely on blinded assessment of photographs. However, our experience of using cameras to obtain outcome assessments was not without challenges. First, some sites found it difficult to find the additional time required to take digital photographs during busy clinics and, second, the quality of several photographs was such that an assessment of clearance could not be made. It was anticipated that as the cameras given to sites were a similar make and model to that used successfully on another NIHR HTA-funded trial, ${ }^{24}$ and because members of the EVerT research team owned their own digital cameras and used them to take photographs outside work, the quality of the photographs would not be too great an issue. However, members of the research team encountered difficulties, as they rarely took photographs of such a relatively small scale out of work, and overall a total of 31 out of $190(16 \%)$ photographs were uninterpretable. In an effort to improve the quality of the photographs taken, researchers took several photographs at the same time point and reviewed them on an ongoing basis, taking additional photographs if necessary. However, on several occasions it was noted that although the photographs appeared to be of an acceptable quality on the camera's LCD screen, once uploaded/sent to the YTU the quality of the photograph meant that an assessment was not possible. Further issues were raised in the amount of time it took to send photographs to the YTU. At the time of undertaking the trial it was not possible to upload the photographs directly to the YTU so the photographs had to be sent via e-mail or copied on to a disk and put in the post, both of which were time-consuming. The delay in sending photographs to the YTU, which in some cases was several weeks, meant that it was not possible to always monitor the activity at the site, for example adherence to treatment regimen, as closely as we had first envisaged. Despite these problems, we were still able to achieve blinded outcome data from digital photographs for a total of 159 (69.4\%) participants and overall for 206 (85.8\%) of the 240 trial participants when we combined the blinded clinician assessment at 12 weeks with the blinded photographic assessment.

One limitation of our study is the lack of a no-treatment group, so we were unable to determine the spontaneous clearance rate of verrucae in this population. We did consider having a no-treatment arm to the study, but decided against this for several reasons. First, Gibbs and Harvey's systematic review ${ }^{8}$ showed that salicylic acid was more effective than no treatment, while failing to find any evidence for the effectiveness of cryotherapy. Therefore, the important clinical question was whether or not the use of cryotherapy was superior to that of the salicylic acid treatment. Second, overall recruitment to the study could have been jeopardised, as patients might have been unwilling to be randomised to a no-treatment arm. Finally, a no-treatment arm might have led to bias owing to resentful demoralisation, particularly in those patients in whom the verrucae were painful, longstanding and resistant to previous treatment.

\section{Generalisability of the results}

The EVerT study was a pragmatic trial that recruited from 14 centres across England, Scotland and Ireland. The inclusion of patients recruited from podiatry clinics, from GP practices and from the community means that we can be confident that these results are broadly generalisable and that the study has external validity across the UK and Ireland. However, although the 50\% salicylic acid preparation used in this study was an OTC treatment, it is not the most commonly used concentration and may be viewed as a second-line treatment. Typically, weaker preparations of $15-26 \%$ salicylic acid are used as the first form of treatment and so the results of this study may not mimic the usual clinical situation. Therefore, it is possible that cryotherapy using liquid nitrogen is superior to using these lower concentrations of salicylic acid. However, some GPs are no longer offering cryotherapy using liquid nitrogen as a form of treatment. This is because of the additional treatment cost incurred in order to comply with changes to the health and safety rules 
regarding the storage of liquid nitrogen. Therefore, the availability of the treatment may be lower than that reported in 2002 and, in some cases, may be considered a third-line treatment.

\section{Implications for health care}

There is no evidence from this trial to suggest that cryotherapy used for the treatment of verrucae is more effective than patient self-treatment with $50 \%$ salicylic acid and our economic evaluation concludes that self-treatment with salicylic acid is the most cost-effective option. Our results are applicable only to verrucae or plantar warts. Warts at other sites, such as the hands, may respond differently to cryotherapy.

We evaluated only patient self-treatment with salicylic acid and, therefore, the results cannot be extrapolated to the effectiveness of salicylic acid if it had been delivered by a health-care professional. The freezing agent used for the cryotherapy was liquid nitrogen, so the results from this study cannot be extrapolated to include other freezing agents, such as nitrous oxide, frozen carbon dioxide (dry ice) or OTC freezing treatments, which freeze lesions at higher temperatures.

\section{Implications for research}

Health-care professionals will need to write patient information sheets in such a way to give patients realistic expectations in relation to the effectiveness of cryotherapy treatment.

There are other treatments available for cutaneous warts, but very little good-quality evidence assessing their effectiveness. Further research assessing the effectiveness of these treatments is required in order to inform future practice. 



\section{Acknowledgements}

We would like to thank the participants for taking part in the trial, the podiatrists, GPs and practice nurses for recruiting participants to the study and completing the trial documentation, the principal investigators at each site for co-ordinating participant recruitment, and the TSC and DMEC members for overseeing the study.

We would specifically like to thank:

Peter Arthur, Lucy Bellas, Beverly Brown, Lynne Bryan, Amanda Clark, Andrew Clarke, Michael Concannon, Beryl Cooling, Dawn Curruthers, Chris Davies, Gary Denby, Sean Dinneen, Diane Exley, Lisa Farndon, Simon Gazeley, Elizabeth Green, Stephanie Haughy, Julia Haswell, Christine Hearmon, Christine Howell, Jamil Karolia, Susan Kitchener, Phillip LeDune, Susan Lightfoot, Maria Madigan, Julie Mandehzadeh, Rina Miah, Caroline McIntosh, Christine Northern, Frances Price, Julie Poland, Ilan Rajap, Jayne Robinson, Julie Robinson, Raymond Skinner, Deborah Turner, Susan Walton, Linda White and Catriona Williams, who were members of the research team at each site and recruited participants to the study, as well as Fiona Aitken and Helen Hill from the Medicines for Children Local Research Network, and Kate Biscomb and Kate Wyer from the Primary Care Research Network.

Jill Hall (JH), Farina Hashmi and Jude Watson, who undertook the blinded outcome assessment of clinical verrucae photographs.

\section{Collaborations and contributions of the authors}

The EVerT collaborators (current and past) are:

Sally Baker, Diane Bilton, Stephen Brealey, Ling-Hsiang Chuang, Sarah Cockayne (SC), Sue Collins, Ben Cross, Mike Curran (MC), Sarah Gardner, Farina Hashmi (FH), Catherine Hewitt (CH) Kathryn Hicks (KH), Shalmini Jayakody (SJ), Arthur Kangombe, Nichola McLarnon (NM), Veronica Morton, Jo Orchard, Eugena Stamuli (ES), Kim Thomas (KT), David Torgerson (DT), Gwen Turner (GT), Val Wadsworth, Ian Watt and Gill Worthy (GW).

William Ransom \& Son Plc supplied the Verrugon, plasters and felt pads at no cost, and BOC provided one site with liquid nitrogen storage equipment at a reduced cost.

\section{Statement of independence of researchers}

Neither the Verrugon manufacturers nor BOC had any role in the design of EVerT, or in the collection, analysis and interpretation of data.

DT and JH wrote the original protocol. SC, MC, FH, NM, DT and KT were co-applicants on the HTA application and refined the protocol. IW was the chief investigator and oversaw the study. $\mathrm{SC}$ and $\mathrm{KH}$ were the trial co-ordinators and GT the trial support officer. GW, SJ and $\mathrm{CH}$ designed the clinical analysis. $\mathrm{CH}$ oversaw the conduct of the analysis. SJ conducted the clinical analysis and $\mathrm{AK}$ undertook additional analysis requested by the reviewers. ES designed and undertook the economic analysis. The writing team consisted of SC, $\mathrm{KH}, \mathrm{CH}$, ES and KT, who drafted the report. GD, CM, FH, SJ, DT and IW commented on the report. 


\section{Trial Steering Committee members}

Dr Sam Gibbs, (Independent Chairperson) Consultant Dermatologist, The Great Western Hospital, Swindon.

Dr Jill Mollison (member of the TSC October 2006 to December 2008), Senior Medical Statistician, Centre for Statistics in Medicine, Wolfson College Annexe, University of Oxford, Oxford.

Dr Elaine Thomas (member of the TSC from December 2008 to end), Senior Lecturer in Biostatistics, Arthritis Research Campaign National Primary Care Centre, Keele University, Keele.

Professor Wesley Vernon, Head of Podiatry Service and Research Lead, Sheffield Primary Care Trust, Sheffield.

\section{Data Monitoring and Ethics Committee members}

Dr Anne-Maree Keenan (Independent Chairperson), Assistant Director, NIHR Leeds Musculoskeletal Biomedical Research Unit, University of Leeds, Leeds.

Matthew Hankins, Senior Research Fellow, Division of Public Health \& Primary Care, Brighton \& Sussex Medical School, University of Brighton, Brighton.

Katharine Speaks, Clinical Lead Podiatrist - Diabetes, Centre for Diabetes and Endocrinology, York Hospital, York.

\section{Publications}

Cockayne E. The EVerT (Effective Verruca Treatments) trial protocol: a pragmatic randomised controlled trial to evaluate cryotherapy versus salicyclic acid for the treatment of verrucae. Trials 2010;11:1-5.

Cockayne S. Verrucae trial: call for participants. Podiatry Now 9:July 2006.

Cockayne $\mathrm{S}$, et al., on behalf of the EVerT team. Cryotherapy versus salicylic acid for the treatment of plantar warts (verrucae): a randomised controlled trial. BMJ 2011;342:d3271.

Turner G. Cryotherapy versus salicylic acid for the treatment of verrucae. Society of Chiropodists and Podiatrists. Newsletter, January 2009. 


\section{References}

1. Johnson ML, Roberts J. Skin conditions and related need for medical care among persons 1-74 years. Vital Health Stat 11 1978;1660:1-26.

2. Rea JN, Newhouse ML, Halil T. Skin disease in Lambeth. A community study of prevalence and use of medical care. Br J Prev Soc Med 1976;30:107-14.

3. Beliaeva TL. The population incidence of warts. Vestn Dermatol Vener 1990;2:55-8.

4. Williams HC, Pottier A, Strachan D. The descriptive epidemiology of warts in British schoolchildren. Br J Dermatol 1993;128:504-11.

5. Kilkenny M, Merlin K, Young R, Marks R. The prevalence of common skin conditions in Australian school students. 1. Common plane and plantar viral warts. $\mathrm{Br} J$ Dermatol 1998;138:840-5.

6. van Haalen FM, Bruggink SC, Gussekloo J, Assendelft WJ, Eekhof JA. Warts in primary schoolchildren: prevalence and relation with environmental factors. $\mathrm{Br} J$ Dermatol 2009;161:148-52.

7. Massing AM. Natural history of warts. Arch Dermatol 1963;87:306-10.

8. Gibbs S, Harvey I. Topical treatments for cutaneous warts. Cochrane Database Syst Rev 2006;3:CD001781.

9. Sterling JC, Handfield-Jones S, Hudson PM. Guidelines for the management of cutaneous warts. Br J Dermatol 2001;144:4-11.

10. Clinical Knowledge Summaries. Warts and verrucae. January 2009. URL: www.cks.nhs.uk/ warts_and_verrucae\# (accessed May 2010).

11. Johnson LW. Communal showers and the risk of plantar warts. J Fam Pract 1995;40:136-8.

12. Health Protection Agency (HPA). School health matters: a guide to communicable diseases and infection control. London: HPA; 2008. URL: www.hpa.org.uk (accessed May 2010).

13. Schofield J, Grindlay D, Williams HC. Skin conditions in the UK: a health care needs assessment. Nottingham: Centre of Evidence Based Dermatology; 2009.

14. Thomas KS, Keogh-Brown MR, Chalmers JR, Fordham RJ, Holland RC, Armstrong SJ, et al. Effectiveness and cost-effectiveness of salicylic acid and cryotherapy for cutaneous warts. An economic decision model. Health Technol Assess 2006;10(25).

15. Bruggink SC, Gussekloo J, Zaaijer K, Assendelft WJJ, Berger MY, Koes BW, et al. Warts: cryotherapy, salicylic acid or expectantly awaiting? a randomised controlled trial. J Invest Dermatol 2008;128:8.

16. Drummond MF, O'Brien B, Stoddart GL, Torrance. Methods for the economic evaluation of health care programmes. 2nd edn. Oxford: Oxford University Press; 1997.

17. BOC. URL: www.boconline.co.uk/ (accessed June 2010).

18. Personal Social Service Research Unit (PSSRU). Unit costs of health and social care 2009. Canterbury: University of Kent; 2009.

19. Nu-care Products. URL: www.nu-careproducts.co.uk/chiropody.htm\#feltpads, product PPD126 (accessed June 2010).

20. Boots. URL: www.boots.com/en/Boots-Fabric-Strip-10-pack_5466/ (accessed June 2010). 
21. BOC Products. URL: www.bocsafetyproducts.co.uk/main/home/index.aspx (accessed June 2010).

22. British Medical Association and Royal Pharmaceutical Society of Great Britain. British national formulary. No. 59, March 2010. London: BMA and RPS; 2010.

23. Edwards P, Roberts I, Clarke M, DiGuiseppi C, Pratap S, Wentz R, et al. Methods to increase response rates to postal questionnaires. Cochrane Database Syst Rev (Online). 2007;2:MR000008.

24. Dumville J, Worthy G, Soares M, Bland JM, Cullum N, Dowson C, et al. VenUS II: a randomised controlled trial of larval therapy in the management of leg ulcers. Health Technol Assess 2009:13(55).

25. Bunney M, Nolan M, Williams D. An assessment of methods of treating viral warts by comparative treatment trials based on a standard design. Br J Dermatol 1976;94:667-79.

26. Steele K, Irwin W. Liquid nitrogen and salicylic/lactic acid paint in the treatment of cutaneous warts in general practice. J Roy Coll Gen Pract 1988;38:256. 


\section{Appendix 1}

\section{Regulatory approvals}

\begin{tabular}{|c|c|c|c|c|}
\hline Research site & Ethical site assessor & $\begin{array}{l}\text { Date of favourable } \\
\text { ethical opinion }\end{array}$ & R\&D approval & $\begin{array}{r}\text { Competent } \\
\text { authority approval }\end{array}$ \\
\hline $\begin{array}{l}\text { School of Podiatry, Centre for } \\
\text { Healthcare Education School of } \\
\text { Podiatry, University of Northampton }{ }^{\mathrm{a}}\end{array}$ & $\begin{array}{l}\text { Leicestershire, } \\
\text { Northamptonshire \& Rutland } \\
\text { REC } 2\end{array}$ & 26 October 2004 & $\mathrm{~N} / \mathrm{A}$ & 8 February 2005 \\
\hline $\begin{array}{l}\text { With approval to contact GPs with } \\
\text { the PCT Leicestershire Primary Care } \\
\text { Alliance }\end{array}$ & & 6 November 2006 & 4 January 2007 & \\
\hline $\begin{array}{l}\text { University of Brighton (Leaf Hospital) } \\
\text { and Eastbourne PCT Sussex NHS } \\
\text { Research Consortium }\end{array}$ & East Sussex LREC & 23 September 2006 & 24 October 2006 & 10 August 2006 \\
\hline $\begin{array}{l}\text { Southern General Hospital, South } \\
\text { University Division, Greater Glasgow } \\
\text { and Clyde Health Board and } \\
\text { Glasgow Caledonian University }\end{array}$ & South Glasgow and Clyde REC & 29 March 2007 & 21 December 2007 & 13 November 2007 \\
\hline $\begin{array}{l}\text { Huddersfield University, Division of } \\
\text { Podiatry, Department of Clinical and } \\
\text { Health Sciences, Huddersfield }{ }^{a}\end{array}$ & Airedale REC & $\begin{array}{r}5 \text { May } 2007^{b} \\
3 \text { December } 2008^{b}\end{array}$ & 15 June 2007 & 13 November 2006 \\
\hline Brownlow Group Practice, Liverpool & Liverpool Paediatric REC & 13 May 2007 & 19 September 2007 & 26 June 2007 \\
\hline Springfield Surgery, Bingley & Bradford REC & 5 December 2007 & 21 November 2007 & 12 October 2007 \\
\hline Sheffield PCT & North Sheffield LREC & 3 April 2008 & 28 August 2008 & 23 April 2008 \\
\hline $\begin{array}{l}\text { Claughton Medical Centre, } \\
\text { Birkenhead }\end{array}$ & Cheshire REC & 3 November 2008 & 10 March 2009 & 9 July 2008 \\
\hline $\begin{array}{l}\text { Arlington Road GP Surgery, } \\
\text { Eastbourne }\end{array}$ & Brighton East REC & 23 December 2008 & 23 January 2009 & 4 December 2008 \\
\hline Harbinson House, Sedgefield & $\begin{array}{l}\text { Research Management \& } \\
\text { Governance Unit of County } \\
\text { Durham \& Tees Valley PCTs }\end{array}$ & 6 October 2009 & 6 October 2009 & 16 October 2009 \\
\hline Sacriston Surgery, Sacriston & $\begin{array}{l}\text { Research Management \& } \\
\text { Governance Unit of County } \\
\text { Durham \& Tees Valley PCTs }\end{array}$ & 7 August 2009 & 28 July 2009 & 29 July 2009 \\
\hline $\begin{array}{l}\text { Peaseway Medical Centre, Newton } \\
\text { Aycliffe }\end{array}$ & $\begin{array}{l}\text { Research Management \& } \\
\text { Governance Unit of County } \\
\text { Durham \& Tees Valley PCTs }\end{array}$ & 7 August 2009 & 28 July 2009 & 29 July 2009 \\
\hline The Haven Surgery, Burnhope & $\begin{array}{l}\text { Research Management \& } \\
\text { Governance Unit of County } \\
\text { Durham \& Tees Valley PCTs }\end{array}$ & 7 August 2009 & 28 July 2009 & 29 July 2009 \\
\hline $\begin{array}{l}\text { The National University of Ireland, }{ }^{\text {a }} \\
\text { Galway }\end{array}$ & Galway REC & 20 March 2009 & $\mathrm{~N} / \mathrm{A}$ & 30 January 2009 \\
\hline
\end{tabular}

N/A, not applicable; R\&D, research and development; REC, research ethics committee.

a Non-NHS site.

b Approval for change in principal investigator.

Approval was gained at two additional sites; neither was able to start recruitment. 



\section{Appendix 2}

\section{Details of the study sites}

$\mathrm{T}_{\mathrm{h}}$

he following sites recruited at least one patient:

- The University of Northampton Podiatry School Clinic, Northampton

- The University of Huddersfield Podiatry School Clinic, Huddersfield

- The University of Brighton Podiatry School Clinic at the Leaf Hospital, Eastbourne

- Southern General Hospital, Glasgow/Glasgow Caledonian University Podiatry School, Glasgow

- The National University of Ireland, Galway (NUI Galway) Podiatry School Clinic, Galway

- Brownlow Group Practice, Liverpool

- Springfield Surgery, Bingley

- Sheffield Primary Care Trust Podiatry Clinic, Sheffield

- Sacriston Surgery, Sacriston

- The Haven Surgery, Burnhope

- Peaseway Medical Centre, Newton Aycliffe

- Arlington Road Medical Practice, Eastbourne

- Claughton Medical Centre, Birkenhead

- Harbinson House Surgery, Sedgefield.

Approval was gained and an initiation visit was performed at the following two sites, but neither recruited any patients:

- Annfield Plain Surgery, Stanley, Co. Durham

- Islington Primary Care Trust Services, London. 



\section{Appendix 3}

\section{Patient information sheets and consent form}




\title{
Patient information sheets
}

\section{THE UNIVERsiTy of Work}

Local headed paper

\section{DEPARTMENT OF HEALTH SCIENCES}

\section{A STUDY OF DIFFERENT TYPES OF TREATMENT FOR VERRUCAE}

\author{
Information Sheet \\ Version 716 Oct 2008
}

You are being invited to take part in a research study, which aims to find out the best way to treat verrucae. Before you decide if you would like to take part you will need to understand why the research is being done and what it will involve. We would be grateful if you would read the following information and discuss it with your family and friends if you wish. Please ask if there is anything that is unclear or if you need more information and take time to decide whether or not you would like to take part.

\section{What is the purpose of this study?}

Verrucae are a common, infectious and sometimes painful problem. Most verrucae will disappear spontaneously after 6 to 12 months without treatment. However, patients may seek treatment from a podiatrist/GP/other Health Care professional if their verruca is painful or because they are being prevented from doing sports. There are many different ways to treat verrucae but it is unclear which treatment is best. The purpose of this study is to compare two of those treatments, an acid paste which you can buy over the counter from a pharmacist and a freezing technique, which is currently used to treat verrucae within the Podiatry Department/GP practice/other clinic at (insert name of specific site). We want to find out which is the best treatment to cure verrucae and what you thought about the treatment. We are also interested to know how much the treatments costs.

\section{Who is carrying out the research?}

This is a joint research project between the Podiatry Department/GP clinic/other clinic at (insert name of specific site) and the York Trials Unit. Qualified HCP at the clinic led by (HCP name) will treat all the patients. Two researchers, (name of researchers) from the Trials Unit at York University will collect and analyse the data.

\section{Who is funding the research?}

The NHS Health Technology Assessment Programme is paying for the research. 


\section{Why have I been chosen?}

We are inviting all patients attending the Podiatry Department at GP practice/other clinic (insert name of specific site) who have a verruca to participate in this study. We hope to study 266 patients in total.

\section{Do I have to take part?}

It is completely up to you if you would like to take part. If you do decide to take part you will need to sign the consent form. For patients under the age of 16 a parent or guardian will be asked to sign as well. If you decide to take part you are still free to withdraw at any time and without giving a reason. A decision to withdraw at any time or a decision not to take part, will not affect the standard of care you receive.

\section{What will happen to me if I take part?}

If you wish to take part you will need to complete the questionnaire and consent form and take it with you when you attend the podiatrist/GP/ practice nurse/other health care professional for your first appointment. Because we do not know which of the two treatments is best we need to make comparisons by putting patients into two different groups. Which group you are put in depends on chance and is rather like tossing a coin. You will have a 50:50 chance of getting either treatment. Patients will have been sent an appointment by the podiatry clinic to have their verruca treated along with this information. All patients will be seen by the podiatrist/ GP/practice nurse/other health care professional at their first appointment. Those assigned to the salicylic acid paste treatment will be shown how to apply it. You will then be asked to take the treatment home with you and apply it daily up to a maximum of 8 weeks. We will also ask you to attend a further appointment in two week's time. Those assigned to the cryotherapy group will be required to attend follow-up appointments as required when the verruca will be re-treated if necessary depending on your verruca. At 12 weeks after the first treatment, all patients will be asked to attend for a final assessment of whether their verruca has been cured, even if their verruca has been cured before this time. We will take a photograph of your verruca at the start of the study and then regularly to see if your verruca is reducing in size. In this study it is important that the podiatrist carrying out this assessment remains unaware of the treatment you have received. We will therefore ask you not to mention or talk about the treatments you have received during the trial to the person carrying out this assessment. In order to help cover your travel costs to take part in this trial, we will reimburse you $£ 5 / £ 10$ for each visit up to the 12 week visit you make for treatment for your verruca and $£ 20$ for the 12 week visit. You will also be sent four further questionnaires by the University of York at 1, 3, 12 and 24 weeks after you agreed to take part in the study. You can choose to complete either paper or on-line versions of these questionnaires. If after 12 weeks your verruca has not cleared up at this stage, the podiatrist will advise you of the best course of action, which may include further treatment.

\section{What do the two types of treatment involve?}

The first treatment involves the application of an over the counter preparation of a salicylic acid paste to the verruca. The podiatrist/GP/nurse/other healthcare professional will show you how to apply the paste at your first appointment. You will then be given the treatment to take home with you and asked to apply it daily up to a maximum of 8 weeks. The second option is the application of liquid nitrogen to the verruca tissue for ten to twenty seconds each treatment, and again this will be repeated every two weeks for a maximum of four treatments. The area will be padded after treatment and you will be advised how to care for the foot after treatment. 
What are the side effects of any treatment received when taking part?

Occasionally, people report mild discomfort either during or after treatment. If this happens then report it to the podiatrist who will advise you how best to deal with this. If you become in any way concerned then contact (name of podiatrist/ GP practice nurse/other health care professional, Podiatrist/GP/practice nurse/other type of health care professional on tel (insert telephone number).

\section{What are the possible benefits of taking part?}

We hope that both the treatments will help you. However, this cannot be guaranteed. The information we get from this study may help us to treat future patients with verrucae better.

\section{What happens when the research study stops?}

You will still receive treatment after the study has stopped, if this is necessary. The podiatrist will consult with you on the best course of action.

\section{What if something goes wrong?}

If you are harmed by taking part in this research project, there are no special compensation arrangements. If you are harmed due to someone's negligence, then you may have grounds for a legal action but you may have to pay for it. Regardless of this, if you wish to complain, or have any concerns about any aspect of the way you have been approached or treated during the course of this study, the University of York Trial's Unit complaints mechanisms will be available to you, alternatively the normal National Health Service complaints mechanisms may be available to you.

\section{Will my taking part in this study be kept confidential?}

All information which is collected about you during the course of the research will be kept strictly confidential, both at the clinic where you receive treatment and at the University of York. This will be in accordance with the Data Protection Act 1998. Your General Practitioner will be notified that you are taking part in the study. Study information must be made available to the Medicines and Healthcare Products Regulatory Agency, which supervises drug trials in the UK, and the relevant ethics committees in the UK. Representatives of these bodies may also examine your hospital or clinical records and, by signing the consent form, you are giving permission for these records to be examined. These organisations have strict policies regarding confidentiality. No records bearing your name will leave the hospital/clinic where you take part in the study. Your study data, that will be transmitted to the York Trials Unit for analysis, will be identified by a Patient ID Number only.

\section{What will happen to the results of the research study?}

All the participants in the study will be personally informed about the results once the study is completed. It is intended to publish the results in approximately Autumn 2010 in a suitable medical journal. If participants wish to obtain a copy of the published results they should contact the podiatry clinic for details. Individual participants will not be identified in any publication.

\section{What do I do if I don't want to take part in this study?}

No problem, when you attend the clinic to see the podiatrist your treatment will not be affected by this. However, even if you do not want to take part in our study we would very much like you to fill in the questionnaire and return it to the podiatrist when you attend for 
treatment because we would like to know about the health of all people with verrucae. (You do not have to give personal details if you would prefer not to).

Who has reviewed the study?

This study has been reviewed and approved by Trent Multi Research Ethics Committee. All research that involves NHS patients or staff, information from NHS medical records or uses NHS premises or facilities must be approved by a NHS Research Ethics Committee before it goes ahead. Approval does not guarantee that you will not come to any harm if you take part. However, approval means that the Committee is satisfied that your rights will be respected, that any risks have been reduced to a minimum and balanced against possible benefits, and that you have been given sufficient information on which to make an informed decision to take part or not.

What do I do if I do want to take part in this study?

If you are interested in taking part please complete the enclosed questionnaire and sign the consent form, returning it to the podiatrist when you attend for your first appointment.

\title{
Where can I get further information about the study?
}

If you require any further help or information please do not hesitate to contact either (Podiatrist/GP/practice nurse/other health care professional name) the podiatrist/GP/practice nurse/other health care professional telephone number) or researcher's contact details.

\section{What if I have any concerns?}

If you have any concerns or other questions about this study or the way it has been carried out, you should contact the investigator [name etc], or you may contact the hospital/PCT [name etc] complaints department or the trial coordinator (name of trial coordinator).

\section{THANK YOU FOR TAKING THE TIME TO READ ABOUT THIS STUDY}

\author{
https://www.hsytu.york.ac.uk/verruca/login.aspx
}

https://www.verrucatrial.co.uk 


\title{
Patient information sheet for children
}

\author{
THE UNIVERSITY of Work \\ (Information leaflet version 4 25/07/2007 for children over 12) \\ Local headed paper \\ Invitation
}

\section{The project on "treating verrucae"}

Hello

My name is (name of researcher) and I work at the University of York looking at different ways of removing verrucae. This leaflet is to invite you to take part in a project which is looking at two common ways of treating verrucae. At the moment I am are not sure which is the best way, but doing this project should help me find out.

\section{Why am I writing to you?}

I am inviting everyone with a verruca who is going to the clinic to see if they would like to take part in this project. We hope 266 people will take part.

\section{How did you get my name and address?}

If you get a leaflet from me it is because the person caring for you has given me permission to contact you.

\section{What is the point of the project?}

The information from the project can help in three ways:

It will tell me which of these two ways is best at curing a verruca.

It will give me an idea if one treatment costs more than the other.

It will tell me what you thought about the treatment you had.

\section{What will I have to do?}

If you want to take part, you simply fill in the questionnaire and consent form and give it to the person who is treating your verruca. They will arrange for you to come to the clinic and at the end of 12 weeks will look to see if your verruca has gone. We will take a photograph of your verruca at the start of the study and then regularly to see if your verruca is reducing in size.

I will send you four more questionnaires to fill in to find out how you felt about the treatment you had, how many times you had to go to the clinic and about how you got there. If you like you can choose to fill these questionnaires in on-line. 


\section{What are the treatments being used in this study?}

There are lots of different ways of treating verruca, but in this study we are using two different treatments, a freezing method and an ointment.

If your verruca is frozen, then your podiatrist/GP/nurse/ other HCP will apply a very cold liquid called nitrogen, to your verruca until it is frozen. Depending on its size this may take up to 30 seconds to freeze. You may be asked to come back to the clinic for a maximum of four treatments. But there will be a two/three week gap in between each treatment.

The other treatment we are testing is an ointment. There are several different ointments available but in this study we are using one called Verrugon. Your podiatrist/GP/nurse/ other HCP will tell you and the person caring for you how to apply it safely and give you some to take home with you. You may be asked to apply it every day for a maximum of eight weeks. We will ask you to come back to the clinic two weeks after you started in the study to make sure you are not having any problems and to give you some more ointment if you need it.

We will ask everyone to come back to the clinic 12 weeks after they began the study to see if their verruca has gone and to take a photograph of it.

\section{What treatment will I get?}

Which treatment you get is a matter of chance. It is like tossing a coin to decide which of the treatments you will get. This makes it a fair test.

\section{What happens to the information?}

All the information you send back to me is put into a computer where we will be able to see which is the best way to remove verrucae.

\section{Will anyone else be told what I say?}

No, everything in the questionnaire is confidential. Your name will not be used in any articles we write about this project.

\section{Do I have to take part in the project?}

No, you do not have to take part in the project. If you decide not to take part, I will accept your decision and I will not ask you to give a reason. 


\section{What happens next?}

If you decide you would like to take part in this project, please fill in the forms with this letter and take it with you and give it to the person who is treating your verruca.

Thank you for reading this letter.

Name of researcher

If you want to contact me to talk about the project, telephone (insert telephone number) between 9:30am and 2:00pm Monday to Friday. If I am not there please leave a message on the answer phone. 


\section{Consent form}

Version 5 16/10/2008

\section{CONSENT FORM}

Title of Study: A Study of Different Types of Treatment for Verrucae.

Investigator's Name: (Podiatrist's Name, position and name of site)

Please initial the boxes.

1. I confirm that I have read and understand the information sheet version [insert number] dated [insert date], or for children under the age of 16 version [insert number] dated [insert date] for the above study and have had the opportunity to ask questions.

2. I understand that my participation is voluntary and that I am free to withdraw at any time, without giving any reason, without my medical care or legal rights being affected
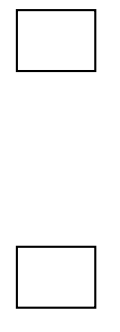

3. I understand that sections of any of my medical notes may be looked at by responsible individuals from the podiatry department/GP practice at (name of centre) or other members of the NHS Trust, representatives of the Study's Sponsor (university of York) and regulatory authorities, where it is relevant to my taking part in research. I give permission for these individuals to have access to my records.

4. I understand that my General Practitioner will be informed that I have taken part in this study.

5. I agree to take part in the above study.

6. I agree to have my verruca photographed.

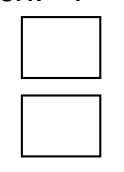




\section{Cryotherapy patient's advice sheet}

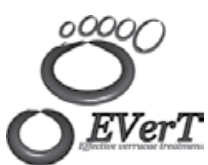

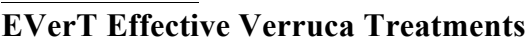

\section{What is cryotherapy?}

Cryotherapy is used to treat various skin conditions, including warts on the body and verrucae on the feet (both caused by versions of the human papilloma virus).

The treatment causes a skin irritation or a surface wound, through the application of liquid nitrogen which briefly freezes the skin. This is either by means of a fine spray to the area, or by applying liquid nitrogen directly using a probe or cotton bud tip. The treatment aims to trigger a response from your immune system, to this and all other warts or verrucae which you may have.

Application of liquid nitrogen onto the skin can be briefly uncomfortable, due to the extreme cold when the area is frozen. This is normal. The length of time of freezing will depend on the depth and size of verruca being treated, the duration being agreed between you and your clinician beforehand.

Usually a 10 second freeze is agreed, though often shorter for initial sessions in order to assess your body's reaction to cold. However, you can halt the treatment at any point, if it becomes too uncomfortable for you.

\section{What can I expect afterwards?}

Depending on the length of freezing time, the skin may show no reaction, or some reddening, or occasionally it may develop a blister or a deep bruise (as with a burn from heat). This is normal. The area may also feel a little uncomfortable after treatment. Depending upon its location, your clinician may choose to pad the area to promote comfort, or to tape it in order to deter the development of a blister (making a bruise more likely).

In all cases, you should keep the area as clean and dry as possible for the following 24 hours, in order to deter any infection at the site. After 24 hours, remove the pad or dressing and inspect the area yourself. If there appears to be an open wound (with a possibility of infection), then continue protecting the area with sticking plasters until it has healed. If the surface of the skin appears intact, you may continue with your normal activities without any further dressings. 
Your clinician will arrange a review appointment with you after cryotherapy to check the area and repeat the treatment as appropriate. It is important that you attend this appointment.

In the unlikely event that you experience excessive pain or a weeping discharge at the treatment site, or you have any concerns about the treated area, please contact the clinic for advice.

In the first instance please contact:

Tel:

If unavailable please contact the podiatry clinic on:

Tel:

where reception staff will be able to put you in contact with another clinician for advice.

Insert web site details 


\section{Acid therapy patient's advice sheet}

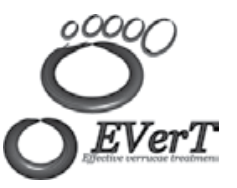

EVerT Effective Verruca Treatments

Insert trust logo

What is acid therapy?

Salicylic acid is used to treat various skin conditions, including warts on the body and verrucae on the feet (both caused by versions of the human papilloma virus).

The treatment causes a skin irritation or a surface wound, through the application of an acid which dehydrates and damages the surface of the skin. The acid can be of various strengths, from 5 - 10\% (Bazuka) to $50 \%$ (Verrugon). The treatment aims to trigger a response from your immune system, to the treated wart and all other warts or verrucae which you may have.

It is rare for application of salicylic acid to the skin to cause any pain or discomfort. However, if an extreme itching, reddening, allergy reaction does occur, the acid can be washed away with water. You should also wash your hands after applying the acid, so as to prevent its accidental rubbing into your eyes (very painful).

Care must be taken to avoid damaging the good skin which surrounds the wart or verruca, since this is where the replacement skin originates from. You only need to apply sufficient acid to cover the surface of the skin, particularly underneath feet where pressure causes any excess acid to spread onto surrounding areas.

\section{How do I apply Verrugon?}

Verrugon is suitable for application at home by adults, and can be used with children under adult supervision. Please read the details on the Verrugon box.

1. Prior to treatment, any rough skin covering the verruca which is proud of the surrounding skin should be removed. Use the small personal emery board supplied with the Verrugon kit, or additional ones from your local pharmacist, to file down the surface of the verruca. However, be careful not to graze surrounding skin with the file, as this could spread the verruca. 
2. As instructed by your clinician, place the hole of the felt pad above the selected, treatable verruca. It is unusual to treat verrucae which are close to joints or the nails. In the case of a large or mosaic wart, centre the hole over an identifiable edge where the active virus is closer to the surface.

3. It is not necessary, nor is it desirable to cover a large area of skin with salicylic acid. You should apply acid to the verrucous skin at the bottom of the hole in the felt pad - only sufficient to cover the skin. The felt is intended to protect the surrounding good skin. However, if you fill the hole with acid, the whole pad becomes an acid reservoir and too much skin damage may be caused.

4. Cover the felt pad and acid treatment with one of the waterproof sticking plasters provided with the Verrugon kit. The dressing enhances the penetrative effect of the acid and helps to keep it in place.

5. Now wash your hands.

The Verrugon kit provides enough felt and plasters to treat your verruca for nine successive days. You should treat the same wart and location, day after day. Be careful not to damage the surrounding skin.

\section{What can I expect afterwards?}

Some reddening of the skin may result from repeated use of the salicylic acid treatment. If it becomes tender, sore or inflamed, wash away the acid with plain water and rest the area from further treatment until the situation has resolved.

Your clinician will arrange a review appointment with you to check the area and your progress. It is important you attend this appointment. Additional felt rings and waterproof plasters may be provided at this time.

In the unlikely event that you experience excessive pain or a weeping discharge at the treatment site, or you have any concerns about the treated area, please contact the clinic for advice. 
In the first instance please contact:

Tel:

If unavailable please contact the podiatry clinic on:

Tel:

where reception staff will be able to put you in contact with another clinician for advice.

(Insert web site address) 


\section{Appendix 4}

\section{Data collection forms}


Baseline patient questionnaire

\section{THe University of York}

\section{A STUDY OF DIFFERENT TYPES OF \\ TREATMENT FOR VERRUCAE}

\section{BASELINE QUESTIONNAIRE}

Participant Number:

(For office use only)

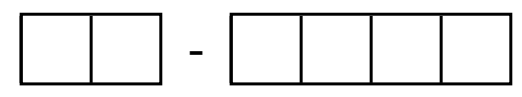




\section{PLEASE READ ALL THE INSTRUCTIONS BEFORE COMPLETING THE QUESTIONNAIRE}

Thank you for agreeing to take part in this evaluation.

Please answer ALL the questions. Although it may seem that questions are asked more than once, it is still important that you answer every one.

If you find it difficult to answer a question, do the best you can.

Please follow the instructions for each section carefully.

For each section, if you are asked to put a cross in the box, please use a cross rather than a tick, as if you were filling out a ballot paper.

For example in the following question, if your answer to the question is yes, you should place a cross firmly in the box next to yes.

Do you drive a car? Yes $\bigotimes$

No

If you are asked to circle a number, please use a circle rather than underlining a number.

For example, in the following question if you are asked 'how happy are you today?' where ' 1 ' is 'very unhappy' and ' 5 ' is 'very happy', if you feel neither happy nor unhappy you may wish to answer 3 . You do this by clearly circling the number 3.

Very

unhappy

PLEASE USE A BLACK OR BLUE PEN.

Please do not use a pencil or any other coloured pen.

Please read all the instructions for each section. 
Please complete all the sections in this questionnaire. Thank you.

Please enter the date you are completing this questionnaire:
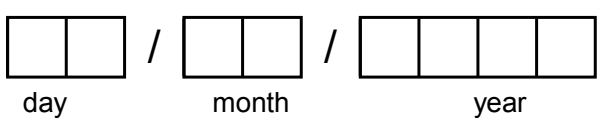

\section{This section asks about your verruca}

1. How long have you had your current verruca?

(Please state in months and weeks)

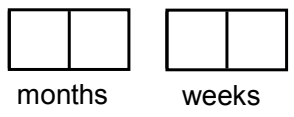

2. Have you had any previous treatment for this verruca? (Please cross one box)

Yes

No

2a. If 'YES' please cross all that apply

Self-treatment using a preparation bought over the counter

Treatment from a podiatrist/ chiropodist

Treatment from your GP

Participated in a trial investigating different treatments of verrucae
If Yes,

please specify

If Yes,

please specify

Other treatment, please specify

If other trial, please specify treatment

Other types of treatment, please specify

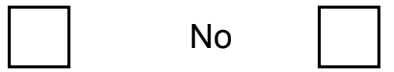

3. What are the reasons for seeking treatment for this verruca? (Please cross all that apply)

The verruca is painful

It stops me from going swimming

It stops me from doing other sports

Other

If other,

please specify

4. How painful is your verruca today? (please circle one number only)

Not at all

0
A little bit

1
Moderately

2
Quite a lot

3
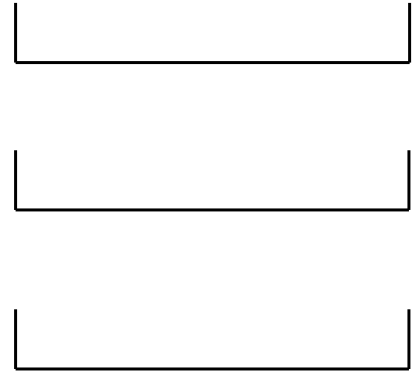

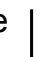


5. Before this verruca, have you had any others?

Yes

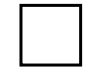

No

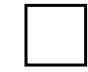

Don't know

5a. If you had a verruca before, how many have you had?

5b. How old were you when you had your last verruca?
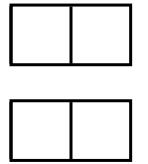

\section{This section asks about your preferences}

1. If you take part in the trial, we would like you to fill in some more questionnaires. How would you like to fill in these questionnaires? (Please cross one box only)

Please send me paper copies like this one, in the post

I would like to fill the questionnaire in on-line

2. If you take part in the trial, we may wish to contact you for example to remind you to fill in a questionnaire or ask you if your verruca has gone. Please tell us how you would like us to contact you? (Please cross all that apply)

By post

By text

If text, please write your mobile telephone number here

By email

If email, please write your email address here

This section asks about your personal details

What is your date of birth?
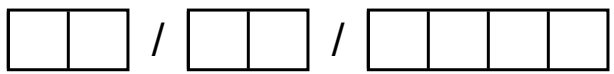

Are you?

Male

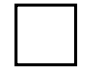

Female

When is your appointment with the podiatrist? (The date of your appointment will have been sent to you with this information pack.)

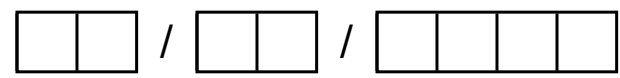

IF YOU WISH TO TAKE PART IN THIS STUDY PLEASE COMPLETE THE ENCLOSED CONSENT FORM IF YOU DO NOT WISH TO TAKE PART IN THIS STUDY WE WOULD STILL APPRECIATE YOU RETURNING THIS COMPLETED QUESTIONNAIRE.

THANK YOU FOR TAKING THE TIME TO COMPLETE THIS QUESTIONNAIRE.

https://www.hsytu.york.ac.uk/verruca/login.aspx 
Date form completed

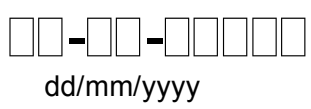

\title{
A STUDY OF DIFFERENT TYPES OF TREATMENT FOR VERRUCAE
}

\author{
Randomisation Form
}

Patient's trial number

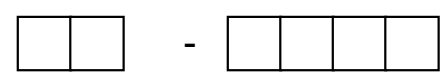

\section{Trial Centre}

(Please cross 1 box only)
Eastbourne Leaf Hospital

Glasgow Caledonian Podiatry School

Northampton Podiatry School

Huddersfield Podiatry School

The Arlington Road Medical Practice Eastbourne

Springfield Surgery Bingley

Claughton Medical Centre

Sheffield PCT

Galway - National University of Ireland

Sacriston Surgery

Peaseway Medical Centre

The Haven Surgery

Annfield Plain Surgery

Harbinson House

\section{Consent criteria}

1. Is the patient able to provide informed consent?
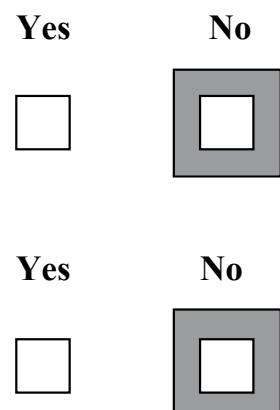

2. Has the patient provided informed written consent to entering the trial? i.e. have they read and understood the patient information sheet and signed the patient consent form? 


\section{Inclusion criteria}

1. Is the patient aged 12 or over?

2. Does the patient have a verruca which can be treated with both salicylic acid and cryotherapy?

\section{Exclusion criteria}

1. Does the patient have impaired healing eg due to diabetes, peripheral vascular disease?

2. Is the patient currently participating in another trial for the treatment of their verrucae?

3. Is the patient immunosuppressed (eg has agammaglobulinaemia) or currently taking immunosuppressant drugs such as oral corticosteroids?

4. Is the patient currently on renal dialysis?

5. Does the patient have cold intolerance? (eg Raynaud's syndrome or cold urticaria)

6. Does the patient have any of the following conditions: Blood dyscrasias of unknown origin, cryoglobulinaemia, cryofibrinogenaemia, collagen and auto-immune disease?

7. Does the patient have neuropathy?
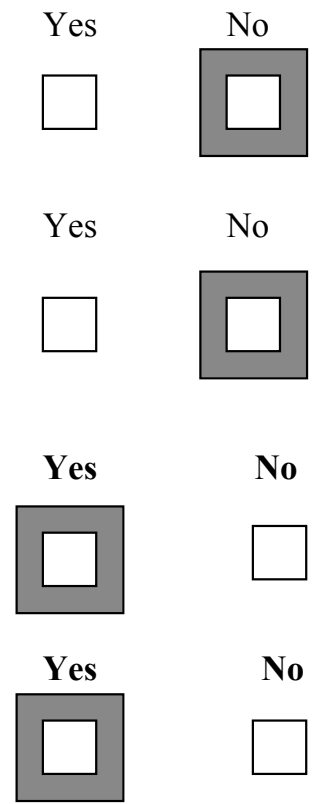

No

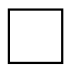

No
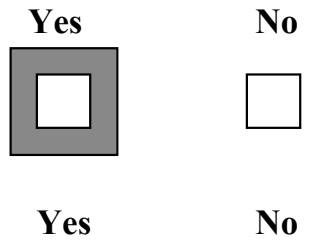

No
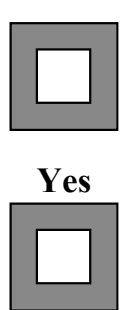

No
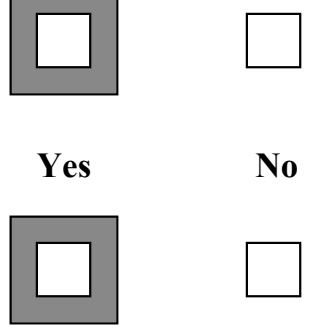

No

Yes No
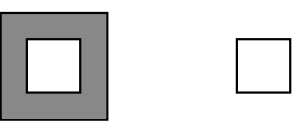

\section{If any of the responses fall into the grey boxes then the patient is} NOT ELIGIBLE for the trial. 


\section{Patient details}

Patient's title:

Patient's full name:

Patient's address:

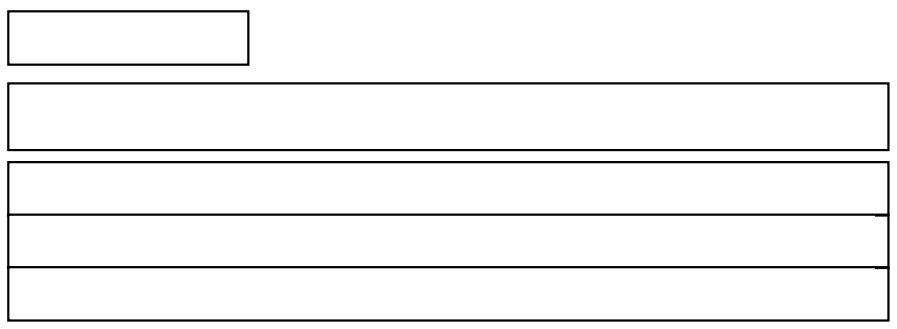

Patient's postcode:

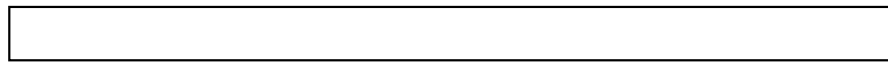

Patient's date of birth

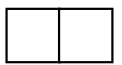

day
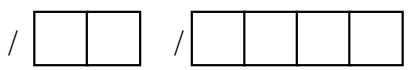

month year

Patient's telephone number:

Name of patient's GP:

GP's address:

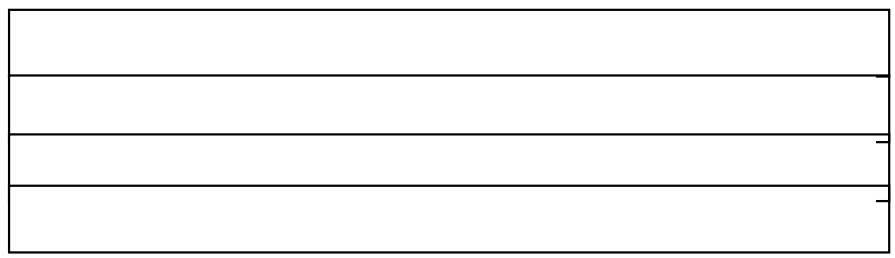

Parent/guardian details for patients aged under 16

Parent/Guardian's title:

Parent/Guardian's full name:
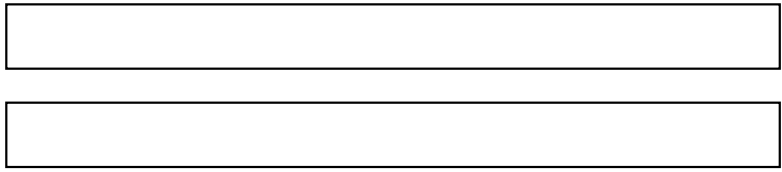

Does the parent/guardian live at the same address as the patient? Yes

No

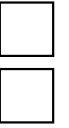

If no, please give details:

Parent/guardian's address:
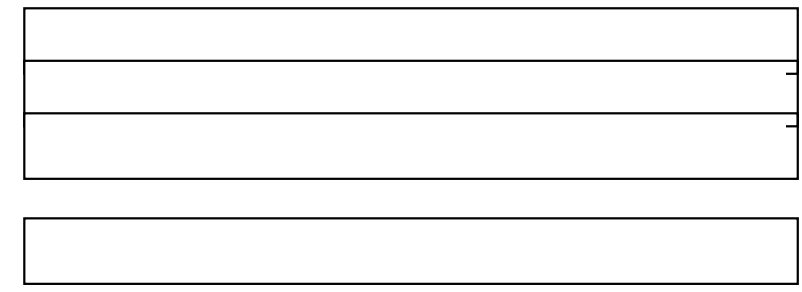
Parent/guardian's postcode:

Parent/guardian's telephone number:

The participant is due to fill in another questionnaire in one week. It would be useful if you could state how they would prefer to complete this?

Postal

On-line

(This information can be found on the patient's baseline questionnaire)

Once all of these questions are complete please call the telephone randomisation service on 08000566682 between 09:00 and 17:00 Monday to Friday, and then complete the allocation details on the following page according to the details given by the telephonist.

\section{Allocation details}

The patient has been assigned to:

$50 \%$ salicylic acid

(Please place a cross in the appropriate box)

Cryotherapy using liquid nitrogen

Your name

Your signature

\section{Digital photograph reminder}

You will be prompted to remember to take a photo of the verruca before you treat the patient.

\section{Please file this form with the patient's notes. Thank you.}




\section{Patient ineligible form}

\section{THE UNIVERSITY of York \\ A STUDY OF DIFFERENT TYPES OF TREATMENT FOR VERRUCAE INELIGIBLE PATIENT FORM}

Patient ID number:

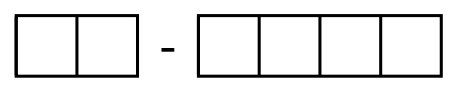

Please complete this form if you see a patient who would like to have taken part in the trial but who was not eligible. (It is not necessary to give the patient's name).

\section{Patient details:}

Date patient considered for the trial:

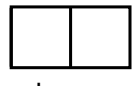

day

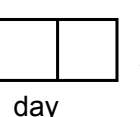

day

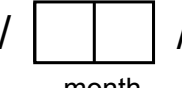

month
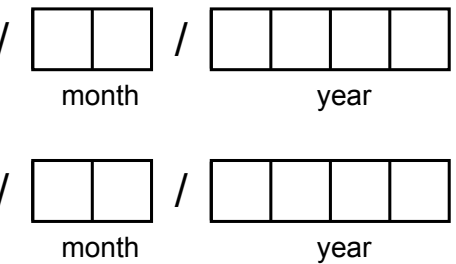

Female

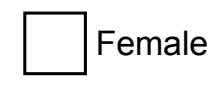

Patient's Gender: Male

Type of verruca (please cross all that apply) plantar calcaneous

plantar MTPJ

mosaic

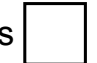

other

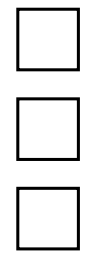

If other (please specify)

This patient was not eligible to take part in the trial because: (please cross all that apply)

The patient had a verruca, which could not be treated by either treatment.

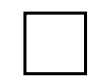

The patient was under 12 years of age.

The patient was unable to give informed consent.

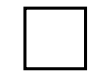

The patient had impaired healing eg due to diabetes, peripheral vascular disease or any other condition.

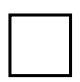


The patient was taking immunosuppressant drugs such as corticosteroids.

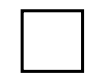

The patient was currently taking part in another trial evaluating other treatments for their verruca.

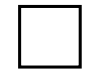

Other reason (Please specify)

THANK YOU FOR TAKING THE TIME TO COMPLETE THIS FORM.

PLEASE RETURN THIS FORM TO THE UNIVERSITY OF YORK IN THE PRE-PAID ENVELOPE PROVIDED.

https://www.hsytu.york.ac.uk/verruca/login.aspx 


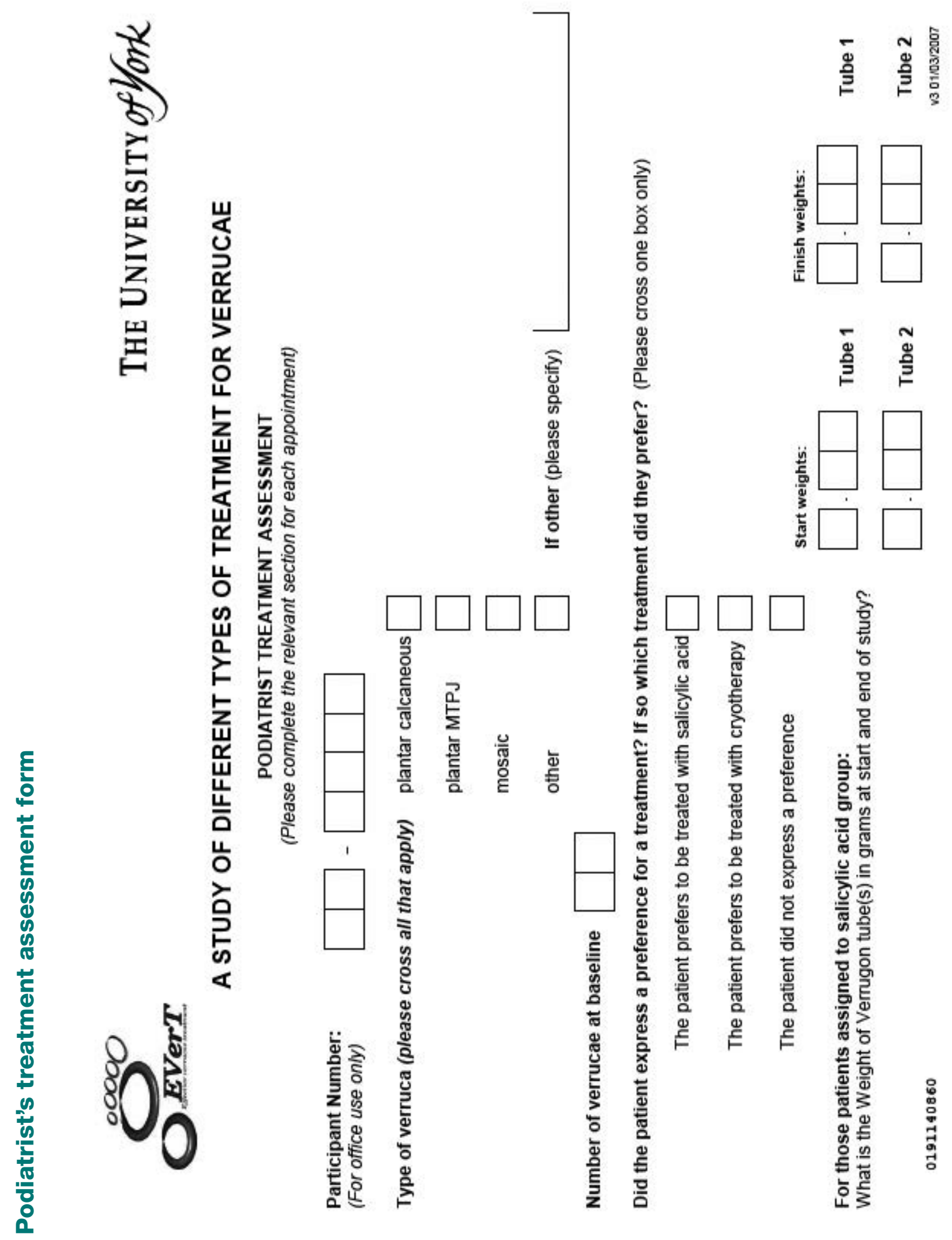




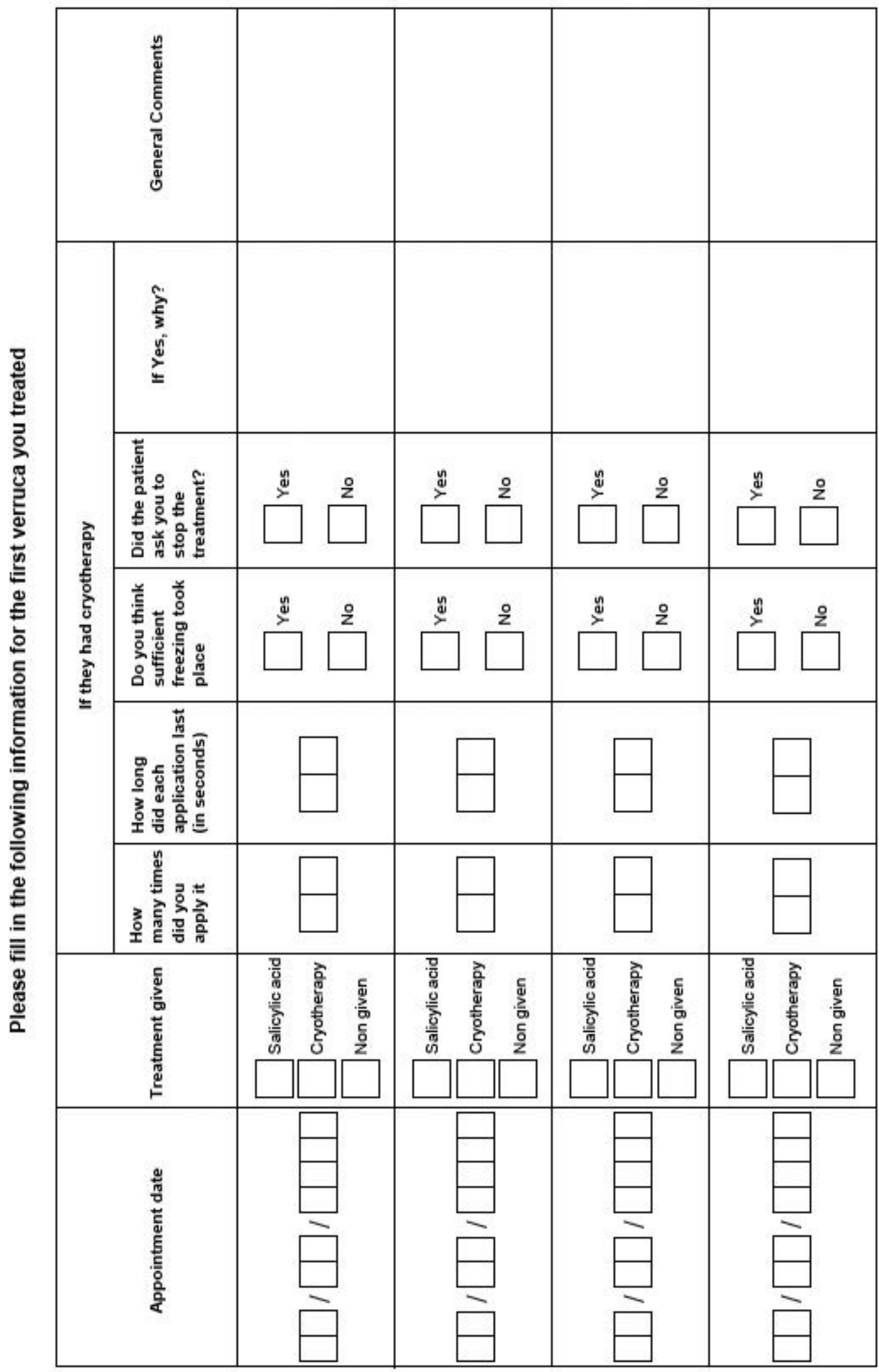

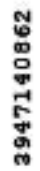




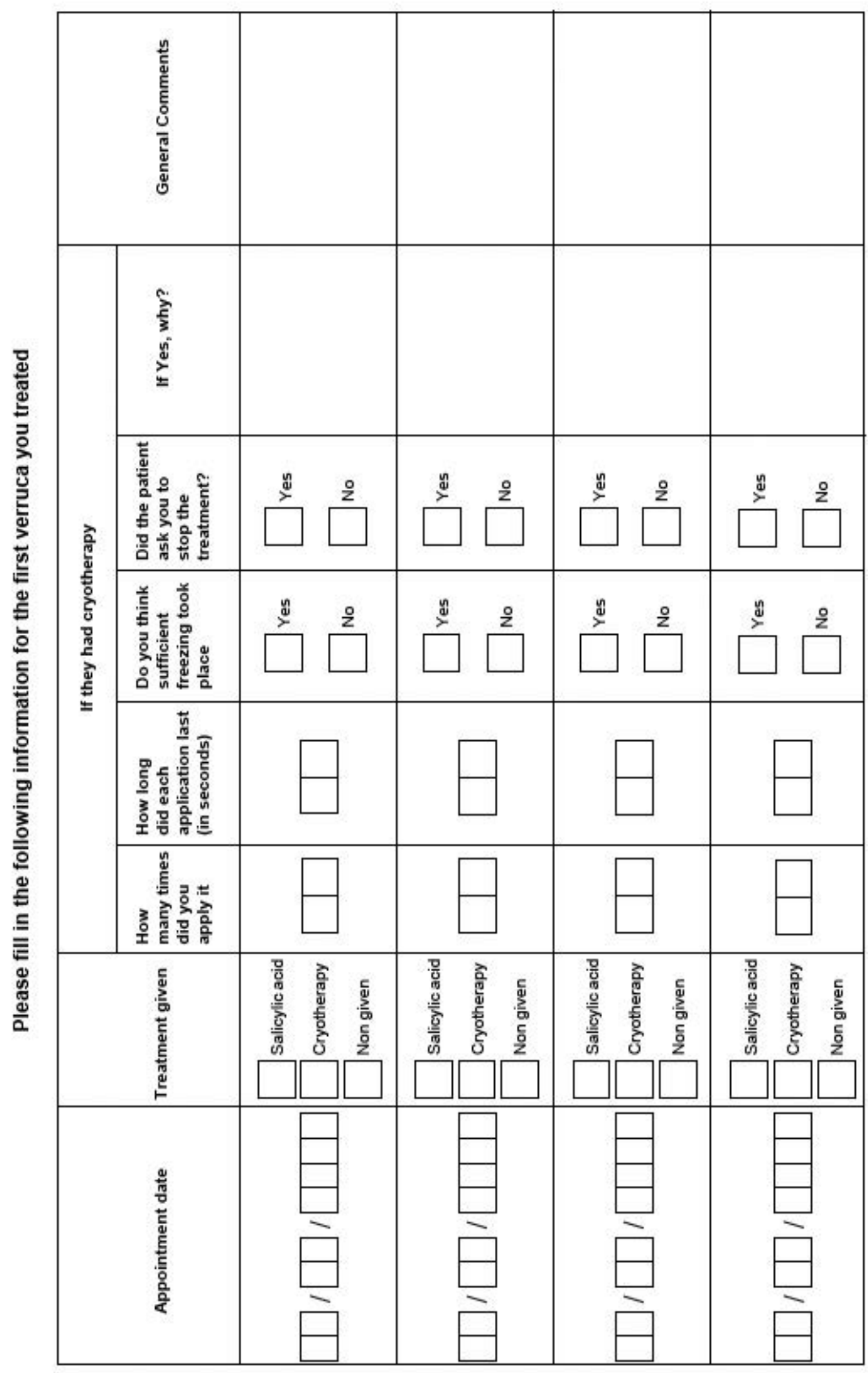

옹 


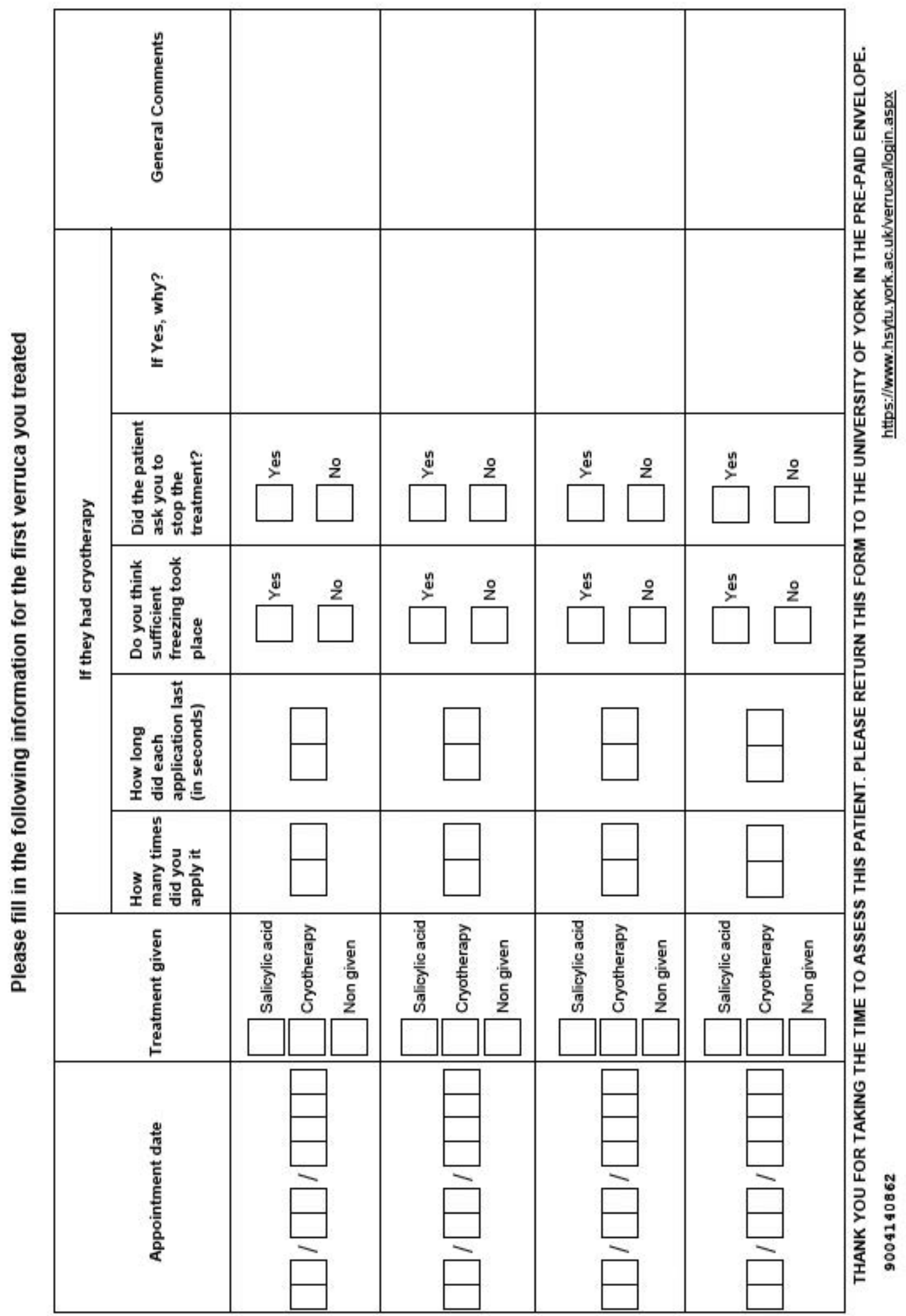


Patient pain questionnaire

\section{THE UNIVERSITY offlork}

\section{A STUDY OF DIFFERENT TYPES OF TREATMENT FOR VERRUCAE}

\section{PATIENT PAIN QUESTIONNAIRE}

Please complete this form immediately after your first treatment

\section{Participant Number:}

(For office use only)

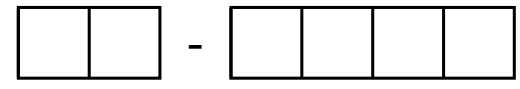

What is your date of birth?
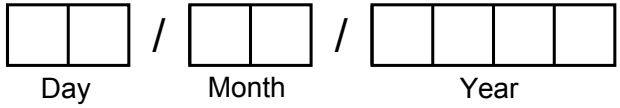

Are you?

Male

Female

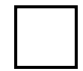

On a scale of 0 to 10 , how painful did you find your first treatment?

(where 0 is no pain and 10 is the worst pain imaginable)

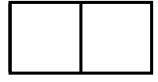

What is the date you are filling in this form?
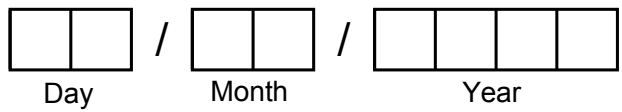

THANK YOU FOR TAKING THE TIME TO COMPLETE THIS QUESTIONNAIRE.

Please give it to the receptionist on your way out or return it to the York Trials Unit, University of York, Dept of Health Sciences, Area 4, Seebohm Rowntree Building, York Y010 5DD), in the prepaid envelope provided. 
Week-1 patient questionnaire

\section{THE University offlork}

\section{A STUDY OF DIFFERENT TYPES OF \\ TREATMENT FOR VERRUCAE}

FOLLOW-UP QUESTIONNAIRE WEEK 1

Participant Number:

(For office use only)

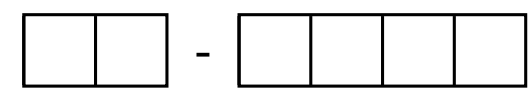




\section{PLEASE READ ALL THE INSTRUCTIONS BEFORE COMPLETING THE QUESTIONNAIRE}

Thank you for agreeing to take part in this evaluation.

Please answer ALL the questions. Although it may seem that questions are asked more than once, it is still important that you answer every one.

If you find it difficult to answer a question, do the best you can.

Please follow the instructions for each section carefully.

For each section, if you are asked to put a cross in the box, please use a cross rather than a tick, as if you were filling out a ballot paper.

For example in the following question, if your answer to the question is yes, you should place a cross firmly in the box next to yes.

Do you drive a car? Yes $\bigotimes$

No

If you are asked to circle a number, please use a circle rather than underlining a number.

For example, in the following question if you are asked 'how happy are you today?' where ' 1 ' is 'very unhappy' and ' 5 ' is 'very happy', if you feel neither happy nor unhappy you may wish to answer 3 . You do this by clearly circling the number 3.

Very

unhappy

2

\section{PLEASE USE A BLACK OR BLUE PEN.}

Please do not use a pencil or any other coloured pen.

Please read all the instructions for each section. 
Please enter the date you are completing this questionnaire:
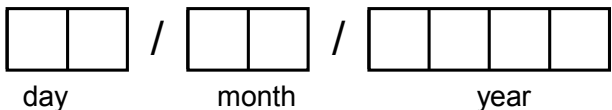

We would like to know your views about the treatment to your verruca:

1. How painful is your verruca today? (please circle one number only)
Not at all
A little bit
0
1
Moderately
2
Quite a lot
3
Extremely
4

2. If your verruca has been painful, have you found it necessary to take a pain killer?

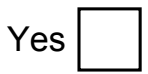

No

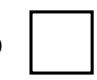

If 'yes' how many days did you find it necessary to take the pain killers due to your verruca treatment?

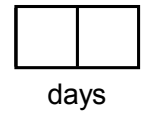

3. Have you had any other problems due to the verruca treatment? (Please specify)

4. How happy are you with your treatment? (please circle one number only)

$\begin{array}{ccccc}\begin{array}{c}\text { Very } \\ \text { unhappy }\end{array} & \text { Unhappy } & \begin{array}{c}\text { Neither } \\ \text { happy nor } \\ \text { unhappy }\end{array} & \text { Happy } & \begin{array}{c}\text { Very } \\ \text { happy }\end{array} \\ 1 & 2 & 3 & 4 & 5\end{array}$


5. If you have been asked to treat yourself at home with salicylic acid, how many times in the last 7 days have you applied it?

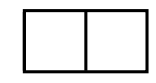

6. We would like to know about any other comments you may have about the treatment you are receiving for your verruca.

PLEASE RETURN THIS FORM TO THE UNIVERSITY OF YORK IN THE PRE-PAID ENVELOPE PROVIDED. THANK YOU FOR TAKING THE TIME TO COMPLETE THIS QUESTIONNAIRE.

https://www.hsytu.york.ac.uk/verruca/login.aspx 
Week-3 patient questionnaire

\section{THE UNIVERSITY of York}

\section{A STUDY OF DIFFERENT TYPES OF TREATMENT FOR VERRUCAE}

FOLLOW-UP QUESTIONNAIRE WEEK 3

Participant Number:

(For office use only)

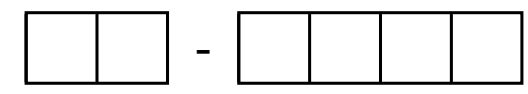


PLEASE READ ALL THE INSTRUCTIONS BEFORE COMPLETING THE QUESTIONNAIRE

Thank you for agreeing to take part in this evaluation.

Please answer ALL the questions. Although it may seem that questions are asked more than once, it is still important that you answer every one.

If you find it difficult to answer a question, do the best you can.

Please follow the instructions for each section carefully.

For each section, if you are asked to put a cross in the box, please use a cross rather than a tick, as if you were filling out a ballot paper.

For example in the following question, if your answer to the question is yes, you should place a cross firmly in the box next to yes.

Do you drive a car? Yes $\bigotimes$

No

If you are asked to circle a number, please use a circle rather than underlining a number.

For example, in the following question if you are asked 'how happy are you today?' where ' 1 ' is 'very unhappy' and ' 5 ' is 'very happy', if you feel neither happy nor unhappy you may wish to answer 3 . You do this by clearly circling the number 3.

Very

PLEASE USE A BLACK OR BLUE PEN.

Please do not use a pencil or any other coloured pen.

Please read all the instructions for each section. 
Please enter the date you are completing this questionnaire:
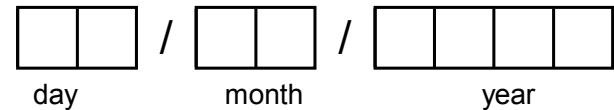

\section{SECTION 1}

We would like to know your views about the treatment to your verruca:

1. How painful is your verruca today? (please circle one number only)
Not at all
A little bit
Moderately
1
2
Quite a lot
0
3
Extremely
4

2. If your verruca has been painful, have you found it necessary to take a pain killer?

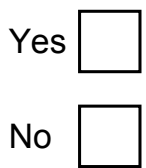

If 'yes' how many days did you find it necessary to take the pain killers due to your verruca treatment?

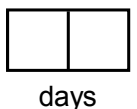

3. Have you had any other problems due to the verruca treatment? (Please specify)

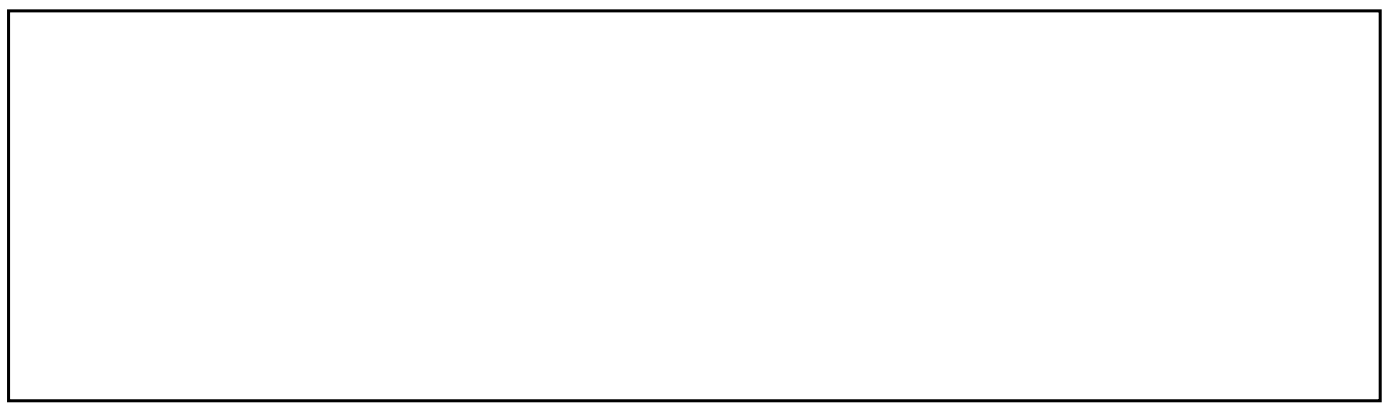

4. How happy are you with your treatment? (please circle one number only)

\begin{tabular}{|c|c|c|c|c|}
\hline $\begin{array}{l}\text { Very } \\
\text { unhappy }\end{array}$ & Unhappy & $\begin{array}{c}\text { Neither } \\
\text { happy nor } \\
\text { unhappy }\end{array}$ & Happy & $\begin{array}{c}\text { Very } \\
\text { happy }\end{array}$ \\
\hline 1 & 2 & 3 & 4 & 5 \\
\hline
\end{tabular}


5. If you have been asked to treat yourself at home with salicylic acid, how many times in the last 7 days have you applied it?

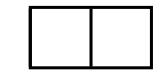

6. We would like to know about any other comments you may have about the treatment you are receiving for your verruca.

\section{SECTION 2}

This section asks about your verruca

1. Do you think your verruca has gone? (If you had more than one verrucae have they all gone?)

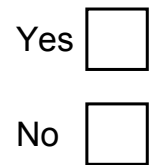

1a. If you answered 'Yes' to question 1, when did your verruca go? (If you had more than one verruca when did the last one go?)

Please state the date
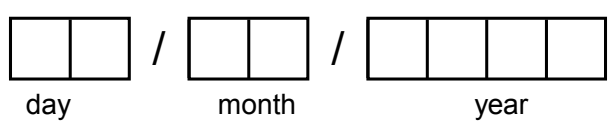

\section{PLEASE RETURN THIS FORM TO THE UNIVERSITY OF YORK IN THE PRE-PAID ENVELOPE PROVIDED. THANK YOU FOR TAKING THE TIME TO COMPLETE THIS QUESTIONNAIRE.}

https://www.hsytu.york.ac.uk/verruca/login.aspx 


\section{THE UNIVERSITY of York}

\section{A STUDY OF DIFFERENT TYPES OF TREATMENT FOR VERRUCAE}

Please complete this form when your verruca has gone.

Participant Number:

What is your date of birth?
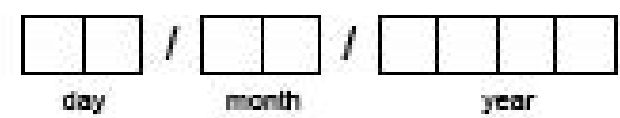

Please let us know the date your verruca disappeared.

My verruca went on
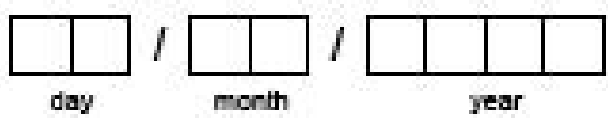

Please return this form to the York Trials Unit in the envelope provided or phone Sarah Cockayne at York University on 01904321736 or email esc5 פyork.ac.uk

Thank you.

https://umw.hsytu.york.ac.uk/vernuca/login.aspx 
Week-12 patient questionnaire

\section{THE UNIVERSITY of York}

\section{A STUDY OF DIFFERENT TYPES OF \\ TREATMENT FOR VERRUCAE}

FOLLOW-UP QUESTIONNAIRE WEEK 12

Participant Number:

(For office use only)

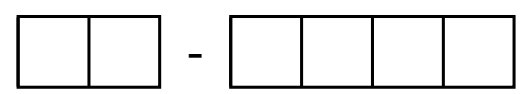




\section{PLEASE READ ALL THE INSTRUCTIONS BEFORE COMPLETING THE QUESTIONNAIRE}

Thank you for agreeing to take part in this evaluation.

Please answer ALL the questions. Although it may seem that questions are asked more than once, it is still important that you answer every one.

If you find it difficult to answer a question, do the best you can.

Please follow the instructions for each section carefully.

For each section, if you are asked to put a cross in the box, please use a cross rather than a tick, as if you were filling out a ballot paper.

For example in the following question, if your answer to the question is yes, you should place a cross firmly in the box next to yes.

Do you drive a car? Yes $\bigotimes$

No

If you are asked to circle a number, please use a circle rather than underlining a number.

For example, in the following question if you are asked 'how happy are you today?' where ' 1 ' is 'very unhappy' and ' 5 ' is 'very happy', if you feel neither happy nor unhappy you may wish to answer 3 . You do this by clearly circling the number 3.

Very

unhappy

1
2

PLEASE USE A BLACK OR BLUE PEN.

Please do not use a pencil or any other coloured pen.

Please read all the instructions for each section. 
Please enter the date you are completing this questionnaire:
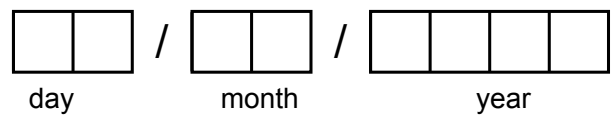

This section asks about your verruca:

1. Do you think your verruca has gone? (If you had more than one verrucae have they all gone?)

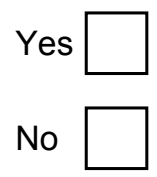

1a. If you answered 'Yes' to question 1, when did your verruca go? (If you had more than one verruca when did the last one go?)

Please state the date

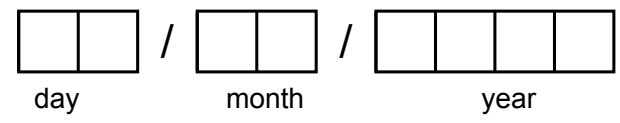

This section asks about the treatment you had for your verruca:

1. At the beginning of this study, you will have received treatment with either the acid paste or the freezing technique. During the study, did you find it necessary to stop the original treatment?

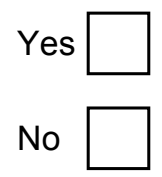

If 'Yes' what was your reason(s) for stopping the treatment?

1a. If you answered 'Yes' to question 1 in this section, did you start another treatment

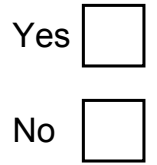

If 'Yes', please specify treatment 
2. Have you had any other problems due to the verruca treatment? (Please specify)

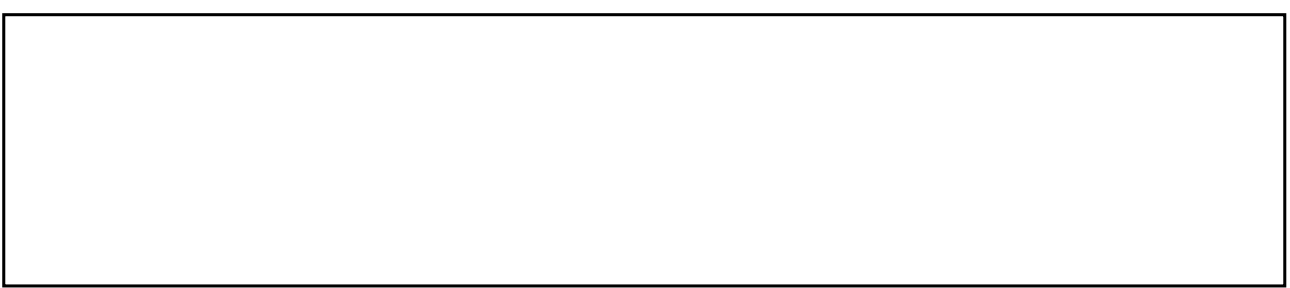

3. If you had another verruca, would you be willing to have the same treatment again?

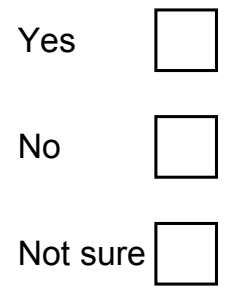

3a. Please could you tell us the reasons for your answer to question 3.

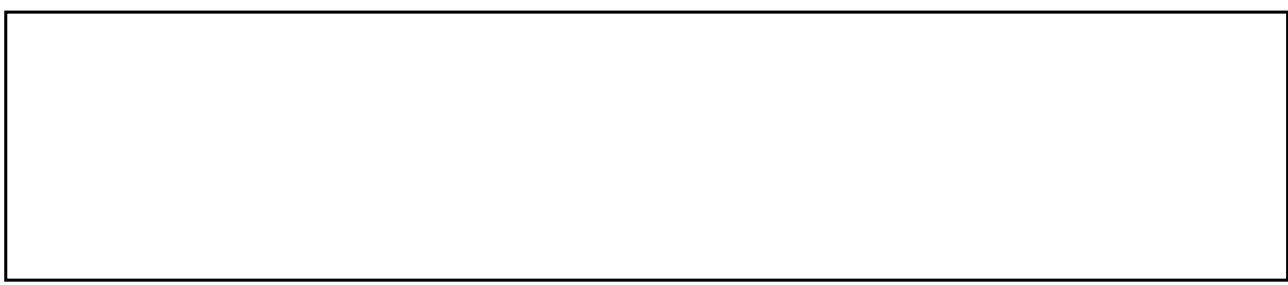

4. How happy are you with your treatment? (please circle one number only)

$\begin{array}{cccc}\begin{array}{c}\text { Very } \\ \text { unhappy }\end{array} & \text { Unhappy } & \begin{array}{c}\text { Neither } \\ \text { happy nor } \\ \text { unhappy }\end{array} & \text { Happy } \\ \text { happy }\end{array}$

1

3

4

5

\section{This section asks about the costs related to your treatment:}

1. How many visits in total did you make to the podiatry clinic for treatment to this verruca? (Please include the initial assessment, and all visits for treatment and redressings) 
2. During this course of treatment to your verruca, have you found it necessary to visit your General Practitioner or Practice Nurse regarding your verruca?

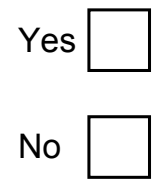

2a. If 'Yes' please state number of visits and date(s) of visit(s).

\section{General Practitioner}
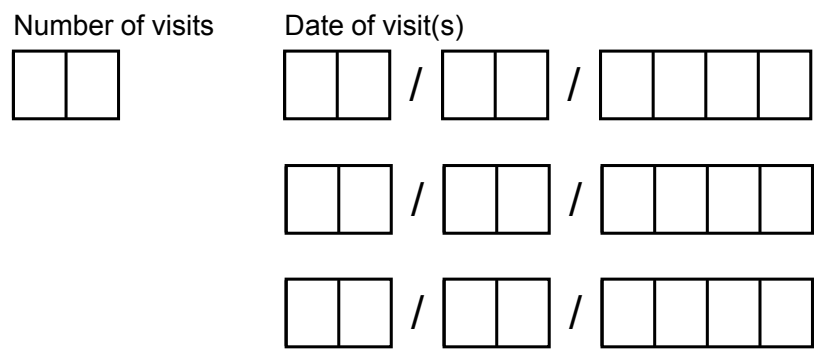

Practice Nurse
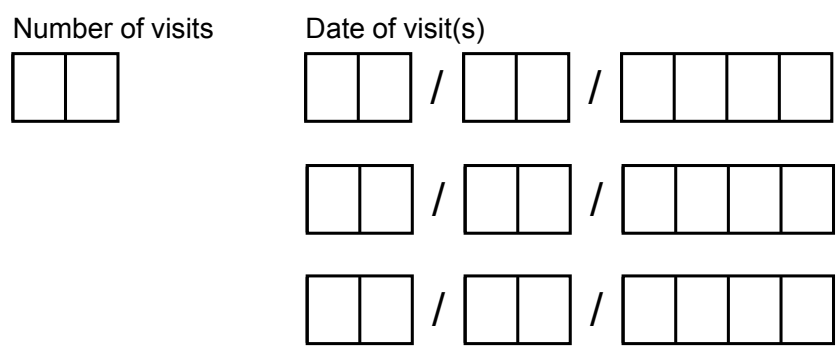

3. Have you had to see your GP for an emergency visit because of your verruca?

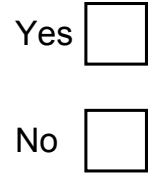

3a. If 'Yes' to question 3, please give details 
4. During this course of treatment to your verruca, have you purchased any verruca treatments yourself? (For example, treatments purchased over the counter)

Yes<smiles>C1CCC1</smiles>

No

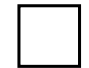

If 'Yes' can you tell us what you bought and how much it cost?

Type of treatment purchased

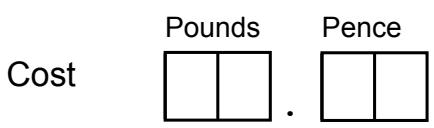

PLEASE RETURN THIS FORM TO THE UNIVERSITY OF YORK IN THE PRE-PAID ENVELOPE PROVIDED. THANK YOU FOR TAKING THE TIME TO COMPLETE THIS QUESTIONNAIRE. 
Verruca(e) has gone form

\section{The University of Jork}

\section{A STUDY OF DIFFERENT TYPES OF TREATMENT FOR VERRUCAE}

Please complete this form when your verruca has gone.

Participant Number:

What is your date of birth?
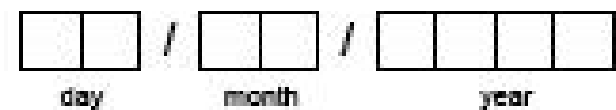

Please let us know the date your verruca disappeared.

My verruca went on

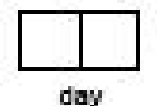

day
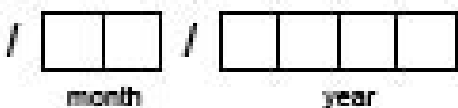

Please return this form to the York Trials Unit in the envelope provided or phone Sarah Cockayne at York University on 01904321736 or email esc5@york.ac.uk

Thank you. 


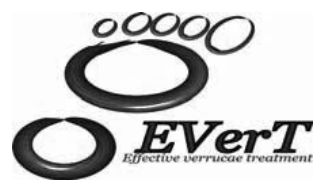

\section{THE UNIVERSITY of York}

\section{A STUDY OF DIFFERENT TYPES OF TREATMENT FOR VERRUCAE \\ PODIATRIST OUTCOME ASSESSMENT}

\section{Participant Number:}

(For office use only)

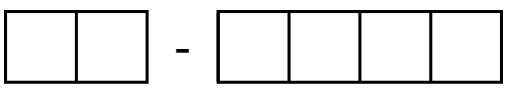

Please enter the date you are completing this questionnaire:
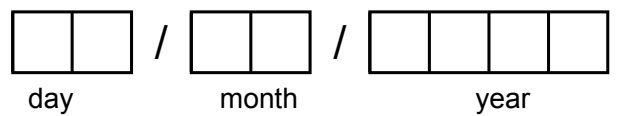

1. Does the verruca appear to be completely cleared/cured?

Yes

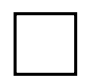

No

If 'No' how many verrucae are left?

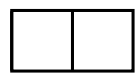

2. Did the patient require further treatment?

Yes

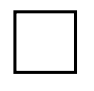

No

If 'Yes' what treatment did the patient receive?

Please specify

3. Any other comments? (Please specify)

PLEASE REMEMBER TO TAKE A DIGITAL PHOTOGRAPH.

THANK YOU FOR TAKING THE TIME TO ASSESS THIS PATIENT. PLEASE RETURN THIS FORM TO THE UNIVERSITY OF YORK IN THE PRE-PAID ENVELOPE PROVIDED.

https://www.hsytu.york.ac.uk/verruca/login.aspx 
Six-month patient questionnaire

\section{THE UNIVERSITY of York}

\section{A STUDY OF DIFFERENT TYPES OF \\ TREATMENT FOR VERRUCAE}

\section{SIX MONTH FOLLOW-UP QUESTIONNAIRE}

Participant Number:

(For office use only)

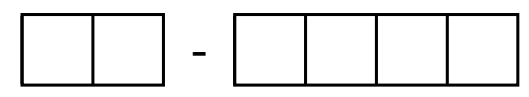

Date Sent:

(For office use only)
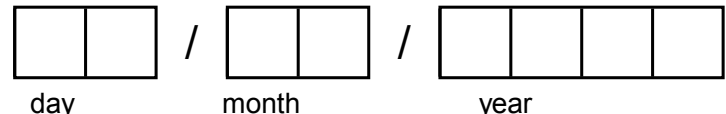

Participant's Date of Birth:
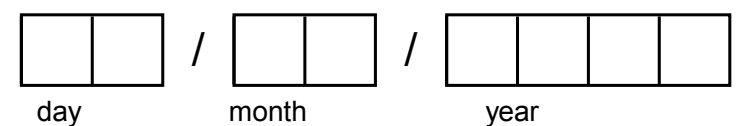


\section{PLEASE READ ALL THE INSTRUCTIONS BEFORE COMPLETING THE QUESTIONNAIRE}

Thank you for agreeing to take part in this evaluation.

Please answer ALL the questions. Although it may seem that questions are asked more than once, it is still important that you answer every one.

If you find it difficult to answer a question, do the best you can.

Please follow the instructions for each section carefully.

For each section, if you are asked to put a cross in the box, please use a cross rather than a tick, as if you were filling out a ballot paper.

For example in the following question, if your answer to the question is yes, you should place a cross firmly in the box next to yes.

Do you drive a car? Yes $\bigotimes$

No

If you are asked to circle a number, please use a circle rather than underlining a number.

For example, in the following question if you are asked 'how happy are you today?' where ' 1 ' is 'very unhappy' and ' 5 ' is 'very happy', if you feel neither happy nor unhappy you may wish to answer 3 . You do this by clearly circling the number 3.

Very

unhappy

1

2

(3)

4

Very happy

\section{PLEASE USE A BLACK OR BLUE PEN.}

Please do not use a pencil or any other coloured pen.

Please read all the instructions for each section. 
Please enter the date you are completing this questionnaire:

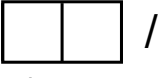

day

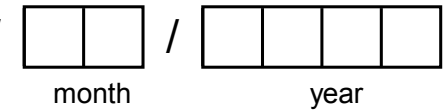

This section asks about your verruca:

1. Do you have any verruca(e) today? (Please cross one box only)

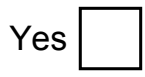

No

1a. If you answered 'No' to Question 1, when did your verruca go? (If you had more than one verruca when did the last one go?)

Please state the date

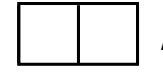

day

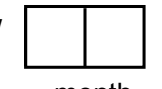

month

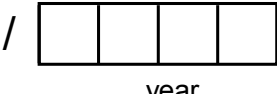

year

1b. If you answered 'Yes' to Question 1, where are they? (Please cross all that apply)

In the original place

In another place

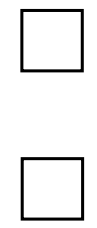

The following questions asks whether you have had further treatment for your verruca(e), please answer all that apply

2. If you still had a verruca(e) 12 weeks after you started the study, have you had any further treatment for it? (Please cross one box only)

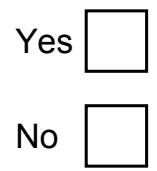

2a. If you answered 'Yes' to Question 2, was the treatment from the podiatrist/nurse/GP?

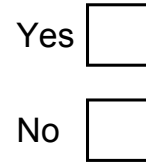

If Yes, please specify treatment received 


\section{And/OR}

2b. Have you purchased an over the counter treatment e.g. Bazuka, Wartner?

Yes

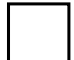

No

If Yes, please specify

\section{And/OR}

2c. Have you received another form of treatment for your verruca(e) e.g. homoeopathy?

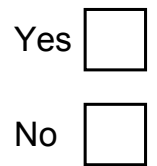

If Yes, please specify

3. We would like to know about any other comments you may have relating to your verruca

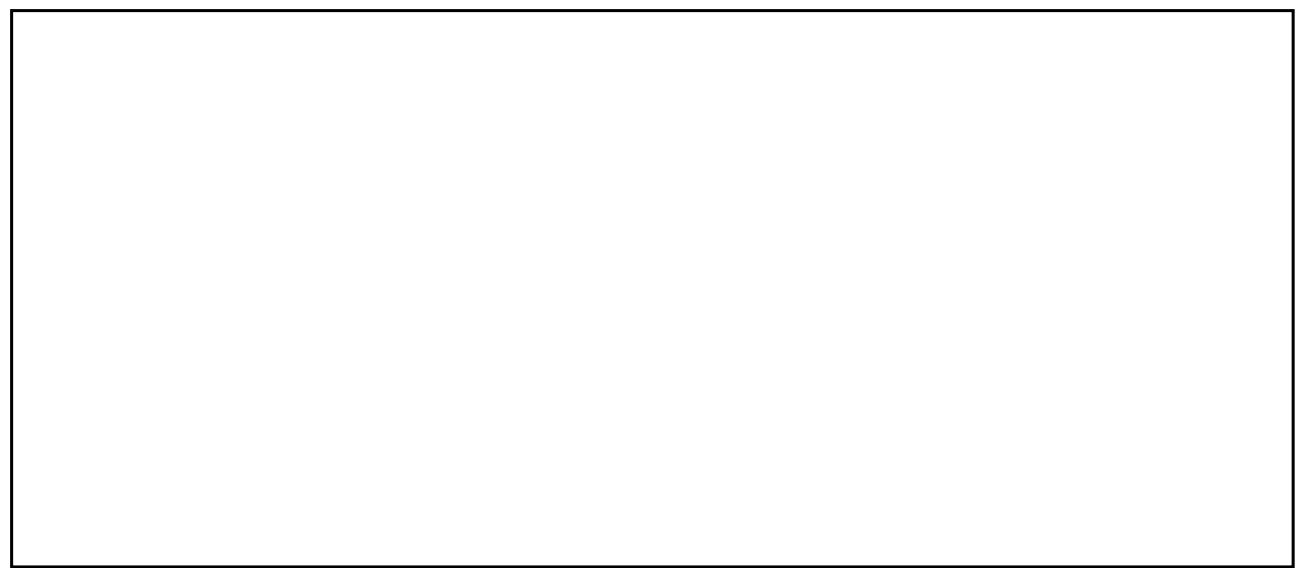

\section{PLEASE RETURN THIS FORM TO THE UNIVERSITY OF YORK IN THE PRE-PAID ENVELOPE} PROVIDED. THANK YOU FOR TAKING THE TIME TO COMPLETE THIS QUESTIONNAIRE.

Thank you for taking part in this study. The trial is due to end in Spring 2009. We will write to all participants to let them know the main results of the study.

https://www.hsytu.york.ac.uk/verruca/login.aspx 


\section{Change of circumstances form}

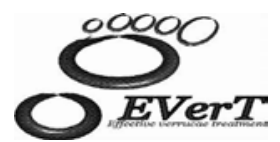

\section{THE UNIVERSITY of York}

\section{EVerT: A STUDY OF DIFFERENT TYPES OF TREATMENT FOR VERRUCAE Change of Circumstances Form}

Please complete this form if there are any changes in the circumstances of the EVerT participant.

Participant Trial Number:

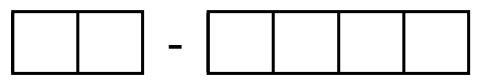

Please enter the date you are completing this questionnaire:
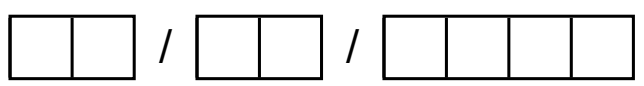

Reason for change in circumstance:

Please read the following and write the number of the MAIN reason in the box at the end of this form.

1. The patient no longer wishes to have the study treatment (Please state reason and new treatment if given)

L

2. The patient no longer wishes to complete postal questionnaires but agrees to follow up by the health care professional

3. The patient wishes to leave the study (Please state reason if given)

L

4. The patient is being withdrawn by podiatrist/nurse/doctor/other health care professional (Please state reason) L

5. The patient has died (please also complete a 'Serious Adverse Event Form')

Date of death:
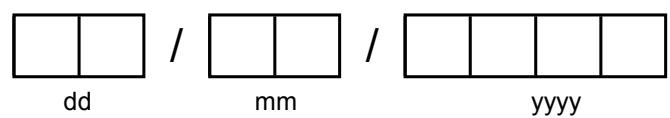

6. The patient is lost to follow up

7. Other reason (please state below)

The main reason for the change is option number

(Please write option number in box)

Please give more details, if applicable:

Please send this form to the York Trials Unit in the pre-paid envelope provided 
Non-serious adverse event form

\section{NON-SERIOUS ADVERSE EVENT FORM}

Patient concerned (trial number)

Name \& address of podiatrist reporting event.

Date of event

Details of event

Please include details of: site, signs, symptoms, severity, onset and duration of reaction, batch

number medicinal product, severity of event and any other information.

Action taken and outcome

Do you think the event is related to the trial treatment $(50 \%$ salicylic acid or cryotherapy using liquid nitrogen)? (Please tick only ONE box)
Unrelated
unlikely to
be related

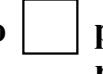
possibly related
probably related
definitely related instead.
- Death
- A life-threatening risk (that is an immediate risk of death)
- Hospitalisation of patient or prolongation of existing hospitalisation
- Persistent or significant disability/incapacity
- Consists of a congenital anomaly or birth defect

not able to

assess if

related

If the adverse event/reaction has resulted in any of the following you must complete a SAE form

Possible SAEs in the Verrucae trial. Please note that this is not an exhaustive list, if you suspect an event is serious, please contact the Trial co-ordinator-York Trials Unit. We would rather you erred on the side of caution and reported an event to us.

Suspected damage to underlying tissue eg tendon

Patient has died

Limb compromised:

Newly diagnosed diabetic: patient diagnosed as diabetic by GP during course of trial Patient hospitalised

Signature Date 
Review of non-serious adverse event form

\section{REVIEW OF NON-SERIOUS ADVERSE EVENT FORM}

$$
\bigodot_{\text {EverT }}^{\operatorname{cog} O}
$$

\section{REVIEW OF NON SERIOUS ADVERSE EVENT FORM}

Patient trial number

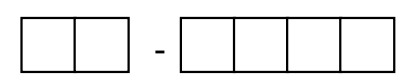

How and when notification of the event was made:

Date of review:

Action taken:

Signature of reviewer:

Date reviewed by DMC and Trial Steering Committee: 


\section{Serious adverse event form}

\section{SERIOUS ADVERSE EVENT/REACTION FORM EVerT Trial OEVerT}

\section{STUDY DETAILS:}

EVerT Cryotherapy versus salicylic acid for the treatment of verrucae.

EudraCT: 2004-000905-24 CTA: 22803/0001/001-0001 REC ref: 04/MRE04/59

\section{SUBJECT DETAILS:}

Patient's ID number

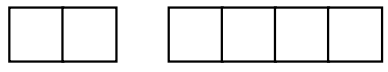

Patient's initials

Patient's date of birth

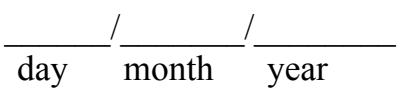
Male

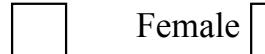

Patient's weight if known

Patient's height if known

\section{EVENT DETAILS:}

Date of onset of event

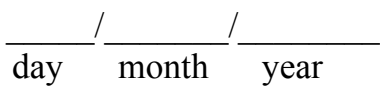

Description of event/reaction and action taken :

Classification of SAE: (Please tick all that apply)

Death

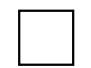

Life or limb threatening event

Hospitalisation required/prolonged

Persistent or significant disability/incapacity

Other medically important condition
Congenital anomaly or birth defect

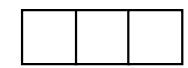

Female

Maximum intensity:

Mild $\square \quad$ Moderate $\square \quad$ Severe $\square$ 
OUTCOME of event at the time of this report:

(Tick one box only)

\section{Date Recovered/died}

Day month year

Recovered fully
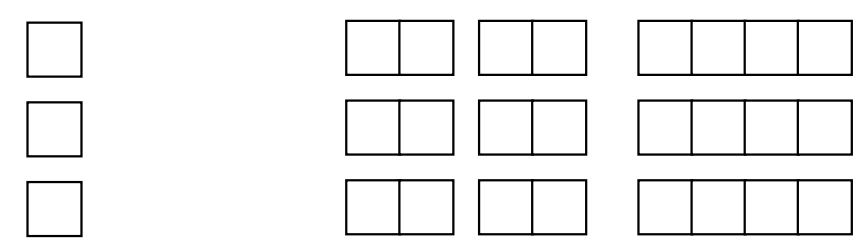

Recovered with sequelae

Died
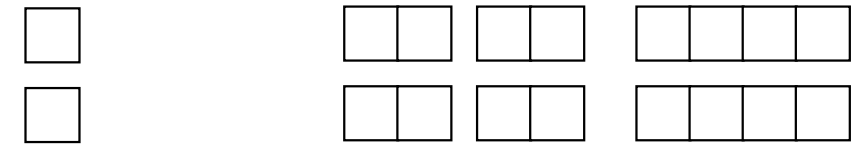

Ongoing

Relationship of event to treatment (tick one box only)

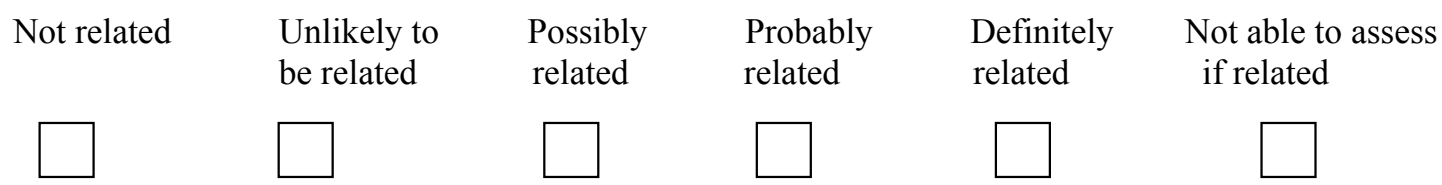

If possibly, probably or definitely related, was the SAE unexpected? (Unexpected means not described in the protocol or SMPC).'

Yes $^{1}$

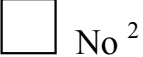

1 - The SAE is a SUSAR 2 - The SAE is not a SUSAR.

York Trials Unit must be notified of any serious adverse event by telephone (01904 321736) within 24 hours of onset of the event.

Post or fax top copy of this form and any available supporting documents to Sarah Cockayne, Trial Coordinator, Department of Health Sciences Area 4, Seebohm Rowntree Building, University of York, Heslington, York, YO10 5DD, within 48 hours of onset (Fax 01904 321387).

Please note that you may need to inform your Local Research Ethics Committee of this event.

\section{MEDICINAL PRODUCT DETAILS:}

Name of medicinal product (MP)

Batch number

Indication for which suspect investigational MP was prescribed

Dosage form and strength 
Daily dose and regiment (specify units)

Route of administration

Starting date and time of day of reaction

Date and time last dose given, or duration of treatment

Date of treatments

\section{CONCOMITANT MEDICATION:}

(Details of administration of other medication concurrent with the IMP

\section{DETAILS OF REPORTER OF EVENT:}

Name position and address of reporter of event:

Telephone number:

Email address:

Profession (Speciality)

Signature

Date

$$
\overline{\text { day }} \frac{/}{\text { month }}-\frac{}{\text { year }}
$$


Review of serious adverse events

\section{the University ofl/ork REVIEW OF SERIOUS ADVERSE EVENT/REACTION FORM}

Patient's trial number:

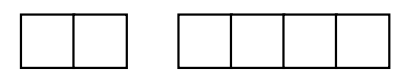

Date event reported to YTU:

$$
\text { Day } \frac{1}{\text { month }} \frac{1}{\text { year }}
$$

How notification of the event was made:

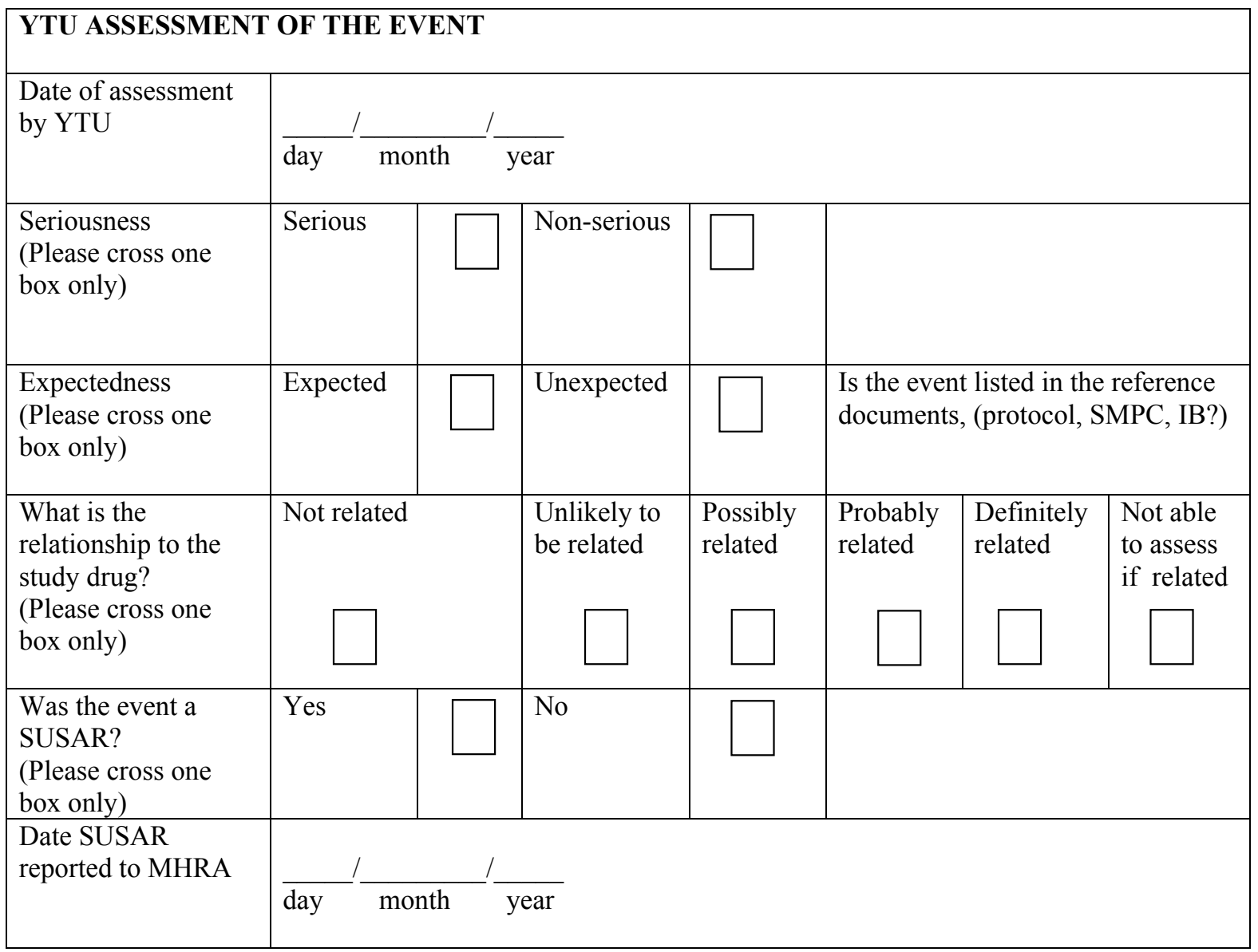




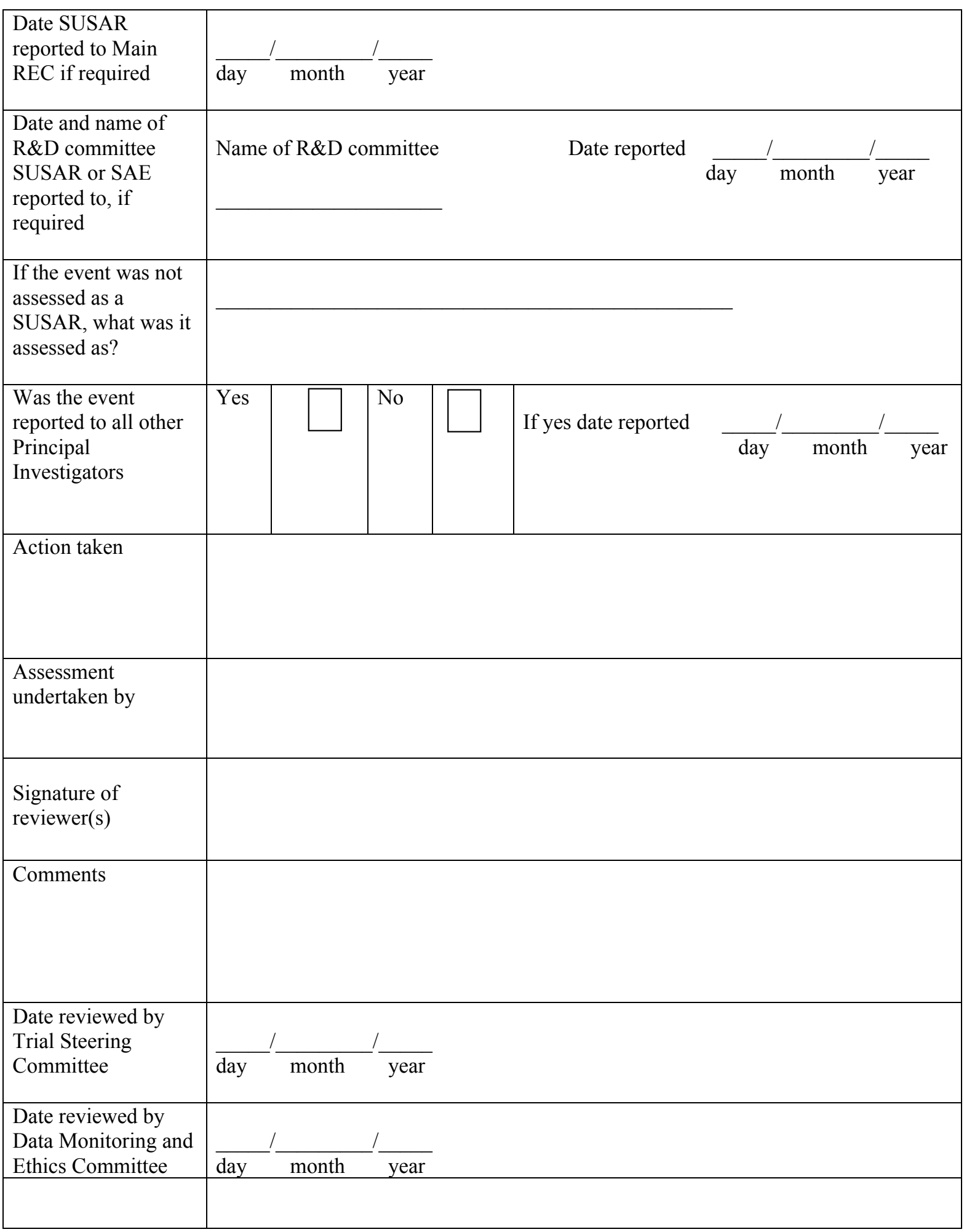





\section{Appendix 5}

\section{Advertising material}




\section{Poster - version 1}

\section{Helping Your Podiatrist, Nurse or GP Treat Your Verruca}

\section{Who can help?}

We are looking for 266 people with a verruca to take part in a research study. If you have a verruca, and are aged between 12 and 24 years of age, then you may be able to take part.

\section{What is the study about?}

Verrucae are a common problem. Many will disappear without treatment, however, patients may seek treatment if their verruca is painful or if they are prevented from doing sports. There are many different ways to treat verrucae, but it is unclear which treatment is best.

The purpose of this study is to compare two commonly used treatments to find out which of these is the best treatment to cure verrucae. We are also interested in your opinion of the treatments.

\section{What are the two treatments being} tested?

The two treatments being tested are an acid paste called Verrugon which you can buy over the counter from a pharmacist and a freezing technique which is applied by a Podiatrist, GP or Nurse.

\section{What will I have to do if I take part?}

The study will last for 6 months, but the treatments will last for a maximum of 8 weeks. If you are treated with the acid paste the Podiatrist, GP or Nurse will show you how to apply it at your first appointment. You will then be asked to take the treatment home with you and apply it daily up to a maximum of 8 weeks. If you are treated with the freezing technique, you will be treated by your Podiatrist, GP or Nurse at the clinic.

If you agree to take part, your Podiatrist, GP or nurse will record some details about your verruca and take a digital photograph of it. We will ask you to complete a brief questionnaire about your verrucae and during the 6 months of the study, we will send you a similar questionnaire after 1, 3, 12 , and 24 weeks.

\section{What should I do now?}

If you are interested in taking part in the study, please ring

(Insert local contact details)

and they will assess you in order to see if you are eligible for the study.

Alternatively, you can contact the Trial Manager Sarah Cockayne:

Tel 01904321736

or

Email: esc5@york.ac.uk

\section{0}

EVERT: A multi-centre randomised trial, funded by the NHS Health Technology Assessment Programme and

co-ordinated by the York Trials Unit at 
Poster - version 2

\section{DO YOU HAVEA VERROCAR}

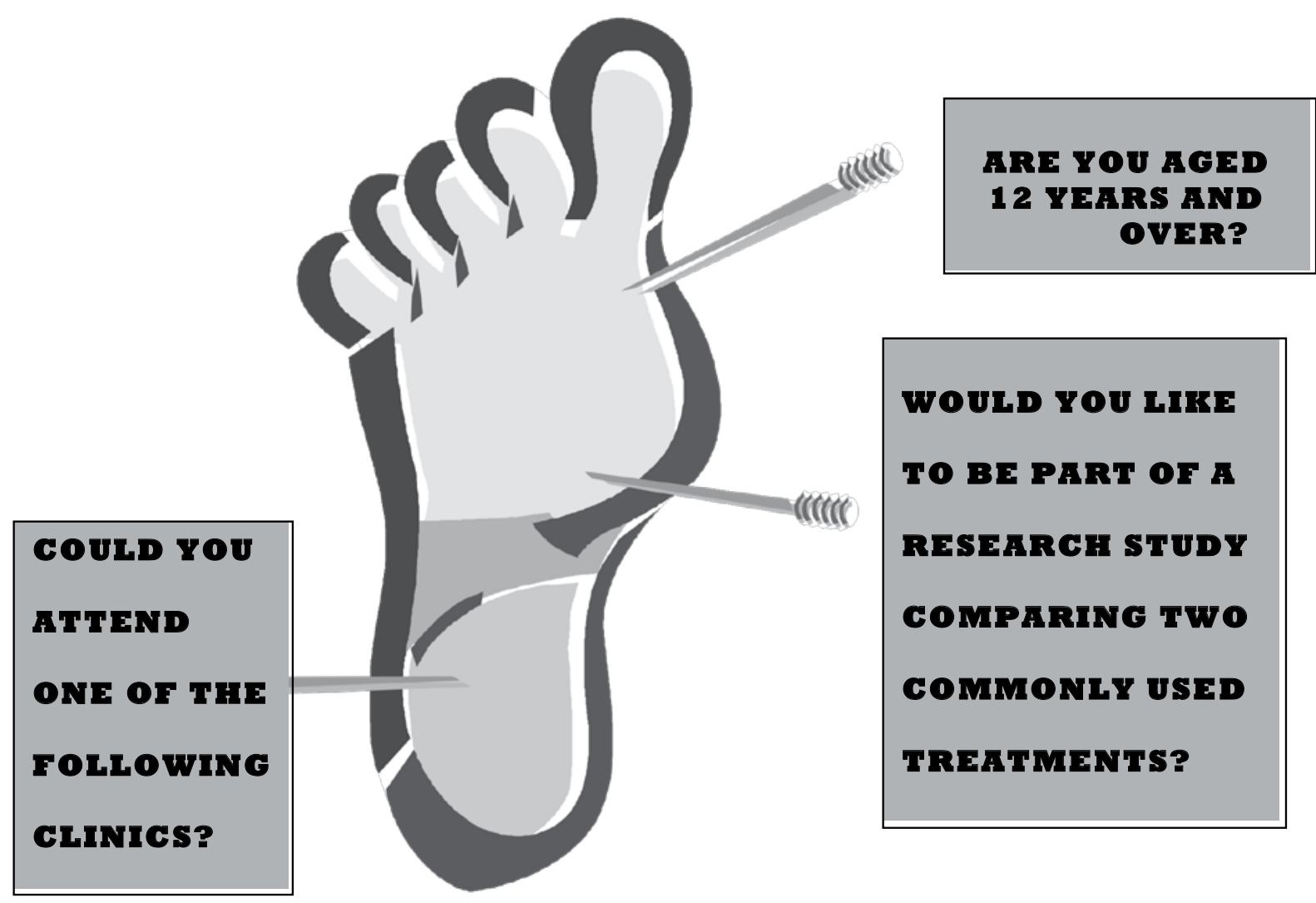

IF THE ANSWER IS 'YES' THEN WE WOULD LIKE TO HEAR FROM YOU!

Treatments will be provided free of charge and set travel expenses will be paid. For more information on how you could take part with no obligation please contact: Insert local contact details.

Version 2 


\title{
Generic press release
}

\section{(Insert logo of University/Trust)}

\section{Media Information}

\author{
DATE (insert date) \\ REFERENCE (insert reference)
}

\section{University puts Verrucae on trial}

Podiatry lecturer/Podiatrist/health care professional (name of podiatrist/health care professional) at (name of site) has announced the launch of a new clinical trial that will investigate solutions to the irritating and painful problem of verrucae.

The collaboration between (name of site) and the York Trials Unit at The University of York, UK will investigate which of two common treatments for verrucae - freezing with liquid nitrogen or an acid paste - is the most effective in terms of results and cost.

Patients volunteering to take part in the trial will be treated by qualified Podiatrists at (name of site), while researchers at The University of York will analyse the data.

Verrucae are a common viral infection and can be a painful problem. Although most will disappear eventually without treatment, patients often seek help if their verruca is painful or if they are prevented from doing sport.

There are many different ways to treat verrucae but it is unclear which treatment is best. Since verrucae are seen as a minor condition, few clinical trials have been funded into the best solutions for them.

(Name of podiatrist/health care professional) says: "We hope to study 266 patients in total and want to hear from people aged 12 years and over who have a verruca and are interested in taking part in the trial."

Patients will be asked to help for six months, but the treatments will only last for a maximum of eight weeks. Half of the patients will be asked to treat themselves daily with the acid paste, as directed by the (Podiatrist/health care professional), up to a maximum of eight weeks.

The other half will be treated with the freezing technique, applied by a (Podiatrist/health care professional), with re-treatment at follow-up appointments if required. Participants will also be asked about any side effects they experience and their satisfaction with their treatment.

(Name of podiatrist/health care professional) continues: "The information we get from this study should help us to treat future patients with verrucae more effectively." 


\section{If you are interested in taking part you should ring (insert clinic contact details and telephone number) you will be assessed in order to see if you are eligible for the study. The treatment provided will be free of charge.}

-ends-

\section{Notes for editors:}

- (Local note to editors about the university. For example for Northampton University: The University of Northampton is dedicated to high quality higher education at undergraduate and postgraduate levels, through taught courses and research. As Northamptonshire's only dedicated higher education institution, it is committed to the transfer of knowledge and technology to the community and aims to contribute to the cultural development of the region)

- This project is funded by the NHS R\&D Programme Health Technology Assessment (HTA) Programme (Project No. 05/513/02), and the results will be used to inform clinical practice within the NHS. The study has been reviewed and approved by Trent Multi Research Ethics Committee (UK). The views and opinions expressed herein are those of the authors and do not necessarily reflect those of the Department of Health (UK).

Details of press release officer 
SECOND PRESS RELEASE

(Insert logo of University/Trust)

Press Release

For immediate release

Media Information: Press Office (insert Press Office telephone number)

\section{Patients wanted to put best foot forward}

More patients are needed to join a clinical trial in the (name of town/city) area aimed at cutting the $£ 40$ million a year cost to the NHS of treating warts and verrucas.

Researchers at the University of York have been conducting the trial in (town/city) as part of a major study to establish the most effective solution for dealing with this irritating and painful problem.

The initial trial, aimed at establishing the most effective treatment for verrucas, was restricted to patients aged between 12 and 24, but now the study has been extended to include any patient aged over 12 years old.

Almost two million people see their GP about verrucas and warts each year, costing the NHS at least $£ 40$ million.

The York Trials Unit, in the University's Department of Health Sciences, has been working with health professionals in (name of town/city) to investigate which of two common treatments for verrucas - freezing with liquid nitrogen or an acid paste - is the most effective in terms of results and cost.

(Name of Trial co-ordinator/Principal Investigator) said: "The response from patients in (name of town/city) has been great, but now our funders, the Health Technology Assessment Programme, have agreed that we can extend the trial to any patient over the age of 12 . So we're looking for about 40 more patients from the (name of town/city) area to help us with the study. People interested in taking part should contact (name of podiatrist/health care professional) at (name of site) on (site telephone number)."

Patients will be asked to help for six months but the treatments will only last for a maximum of eight weeks. Half the patients will be asked to treat themselves daily with the acid paste, as directed, up to a maximum of eight weeks.

The other half will be treated with the freezing technique, applied by a podiatrist or health care professional, with re-treatment at follow-up appointments if required. Participants will be asked to fill in five questionnaires to find out about any side effects they experience and their satisfaction with their treatment

Volunteers will be assessed in order to see if they are eligible for the study and will need to attend the clinic for up to four additional appointments. Set travel expenses will be paid. The treatment provided will be free of charge.

\section{ENDS}


Notes for Editors:

- This project is funded by the NHS R\&D Programme Health Technology Assessment (HTA) Programme (Project No. 05/513/02), and the results will be used to inform clinical practice within the NHS. The study has been reviewed and approved by Trent Multi Research Ethics Committee. The views and opinions expressed herein are those of the authors and do not necessarily reflect those of the Department of Health.

- (University of York note may be included if appropriate: The Department of Health Sciences at the University of York is a large multi-disciplinary department, offering a broad range of taught and research programmes in the health care field, including nursing. It aims to develop the role of scientific evidence in health and health care through high quality research, teaching and other forms of dissemination).

- (If a recruiting site is situated in another university, an additional note about that university may be included. For example, for Northampton University: The University of Northampton is dedicated to high quality higher education at undergraduate and postgraduate levels, through taught courses and research. As Northamptonshire's only dedicated higher education institution, it is committed to the transfer of knowledge and technology to the community and aims to contribute to the cultural development of the region). 
SHORT PRESS RELEASE

logo of University/Trust)

\title{
Media Information
}

\author{
DATE (insert date) \\ REFERENCE (insert reference)
}

\section{(Name of University/Trust) puts Verrucae on trial}

(Name of Podiatry lecturer/Podiatrist/health care professional/Principal Investigator) at (name of site) is seeking patients to take part in a clinical trial that will investigate solutions to the irritating and painful problem of verrucae. (He/she) says "We want to hear from people aged 12 and over who have a verruca and are interested in taking part in the trial."

Patients will be asked to help for six months, but the treatments will only last for a maximum of eight weeks. Half of the patients will be asked to treat themselves daily with an acid paste, up to a maximum of eight weeks. The other half will be treated with a freezing technique, applied by a podiatrist/other healthcare professional, with re-treatment at follow-up appointments if required.

If you are interested in taking part you should ring (name of clinic/site) on telephone number (insert number) and ask for (contact name). You will be assessed in order to see if you are eligible for the study. The treatment provided will be free of charge.

This project is funded by the NHS R\&D Programme HTA Programme Project number 05/513/02. The views and opinions expressed therein are those of the authors and do not necessarily reflect those of the Department of Health. 


\section{Letters to pharmacists/GPs/parents of school students}

\section{THE UNIVERSITY of Fork}

(Pharmacist letter version 1 4/5/2007)
Insert Trust /site logo

DEPT OF HEALTH SCIENCES

University of York

Area 4, Seebohm Rowntree Building

York YO10 5DQ

Insert date

Name and address of pharmacist

Dear Name of pharmacist

\section{Re: A NHS HTA Programme funded trial of two treatments of verrucae}

We are writing to inform you about a research study which is currently being undertaken in your PCT. This study is being funded by the NHS Health Technology Assessment Programme under their call for Medicines for Children (http://www.hta.nhsweb.nhs.uk/calls/M4CUpdate.htm) and is a joint research project between the Podiatry Department at (insert name) and the York Trials Unit at the University of York. The aim of the study is to compare the clinical effectiveness of cryotherapy using liquid nitrogen versus patient daily self-treatment with an over the counter preparation of $50 \%$ salicylic acid (Verrugon) for the treatment of verruca.

This study aims to recruit 266 participants and we are writing to ask for your assistance with this study in two ways. First, we would like to ask you to put a poster advertising for trial participants in your pharmacy. Second, we would like you to inform suitable potential participants (ie individuals aged 12 to 24 years with a verruca) that the trial is being conducted, and if the patient agrees give them the contact details of the recruiting site (insert name and contact details of recruiting site). Participation in the trial will not involve any commitments on your part other than putting up a poster and passing on recruiting site contact details to potential participants.

As this study is being funded under a call for medicines for children, we currently wish to recruit participants aged between 12 to 24 years inclusively. Patients will however, be ineligible if they are currently in a trial evaluating other treatments for their verruca, are unable to give informed consent, have impaired healing eg due to diabetes, peripheral vascular disease, are immunosuppressed, are on renal dialysis, have cold intolerance or have any of the following conditions; blood dyscrasias of unknown origin, cryoglobulinaemia, cryofibrinogenaemia, collagen and auto-immune disease.

We have enclosed further details of the study with this letter, but if you require any additional information or would like to discuss the study further, please contact either (Name of Podiatrist/Health care professional) on (insert telephone number and email address) or (Name of trial coordinator and contact details).

Yours sincerely

Name of PI

Title
Professor David Torgerson Director of York Trials Unit
Sarah Cockayne

Trial coordinator 
Please contact Sarah Cockayne, telephone number 01904321736 or email esc5@york.ac.uk if you are able to put up a poster in your pharmacy. Alternatively please complete the form below and return it to Sarah Cockayne, in the pre-paid envelope provided.

Please send me a poster to put in our pharmacy:

Name of Pharmacist:

Address of Pharmacy

Telephone Number:

Email address: 
Title: Cryotherapy versus salicylic acid for the treatment of verrucae: A randomised controlled trial.

\section{Background}

Verrucae are a common, infectious and sometimes painful problem. Using incidence figures from the $4^{\text {th }}$ National Morbidity Survey (1991-92) ${ }^{\mathrm{i}}$ an unpublished economic decision model assessing the effectiveness and cost-effectiveness of salicylic acid and cryotherapy has estimated that almost 2 million people see their GP per year about cutaneous warts at a cost of at least $£ 40$ million per annum. Although most verrucae will spontaneously disappear without treatment many patients seek treatment to remove a verruca due to it being painful or because they are being prevented from doing sports.

A recent systematic review conducted by the Cochrane Skin Group assessed the effects of different local treatments of cutaneous, non-genital warts in healthy people. This review highlighted the uncertainty with respect to the optimal treatment of verrucae. There was however, some evidence from six trials to suggest that treatment with salicylic acid was more effective than placebo/no treatment, odds ratio 3.91 (95\% confidence interval 2.40 to 6.36 ). Freezing warts using cryotherapy is widespread. Many patients experience unpleasant side effects such as pain and blistering during cryotherapy treatment, yet the same review found no evidence to suggest that it is more effective than treatment with topical agents such as salicylic acid. Only two trials were identified which compared salicylic acid and/or lactic acid with cryotherapy, but there was no difference in the efficacy between the treatments (OR 1.15, 95\% CI 0.72 to 1.82). However, both trials were reported as low quality, due to unclear allocation concealment, inadequate blinding procedures, small sample sizes and inappropriate follow-up and analysis. There is a need therefore, for a high quality randomised controlled trial to ascertain which is the best approach for the treatment of verrucae.

\section{Primary objective}

To compare the clinical effectiveness of cryotherapy versus salicylic acid for the treatment of verrucae in terms of the complete clearance of all verrucae as observed on digital photographs taken at baseline and 12 weeks and assessed by an independent health care professional (eg podiatrist, GP, Practice nurse). Blinded health care professional assessment will be used if for some reason the digital photograph is not interpretable. 'Clearance' of verrucae will be defined as being the restoration of normal skin upon close inspection, as assessed by the health care professional.

\section{Secondary objectives}

To compare the cost effectiveness of cryotherapy versus salicylic acid for the treatment of plantar warts in terms of the complete clearance of all verrucae. To assess the acceptability of the two approaches and to investigate self-reported time to clearance of verruca and recurrence/clearance of verrucae at six months.

\section{Design}

The proposed study is a pragmatic, multicentre, randomised controlled trial (RCT) with equal randomisation. Patients with a verruca will be allocated equally between the two treatment groups, namely: 50\% salicylic acid paste and cryotherapy using liquid nitrogen. 


\section{Eligibility}

Inclusion criteria:

Patients will be included if

- The patient has a verruca that in the opinion of the health care professional is suitable for treatment with either salicylic acid or cryotherapy.

- And the patient is aged 12 years and over but under 25 years of age.

\section{Exclusion Criteria:}

Patients will be excluded if any of the following criteria apply:

- Are currently in a trial evaluating other treatments for their verruca

- Have impaired healing eg due to diabetes, peripheral vascular disease or any other condition which means the patient has impaired healing

- Patients that are immunosuppressed eg have agammaglobulinaemia, or are currently taking immunosuppressant drugs such as corticosteroids

- Are unable to give informed consent

- Are currently on renal dialysis

- Have cold intolerance eg Raynaud's syndrome or cold urticaria

- Have any of the following conditions - blood dyscrasias of unknown origin, cryoglobulinaemia, cryofibrinogenaemia, collagen and auto-immune disease

\section{Treatment details}

Participants will be randomised equally between the two arms: daily self-treatment by the patient with $50 \%$ salicylic acid; cryotherapy using liquid nitrogen delivered by the health care professional (for example podiatrist, practice nurse, General Practitioner).

\section{Daily self-treatment by the patient with $50 \%$ salicylic acid paste - Verrugon as per the manufacturer's instructions.}

- At the first appointment the health care professional will instruct the patient and/or their parent or guardian if appropriate, on its use. Thereafter, it will be applied daily for a maximum of 8 weeks as per the manufacturer's instructions as follows:

- The self-adhesive ring should be fixed with the hole over the verruca.

- Squeeze a little Verrugon ointment into the hole and directly onto the verruca.

- Remove backing paper from plaster.

- Cover ring completely with plaster. Seal into position.

- Repeat treatment daily after gently pumicing or filing off the dead part of the verruca.

\section{Treatment with cryotherapy using liquid nitrogen delivered by the health care professional.}

Callus surrounding the verrucae will first be debrided. The tissue surrounding the verruca will be prepared according to normal practice. The area will then be sprayed with liquid nitrogen to cover the verruca area totally. The health care professional should freeze the tissue until they are satisfied that the tissue has been frozen adequately (this will be about 10 seconds). $75 \%$ silver nitrate should NOT to be applied to site. If the health care professional believes the area surrounding the verruca 
should be padded after treatment, this will be done according to normal practice. The patient will be advised to keep the area dry for 24 hours and that the area maybe uncomfortable as the treatment removes infected skin by causing a blister. If the area is very painful the patient will be recommended to use the type of painkiller they would use for a headache, and as per the instructions on the packet. The health care professional will then re book for the next treatment 14 days later. On the patient's return the sequence should be repeated up to a maximum of four treatments.

\section{Primary outcome}

The primary outcome will be complete clearance of all verrucae as observed on digital photographs taken at baseline and 12 weeks and assessed by an independent health care professional. Blind health care professional assessment will also be assessed and will be used if for some reason the digital photograph is not interpretable. 'Clearance' of verrucae will be defined as being the restoration of normal skin upon close inspection, as assessed by the health care professional.

\section{Secondary outcomes}

Secondary outcomes are self-reported clearance of verrucae at six months, and self-reported time to clearance of verrucae. In addition to this side effects of treatment, pain intensity after treatment, use of painkillers, restrictions to lifestyle due to having the verruca and treatment details will be recorded and assessed by patient postal questionnaire.

\section{Sample size}

In this study we have decided to power the trial to show a $15 \%$ difference in effectiveness. We therefore, will recruit sufficient patients to give us $80 \%$ power ( $5 \%$ two sided significance) to show a difference in cure rates of $70 \%$ versus $85 \%$ at 12 weeks after treatment. This will require 120 patients in each group after allowing for a $10 \%$ drop-out rate we will require 133 in each treatment group (i.e. 266 in total).

The study has the necessary ethics, research and development and Medicines and HealthCare products Regulatory Agency approvals. 


\section{Letter to GPs (version 121 November 2006)}

\section{THE University of fork}

\author{
Insert Trust /site logo \\ DEPT OF HEALTH SCIENCES \\ University of York \\ Area 4, Seebohm Rowntree Building \\ York YO10 5DQ
}

Name and address of doctor/practice manager

Dear Name of doctor/practice manager

\section{Re: A NHS HTA Programme funded trial of two treatments of verrucae}

We are writing to inform you about a research study which is currently being undertaken in your PCT. This study is being funded by the NHS Health Technology Assessment Programme under their call for medicines for Children (http://www.hta.nhsweb.nhs.uk/calls/M4CUpdate.htm) and is a joint research project between the Podiatry Department at (insert name) and the York Trials Unit at the University of York. The aim of the study is to compare the clinical effectiveness of cryotherapy using liquid nitrogen versus patient daily self-treatment with an over the counter preparation of $50 \%$ salicylic acid (Verrugon) for the treatment of verruca.

This study aims to recruit 266 participants and we are writing to ask for your assistance with this study in two ways. First, we would like to ask you to consider referring suitable patients who present to you for treatment of a verruca, to the podiatry department for treatment. Second to put a poster advertising for trial participants in the waiting room at your surgery. Participation in the trial should not involve any financial or clinical commitments on your part. The only time commitment involved will be in writing the referral letter to the podiatry clinic.

As this study is being funded under a call for medicines for children, we currently wish to recruit participants aged between 12 to 24 years inclusively. Patients will however, be ineligible if they are currently in a trial evaluating other treatments for their verruca, are unable to give informed consent, have impaired healing eg due to diabetes, peripheral vascular disease, are immunosuppressed, are on renal dialysis, have cold intolerance or have any of the following conditions; blood dyscrasias of unknown origin, cryoglobulinaemia, cryofibrinogenaemia, collagen and auto-immune disease.

We have enclosed further details of the study with this letter, but if you require any additional information or would like to discuss the study further, please contact either (Name of Podiatrist/Health care professional) on (insert telephone number and email address) or insert details of trial coordinator.

Yours sincerely

Name of CI

Title
Professor David Torgerson

Director of York Trials Unit
Sarah Cockayne

Trial coordinator 
Please contact Sarah Cockayne, telephone number 01904321736 or email esc5@york.ac.uk if you are able to put up a poster in your surgery. Alternatively please complete the from below and return it to Sarah Cockayne, in the pre-paid envelope provided.

Please send me a poster to put in our waiting room to:

Name of GP practice:

Practice Manager:

Practice Address:

Telephone Number:

Email address: 


\section{Title: Cryotherapy versus salicylic acid for the treatment of verrucae: A randomised controlled trial.}

\section{Background}

Verrucae are a common, infectious and sometimes painful problem. Using incidence figures from the $4^{\text {th }}$ National Morbidity Survey (1991-92 $)^{\mathrm{iii}}$ an unpublished economic decision model assessing the effectiveness and cost-effectiveness of salicylic acid and cryotherapy has estimated that almost 2 million people see their GP per year about cutaneous warts at a cost of at least $£ 40$ million per annum. Although most verrucae will spontaneously disappear without treatment many patients seek treatment to remove a verruca due to it being painful or because they are being prevented from doing sports.

A recent systematic review conducted by the Cochrane Skin Group assessed the effects of different local treatments of cutaneous, non-genital warts in healthy people ${ }^{\text {iv }}$. This review highlighted the uncertainty with respect to the optimal treatment of verrucae. There was however, some evidence from six trials to suggest that treatment with salicylic acid was more effective than placebo/no treatment, odds ratio 3.91 (95\% confidence interval 2.40 to 6.36). Freezing warts using cryotherapy is widespread. Many patients experience unpleasant side effects such as pain and blistering during cryotherapy treatment, yet the same review found no evidence to suggest that it is more effective than treatment with topical agents such as salicylic acid. Only two trials were identified which compared salicylic acid and/or lactic acid with cryotherapy, but there was no difference in the efficacy between the treatments (OR $1.15,95 \%$ CI 0.72 to 1.82 ). However, both trials were reported as low quality, due to unclear allocation concealment, inadequate blinding procedures, small sample sizes and inappropriate follow-up and analysis. There is a need therefore, for a high quality randomised controlled trial to ascertain which is the best approach for the treatment of verrucae.

\section{Primary objective}

To compare the clinical effectiveness of cryotherapy versus salicylic acid for the treatment of verrucae in terms of the complete clearance of all verrucae as observed on digital photographs taken at baseline and 12 weeks and assessed by an independent health care professional (eg podiatrist, GP, Practice nurse). Blinded health care professional assessment will be used if for some reason the digital photograph is not interpretable. 'Clearance' of verrucae will be defined as being the restoration of normal skin upon close inspection, as assessed by the health care professional.

\section{Secondary objectives}

To compare the cost effectiveness of cryotherapy versus salicylic acid for the treatment of plantar warts in terms of the complete clearance of all verrucae. To assess the acceptability of the two approaches and to investigate self-reported time to clearance of verruca and recurrence/clearance of verrucae at six months.

\section{Design}

The proposed study is a pragmatic, multicentre, randomised controlled trial (RCT) with equal randomisation. Patients with verrucae will be allocated equally between the two treatment groups, namely: $50 \%$ salicylic acid paste and cryotherapy using liquid nitrogen. 


\section{Eligibility}

Inclusion criteria:

Patients will be included if

- The patient has a verruca that in the opinion of the health care professional is suitable for treatment with either salicylic acid or cryotherapy.

- And the patient is aged 12 years and over but under 25 years of age.

\section{Exclusion Criteria:}

Patients will be excluded if any of the following criteria apply:

- Are currently in a trial evaluating other treatments for their verruca

- Have impaired healing eg due to diabetes, peripheral vascular disease or any other condition which means the patient has impaired healing

- Patients that are immunosuppressed eg have agammaglobulinaemia, or are currently taking immunosuppressant drugs such as corticosteroids

- Are unable to give informed consent

- Are currently on renal dialysis

- Have cold intolerance eg Raynaud's syndrome or cold urticaria

- Have any of the following conditions - blood dyscrasias of unknown origin, cryoglobulinaemia, cryofibrinogenaemia, collagen and auto-immune disease

\section{Treatment details}

Participants will be randomised equally between the two arms: daily self-treatment by the patient with $50 \%$ salicylic acid; cryotherapy using liquid nitrogen delivered by the health care professional (for example podiatrist, practice nurse, General Practitioner).

\section{Daily self-treatment by the patient with $50 \%$ salicylic acid paste - Verrugon as per the manufacturer's instructions.}

- At the first appointment the health care professional will instruct the patient and/or their parent or guardian if appropriate, on its use. Thereafter, it will be applied daily for a maximum of 8 weeks as per the manufacturer's instructions as follows:

- The self-adhesive ring should be fixed with the hole over the verruca.

- Squeeze a little Verrugon ointment into the hole and directly onto the verruca.

- Remove backing paper from plaster.

- Cover ring completely with plaster. Seal into position.

- Repeat treatment daily after gently pumicing or filing off the dead part of the verruca.

\section{Treatment with cryotherapy using liquid nitrogen delivered by the health care professional.}

Callus surrounding the verrucae will first be debrided. The tissue surrounding the verruca will be prepared according to normal practice. The area will then be sprayed with liquid nitrogen to cover the verruca area totally. The health care professional should freeze the tissue until they are satisfied that the tissue has been frozen adequately (this will be about 10 
seconds). $75 \%$ silver nitrate should NOT to be applied to site. If the health care professional believes the area surrounding the verruca

should be padded after treatment, this will be done according to normal practice. The patient will be advised to keep the area dry for 24 hours and that the area maybe uncomfortable as the treatment removes infected skin by causing a blister. If the area is very painful the patient will be recommended to use the type of painkiller they would use for a headache, and as per the instructions on the packet. The health care professional will then re book for the next treatment 14 days later. On the patient's return the sequence should be repeated up to a maximum of four treatments.

\section{Primary outcome}

The primary outcome will be complete clearance of all verrucae as observed on digital photographs taken at baseline and 12 weeks and assessed by an independent health care professional. Blind health care professional assessment will also be assessed and will be used if for some reason the digital photograph is not interpretable. 'Clearance' of verrucae will be defined as being the restoration of normal skin upon close inspection, as assessed by the health care professional.

\section{Secondary outcomes}

Secondary outcomes are self-reported clearance of verrucae at six months, and self-reported time to clearance of verrucae. In addition to this side effects of treatment, pain intensity after treatment, use of painkillers, restrictions to lifestyle due to having the verruca and treatment details will be recorded and assessed by patient postal questionnaire.

\section{Sample size}

In this study we have decided to power the trial to show a $15 \%$ difference in effectiveness. We therefore, will recruit sufficient patients to give us $80 \%$ power $(5 \%$ two sided significance) to show a difference in cure rates of $70 \%$ versus $85 \%$ at 12 weeks after treatment. This will require 120 patients in each group after allowing for a $10 \%$ drop-out rate we will require 133 in each treatment group (i.e. 266 in total).

The study has the necessary ethics, research and development and Medicines and HealthCare products Regulatory Agency approvals. 


\section{Letter to parent of school children}

Insert local logo

\section{THE UNIVERSITY of fork}

Insert contact details of local site

Department of Health Sciences

University of York

Area 4, Seebohm Rowntree Building

York YO10 5DQ

Date

Dear Parent

\section{Re: Patients needed for a trial of treatments for verrucas}

We are writing to ask if you would like to help us in a research project about verrucas, which is being funded by the NHS. This is a joint research project between the (insert name of site) and the York Trials Unit at the University of York.

Verrucas are a common problem and there are several different ways to treat them. This aim of this study is to compare two of these treatments to find out which treatment is better. We will be comparing a freezing technique, which is applied by a (podiatrist/doctor/nurse) and an acid paste (Verrugon), which you apply at home.

If your child has a verruca then they may be able to take part and help our research. For more information about the study, please see the leaflet overleaf or email (Name of local PI) or Sarah (address below).

Yours faithfully

$\begin{array}{lll}\text { Name of PI } & \text { Professor David Torgerson } & \text { Mrs Sarah Cockayne } \\ \text { Post } & \text { Director York Trials Unit } & \text { Trial coordinator, York Trials Unit } \\ \text { Email: (insert details) } & & \text { Email: esc5@york.ac.uk }\end{array}$





\section{Appendix 6}

\section{Flow chart for EVerT trial}

Potential participants identified by the health-care professional (HCP) are sent an appointment, patient information sheet, baseline questionnaire and consent form

\begin{tabular}{|c|c|c|}
\hline \multicolumn{2}{|c|}{$\begin{array}{l}\text { Participants who wish to take part in the study give written informed } \\
\text { consent and complete the patient baseline questionnaire. HCP } \\
\text { completes baseline data on podiatrist treatment assessment form, } \\
\text { randomisation form and takes digital photograph }\end{array}$} & $\begin{array}{l}\text { Participants who do not wish to } \\
\text { take part in the trial or are } \\
\text { ineligible revert to normal care }\end{array}$ \\
\hline \multicolumn{3}{|c|}{$\begin{array}{l}\text { HCP telephones YTU randomisation service/uses online web system to randomise the participant. HCP records } \\
\text { group allocation on randomisation form. HCP sends patient baseline questionnaire and photograph to YTU }\end{array}$} \\
\hline \multicolumn{3}{|c|}{ What treatment has the patient been allocated to? } \\
\hline Participant daily self-treatment with $50 \%$ salicylic acid & \multicolumn{2}{|c|}{ Cryotherapy using liquid nitrogen } \\
\hline $\begin{array}{l}\text { Participant given } 50 \% \text { salicylic acid, additional } \\
\text { plasters/felt pads and advice about how to apply } \\
\text { the ointment. Patient given advice sheet to take } \\
\text { home and patient pain questionnaire to } \\
\text { complete at home and return to YTU } \\
\text { by Freepost. HCP completes podiatrist } \\
\text { treatment assessment form and e-mails } \\
\text { photograph to YTU }\end{array}$ & $\begin{array}{r}\text { HCP deb } \\
\text { per no } \\
\text { lesion } \mathrm{i} \\
\text { is padded } \\
\text { sheet to } \\
\text { to compl } \\
\mathrm{HCP} \text { CC }\end{array}$ & $\begin{array}{l}\text { ounding callus and masks area as } \\
\text { ice. Applies liquid nitrogen until } \\
\text { ely frozen. The surrounding area } \\
\text { rmal practice. Patient given advice } \\
\text { le and patient pain questionnaire } \\
\text { ne and return to YTU by Freepost. } \\
\text { oodiatrist treatment assessment } \\
\text {-mails photograph to YTU }\end{array}$ \\
\hline \multicolumn{3}{|c|}{$\begin{array}{l}\text { Participant's GP is sent notification of their participation in the study. The participant is sent follow-up } \\
\text { questionnaires at } 1,3 \text { and } 12 \text { weeks and } 6 \text { months after entry into the study to return to YTU by Freepost }\end{array}$} \\
\hline $\begin{array}{l}\text { Participant seen } 2 \text { weeks post randomisation for } \\
\text { safety check. Is given additional Verrugon tubes } \\
\text { and felt pads/plasters if required. HCP completes } \\
\text { podiatrist treatment assessment form and takes } \\
\text { digital photograph and e-mails to YTU }\end{array}$ & $\begin{array}{r}\text { Particip } \\
\text { treatme } \\
\text { podiatr } \\
\text { digital }\end{array}$ & $\begin{array}{l}\text { es up to three more cryotherapy } \\
\text {-week intervals. HCP completes } \\
\text { ent assessment form and takes } \\
\text { h at each visit and e-mails them } \\
\text { to the YTU }\end{array}$ \\
\hline $\begin{array}{l}\text { All participants seen at week } 12 \text { for blinded outcom } \\
\text { assessment form. HCP takes a digital photograp } \\
\text { assessment form and outcome a }\end{array}$ & $\begin{array}{l}\text { sessment. } \\
\text { d e-mails } t \\
\text { ssment forr }\end{array}$ & $\begin{array}{l}\text { completes podiatrist outcome } \\
\text { sends podiatrist treatment } \\
\text { by Freepost }\end{array}$ \\
\hline
\end{tabular}





\section{Appendix 7}

\section{Trial protocol}




\title{
THE UNIVERSITY of York
}

PROTOCOL FOR: Cryotherapy versus salicylic acid for the treatment of verrucae: A randomised controlled trial.

Protocol version 10

\author{
16 Oct 2008
}

Funded by the HTA

Prof David Torgerson

Prof Ian Watt

Principal Investigator 


\section{AMENDMENTS TO PROTOCOL}

\section{A Protocol Version 229 September 2004}

Change in concentration of salicylic acid from $60 \%$ to $50 \%$ Verrugon.

\section{B Protocol version $32^{\text {th }}$ July 2006}

In light of the changes made due to obtaining funding from the HTA and after discussions held with the Trial Management Team the following changes were made:

- Additional background information added

- Clarification of primary and secondary outcomes

- Additional exclusion criteria added

- Clarification of treatment details for both salicylic acid and cryotherapy

- Additional recruitment strategies included

- Addition of web-based randomisation service added

- Notification of participant's GP involvement in the study included

- Clarification of non recruitment and use of 'Ineligible Patient Form'

- Clarification of ethical arrangements, reporting and monitoring adverse events and obtaining informed consent

- Additional section on treatment of missing data

\section{Protocol version $421^{\text {st }}$ November 2006}

In light of the advice from the Trial Steering Committee held on the $20^{\text {th }}$ September 2006 and discussions held with the trial management team it was decided to

- Clarify the secondary outcomes and the economic analysis

- Add additional exclusion criteria

- Clarify treatment details for patients presenting with more than one verrucae and the regimen for cryotherapy treatment

- The influence of prognostic variables on the primary outcome will be also be investigated.

\section{Protocol version 5 4/5/2007}

In light of the advice from the Trial Steering Committee held on the 22 March 2007 and discussions held with the trial management team it was decided to:

- Clarify the exclusion criteria to read 'oral' corticosteroids, not corticosteroids

- Further clarification to cryotherapy regimen: debridement prior to treatment now no longer necessary for the trial but if carried out should be done as per normal practice eg scalpel, file and a record kept of the method used; method of application of liquid 
nitrogen is changed to normal practice eg spray probe or cotton bud if there is a choice then spray should be used; time interval between treatments to $2 / 3$ weeks as there is no evidence to suggest there is a significant difference in effectiveness in treatments 2 or 3 weeks apart, and no further benefit from treating more than 4 times.

- We will ask participants who did not attend their outcome assessment appointment at 12 weeks if they would be able and willing to take a digital photo of their foot/feet and send it to the York Trials Unit EverT email account

- Clarification that data on adverse events will be collected by patient self-report

- Minor clarification to the economic analysis (patient perspective was missed out)

- Minor clarification to the reporting of adverse events

\section{E Protocol version 6 July 2007}

- Clarification that Professor David Torgerson is the Chief Investigator and Prof Ian Watt is Co-Chief Investigator.

- Additional exclusion criteria of patients with neuropathy

- Additional information about the storage and dispensing of Verrugon and supply of liquid nitrogen

- Adverse event/reaction reporting. Additional information included about reporting time of adverse events/reactions, duration of reporting, out of hours contact

F Protocol version $75^{\text {th }}$ September 2007

- Additional sites of Camden and Sefton PCT, Brownlow and Springfield Practice added.

G Protocol version $82^{\text {nd }}$ November 2007

- Additional sites included: Islington PCT and Dr Mittal

H Protocol version 920 February 2008

- Additional site included: Sheffield PCT and additional recruitment strategies, clarification of treatment of missing data

\section{Protocol version 1016 Oct 2008}

- Space for signature of Principal Investigator added to front page

- Amended to read Verrugon is to be applied 'once' daily rather than daily

- A sample of the Verrugon label is included 
- Clarification that patients should see their out of hours GP if a problem occurs outside of normal working hours

- Participants who attend their 12 week outcome assessment to be sent $£ 20$ to cover any expenses incurred

- Patients to be sent an unconditional $£ 5$ with the 12 week questionnaire, to cover expenses incurred when completing questionnaires.

- Clarified wording to read "If a clinician feels that the potential participant is unable to give informed consent, then they would not be eligible to take part in the study."

- Clarified wording to read "This sample size will also enable us to show that"

- Clarified to read "giving a total of 270 participants"

- Camden and Sefton PCT removed from list of sites. Claughton Medical Centre (Birkenhead, Wirral), Woodplumpton Road Surgery (Fulwood, Preston) and Arlington Road Surgery (Eastbourne) added as new sites.

- Amended Project Timetable to reflect approved extension of project 


\section{Key Contacts}

\section{Professor Ian Watt, Co-Chief Investigator}

Professor of Primary \& Community Care

Department of Health Sciences

Seebohm Rowntree Building (Area 2)

York YO10 5DD

Tel (01904) 321341

Email ian.watt@york.ac.uk

Professor David Torgerson, Co-Chief Investigator Health Economist

York Trials Unit, University of York,

Seebohm Rowntree Building (Area 4)

York YO10 5DD

Tel: 01904321306

Email david.torgerson@york.ac.uk

Mrs Sarah Cockayne, and trial co-ordinator

York Trials Unit, University of York,

Seebohm Rowntree Building (Area 4)

York YO10 5DD

Tel: 01904321736

Emailsarah.cockayne@york.ac.uk

Dr Mike Curran, Podiatrist

Senior Lecturer in Podiatry

School of Health

University of Northampton

Boughton Green Road

Northampton NN2 7AL

Tel: 01604892771

Emailmike.curran@northamptom.ac.uk

Dr Farina Hashmi, Podiatrist

Research Fellow

School of Health, Sport and Rehabilitation Sciences

Centre for School of Health, Sport and Rehabilitation Sciences Research Frederick Road Campus

University of Salford

Salford

M6 6PU

Tel: 01612953514

Email: F.Hashmi@salford.ac.uk

Dr Kim Thomas, Associate Professor in Dermatology Clinical Trial Centre of Evidence Based Dermatology

King's Meadow Campus

University of Nottingham, NG7 2NR

Tel 01158468632

Email Kim.Thomas@nottingham.ac.uk 
Dr Nichola McLarnon, Podiatrist

Lecturer in Podiatry

Glasgow Caledonian University

School of Health and Social Care

70 Cowcaddens Road,

Glasgow G4 0BA

Tel 01413313227

Email n.mclarnon@gcal.ac.uk

Ms Veronica Morton, Trial Statistician

York Trials Unit, University of York,

Seebohm Rowntree Building (Area 4)

York YO10 5DD

Tel: 01904321365

Emailveronica.dale@york.ac.uk

\section{Mr Ben Cross, Data Manager}

York Trials Unit, University of York,

Seebohm Rowntree Building (Area 4)

York YO10 5DD

Tel: 01904321364

Email ben.cross@york.ac.uk 


\section{BACKGROUND}

Verrucae or plantar warts are a common, infectious and sometimes painful problem. Using incidence figures from the $4^{\text {th }}$ National Morbidity Survey (1991-92) ${ }^{\mathrm{i}}$ an unpublished economic decision model assessing the effectiveness and cost-effectiveness of salicylic acid and cryotherapy has estimated that almost 2 million people see their GP per year about cutaneous warts at a cost of at least $£ 40$ million per annum. Although most verrucae will spontaneously disappear without treatment many patients seek treatment to remove a verruca due to it being painful or because they are being prevented from doing sports.

A recent systematic review conducted by the Cochrane Skin Group assessed the effects of different local treatments of cutaneous, non-genital warts in healthy people ${ }^{\mathrm{ii}}$. This review highlighted the uncertainty with respect to the optimal treatment of verrucae. There was however, some evidence from six trials to suggest that treatment with salicylic acid was more effective than placebo/no treatment, odds ratio 3.91 (95\% confidence interval 2.40 to 6.36). Freezing warts using cryotherapy is widespread (an unpublished survey of GP practices in Nottingham found that $71 \%$ offered cryotherapy for the treatment of warts in 2002 - Thomas, personal communication). Many patients experience unpleasant side effects such as pain and blistering during cryotherapy treatment, yet the same review found no evidence to suggest that it is more effective than treatment with topical agents such as salicylic acid. Only two trials were identified which compared salicylic acid and/or lactic acid with cryotherapy, but there was no difference in the efficacy between the treatments (OR 1.15, 95\% CI 0.72 to 1.82). However, both trials were reported as low quality, due to unclear allocation concealment, inadequate blinding procedures, small sample sizes and inappropriate follow-up and analysis.

Since verrucae are seen as a 'minor' condition few trials have been funded in this area. In addition to this of the 52 trials included in the systematic review only 3 were classed as high quality and $75 \%$ were classified as low quality. There is a need therefore, for a high quality randomised controlled trial with a cost effectiveness analysis to ascertain which is the best approach for the treatment of plantar warts. 


\section{RESEARCH OBJECTIVES}

\section{Primary objective}

To compare the clinical effectiveness of cryotherapy versus salicylic acid for the treatment of plantar warts in terms of the complete clearance of all verrucae as observed on digital photographs taken at baseline and 12 weeks and assessed by an independent health care professional (eg podiatrist, GP, Practice nurse). Blinded health care professional assessment will be used if for some reason the digital photograph is not interpretable. 'Clearance' of verrucae will be defined as being the restoration of normal skin upon close inspection, as assessed by the health care professional.

\subsection{Secondary objectives}

To compare the cost effectiveness of cryotherapy versus salicylic acid for the treatment of plantar warts in terms of the complete clearance of all verrucae. To assess the acceptability of the two approaches and to investigate

- Self-reported time to clearance of verrucae

- Recurrence/clearance of verrucae at six months

In addition to this, side effects of treatment, pain intensity after treatment, use of painkillers, restrictions to lifestyle due to having the verruca, treatment details will be recorded. Patient satisfaction with the treatment and the number of verrucae will also be recorded. 


\section{DESIGN}

The proposed study is a pragmatic, multicentre, randomised controlled trial (RCT) with equal randomisation. Patients with a verruca will be allocated equally between the two treatment groups, namely: 50\% salicylic acid paste and cryotherapy using liquid nitrogen.

\section{ELIGIBILITY}

\subsection{Inclusion Criteria}

Patients will be eligible if all of the following criteria apply:

- The patient has a verruca that in the opinion of the health care professional is suitable for treatment with both salicylic acid and cryotherapy.

- Are aged 12 years and over

\subsection{Exclusion Criteria}

Patients will be excluded if any of the following criteria apply:

- Are currently in a trial evaluating other treatments for their verruca

- Have impaired healing eg due to diabetes, peripheral vascular disease or any other condition which means the patient has impaired healing

- Patients that are immunosuppressed eg have agammaglobulinaemia, or are currently taking immunosuppressant drugs such as oral_corticosteroids

- Are unable to give informed consent

- Are currently on renal dialysis

- Have cold intolerance eg Raynaud's syndrome or cold urticaria

- Have any of the following conditions - blood dyscrasias of unknown origin, cryoglobulinaemia, cryofibrinogenaemia, collagen and auto-immune disease

- Patients with neuropathy

\section{TREATMENT DETAILS}

Participants will be randomised equally between the two arms: daily self-treatment by the patient with 50\% salicylic acid; cryotherapy using liquid nitrogen delivered by the health care professional (for example podiatrist, practice nurse, General Practitioner). If a patient 
presents with more than one verruca, then the Health Care Professional should treat the verrucae as they would in normal practice.

A 'no treatment' arm will not be included in this study. There are a number of reasons for this. Firstly, the systematic review showed that salicylic acid is more effective than "no treatment', whilst failing to find any evidence for the effectiveness of cryotherapy. Therefore, the important clinical question is whether the use of cryotherapy is superior to that of the standard effective treatment. Secondly, a 'no treatment' arm may lead to bias due to resentful demoralisation, particularly in those patients where the verrucae are painful, longstanding and resistant to previous treatment.

\subsection{Daily self-treatment by the patient with $\mathbf{5 0 \%}$ salicylic acid paste - Verrugon}

- At the first appointment the health care professional will instruct the patient and/or their parent or guardian if appropriate, on its use. Thereafter, it will be applied once daily for a maximum of 8 weeks as per the manufacturer's instructions as follows:

- The self-adhesive ring should be fixed with the hole over the verruca.

- Squeeze a little Verrugon ointment into the hole and directly onto the verruca.

- Remove backing paper from plaster.

- Cover ring completely with plaster. Seal into position.

- Repeat treatment daily after gently pumicing or filing off the dead part of the verruca

Patients will be asked to return all the Verrugon tubes they have received during the duration of the trial to the health care professional at their 12 week appointment. The health care professional will then weigh the tube to determine how much Verrugon has been used over the 8 week period.

Only those patients enrolled in this study may receive the Verrugon. The 50\% salicylic acid will be provided by the sponsor and will be dispatched to the treatment centre to be dispensed to participants by either a podiatrist, doctor nurse prescriber, pharmacist, other qualified health care practitioner or under a patient group directive. Drug accountability is a responsibility of study site personnel and overall drug accountability records will be kept to provide information on stock, dispensing and drug returns. 
The salicylic acid should be labelled as per the labelling requirements for investigational medicinal products used in clinical trials which come under requirements of Directive 2001/20/EC and the Medicines for Human Use (Clinical Trials) Regulations 2004 which implement the Directive and came into force on the 1 May 2004. A sample of the labelling is given here:

Verrugon ointment 50\% salicylic acid.
Trial EUDRAct number 2004-000905-24
Investigator: Name of investigator
Directions for use: as directed by the instructions give by the manufacturers.
Patient Name
Patient ID
Batch Number $\quad$ Expiry date $\quad$ Date of dispensing
Name and address of podiatry school/podiatry clinic supplier
Keep out of reach of children $\quad$ For clinical trials use only

Verrugon should be stored in a secure area, out of direct sunlight and below 25 degrees centigrade. All unused study drug, including undispensed supplies and supplies returned by the patient will be retained until the end of the study.

\subsection{Treatment with cryotherapy using liquid nitrogen delivered by the health care professional}

Although not necessary for the trial, sometimes it is the normal practice to debride prior to treatment with liquid nitrogen. If this is the case then the callus surrounding the verrucae will be debrided according to normal practice (eg with a scalpel, file, or not debrided at all) with any haemorrhages stopped by digital pressure only. The tissue surrounding the verruca will be prepared according to normal practice (eg unmasked, or masked with, for example vaseline). Liquid nitrogen will then be applied according to normal practice (eg spray, probe or cotton bud). If there is a choice in the method of application, then a spray should be used. The health care professional should freeze the tissue until they are satisfied that the tissue has 
been frozen adequately (this will be about 10 seconds if using a spray). $75 \%$ silver nitrate should NOT to be applied to site. If the health care professional believes the area surrounding the verruca should be padded after treatment, this will be done according to normal practice eg padded with $7 \mathrm{~mm}$ felt cavitied padding. The patient will be advised to keep the area dry for 24 hours and that the area maybe uncomfortable as the treatment removes infected skin by causing a blister. If the area is very painful the patient will be recommended to use the type of painkiller they would use for a headache, and as per instruction on the packet. The health care professional will then re book for the next treatment 14 or 21 days later. On the patient's return the sequence should be repeated up to a maximum of four treatments.

The recruiting site will use the equipment and liquid nitrogen used in normal practice to deliver cryotherapy treatment or will under prior agreement with the York trials unit be provided with the equipment. Storage of liquid nitrogen should comply with the current health and safety guidelines.

It is anticipated that some patients will request cryotherapy to be stopped. If the health care professional is not satisfied sufficient freezing has taken place then Verrugon will be offered as the second line treatment. Treatment details for both groups will be collected via questionnaire from the health care professional carrying out the treatment along with an assessment of how painful the treatment was (on a scale of 0 to 10) at the first visit. All participants will be given a follow-up appointment at 12 weeks to assess whether the treatment had been successful or not.

If required, patients in both groups will be able to book a 'fast-track' appointment to see their health care professional if they are concerned about adverse reactions to the treatment. If the patient has a problem with their verruca treatment outside of normal working hours, they will be advised to see their out of hours GP service.

In order to standardise the study prior to commencement the health care professional will receive a Podiatry handbook that will include the following documentation:

- Brief background and aims of the trial 
- Inclusion/exclusion criteria

- How to randomise using the York Trials Unit randomisation service which will provide a patient ID number and treatment group

- Protocols for both treatments

- Documentation/forms used in the trial eg randomisation forms, questionnaires, adverse event reporting, ineligible patient forms

- Discuss possible 'Frequently asked questions' participants may ask about the trial

- Give contact details of the researchers at York University

We will also run a one-day training course.

\section{RECRUITMENT AND RANDOMISATION}

\subsection{Recruitment}

Potential participants for this trial will be identified by the health care professional from either GP referrals or self-referrals received by the Podiatry Schools, podiatry clinics or practice clinic for the treatment of verrucae. In order to facilitate recruitment, after consultation with the local clinics to ensure unmanageable caseloads do not arise, the following strategies may be adopted to increase the number of patients presenting to the clinics:

- Approach GPs in the area requesting them to directly refer patients presenting with a verruca to the podiatry clinic for treatment.

- We will directly advertise for participants eg within GP surgeries and local swimming pools. We will also advertise for participants at local libraries; in local newspapers; University press releases; local radio and tv stations; in the following local NHS departments: dermatology clinics, outpatient departments, A and E and podiatry departments; staff canteens, walk-in centres; at local occupational health departments in large employers near recruiting sites; in large stores such as supermarkets near recruiting sites; at professional development update days run by the podiatry schools and at local private podiatry clinics. 
- Approach secondary schools within the area asking them to send information about the trial to all students. We will also approach local scout, guide, adventure scout, sea and army cadets air training corps and boy's/girl's brigades asking them to give out information about the trial to all members of their group.

- We will approach the University of Bradford and ask if they will post information about the study on their website and noticeboards due to its close proximity to the University of Huddersfield recruiting site.

- We will advertise for participants on local community websites, eg those run by the local Borough and County Councils, local PCT websites and on the EverT trial website.

These patients will be sent an appointment to attend for assessment/treatment along with a recruitment pack and will be given a minimum of 24 hours to consider participation in the trial. The recruitment pack will contain:

- An invitation letter, including contact details for the local health care professional and trial co-ordinator so that potential participants can contact them to discuss any queries they may have regarding the trial.

- An appropriate patient information leaflet(s), an 'adult' information sheet will be sent to participants aged 16 and over. Participants under the age of 16 will receive two information sheets, one designed for children under the age of 16 , the second for their parent/guardian.

- Baseline questionnaire

- Consent form

\subsection{Randomisation}

Those patients that return the baseline questionnaire and indicate that they are willing to take part will be assessed for inclusion in the study by the health care professional when they attend for their appointment. The health care professional will obtain written consent from all patients/and their parent if required, who are willing to participate prior to the patient being randomised. The York Trials Unit will notify the patient's GP of their involvement in 
the study. After consent and before randomisation the health care professional will collect a digital photograph of the verruca.

The health care professional will randomise the patient by either phoning the York Trials Unit remote telephone randomisation service (free phone number) or using the web-based programme. Patients will be allocated to either of the two treatment arms in a 1:1 ratio. Participants will then receive the allocated treatment at that appointment. If at the end of the study the verruca is still present and the participants requires further treatment, the health care professional will consult with the patient as to the best course of action.

Patients who do not wish to take part in the trial or those who wish to opt out will revert to usual care. The health care professional will discuss alternative methods of treatment used within the department with the patient and once a course of treatment has been agreed on, the health care professional will treat and organise further appointments as required.

\subsection{Non recruitment}

The Health care professional will be asked to complete an "Ineligible Patient Form" for those patients who wished to take part in the trial but were ineligible to do so. These forms will be returned to the York Trials Unit. Information collected will be all reasons patients not eligible, DOB, gender, type of wart and date of consideration for trial entry.

\section{OUTCOMES}

\subsection{Primary outcome}

The primary outcome will be complete clearance of all verrucae as observed on digital photographs taken at baseline and 12 weeks and assessed by an independent health care professional. Blind health care professional assessment will also be assessed and will be used if for some reason the digital photograph is not interpretable. 'Clearance' of verrucae will be defined as being the restoration of normal skin upon close inspection, as assessed by the health care professional.

Participants who attend their 12 -week outcome assessment will be sent $£ 20$ to cover any expenses incurred when attending this appointment. 
Participants who do not attend their 12-week outcome assessment appointment will be written to, to determine whether they have a digital camera and if they would be willing to take a photograph of their foot. Those participants who agree to take a photograph will be asked to complete a colour card, which has the participant's ID number on it and the date the photo was taken. The photo will then be sent by email to the York Trials Unit's EverT email account.

Participants may be sent the following reminders

- To attend their final follow up appointment at 12 weeks approximately one week before hand

- To complete follow-up questionnaires, two weeks after the initial questionnaire sent

- At week 7, to stop treatment at week 8 for those assigned to the salicylic acid group

- Weekly to return their tear-off slip when their verruca has gone.

The format of this reminder will either by post, email or text as per the participant's preference.

\subsection{Secondary outcomes}

- Self-reported clearance of verrucae at six months will be assessed by either patient postal or web-based questionnaire according to the participant's preference.

- Self-reported time to clearance of verrucae will be assessed by either patient postal or web-based questionnaire according to the participant's preference.

In addition to this side effects of treatment, pain intensity after treatment, use of painkillers, restrictions to lifestyle due to having the verruca and treatment details will be recorded and assessed by patient postal questionnaire, which will be sent at 1 and 3 weeks after randomisation. The questionnaire will also include a section for the patient to complete and return to the York Trials Unit once the patient believes their verruca has been cured. The format of this questionnaire will be either paper or web based according to the participant's preference. 
Patient satisfaction with the treatment will be recorded by either patient postal or web based questionnaire at 1, 3 and 12 weeks according to the participant's preference. All participants will be sent an unconditional $£ 5$ with the 12 week questionnaire in recognition of their commitment to the study and to cover any expenses incurred in completing the questionnaires.

The influence of prognostic variables on the primary outcome (clearance at 12 weeks) will be investigated. Such variables will include age, type of wart, gender and duration of current wart. The variables to be included will be finalised before any analyses are performed.

Economic evaluation: The Economic evaluation will be carried out from the perspective of the UK health care provider, the National Health Service over a time horizon of 12 weeks and the patient.

Resource data: Data will be collected on the volume of participant access to NHS staff and cost of treatments used during the trial. The number of visits each participant makes to the podiatrist or the health care professional for wart treatment, will be assessed using a participant-completed questionnaires sent at 12 weeks. The use of over the counter verrucae treatments, will be assessed by patient postal questionnaire at 12 weeks.

Health outcomes: We will assess the number of verruca free days for each participant using patient self-reported time to clearance of verrucae.

Cost effectiveness analysis: We will carry out a cost effectiveness analysis as detailed in section 10.4

Data on adverse events will be assessed by the number of visits the participants makes to a doctor or health care professional, and notification of adverse events by the health care professional treating the patient or self-report by the participant 


\section{ETHICAL ARRANGEMENTS}

We are aware that children are considered a vulnerable group. However, we do not anticipate any major ethical issues with the proposed study since both treatments under investigation are currently used within normal practice to treat children with verrucae and Verrugon is licensed as an over the counter treatment.

\subsection{Adverse events/adverse reactions}

The health care professional will routinely record any serious and non-serious adverse events/reactions, which occur during the course of the trial on a serious or non-serious adverse event/reaction form. An assessment of the seriousness, causality and expectedness of the event/reaction will be undertaken. Participants should be asked at each trial visit about hospitalisations, consultations with other medical practitioners, disability or incapacity or whether other relevant adverse events have occurred. The adverse event/adverse reaction reporting period for this trial begins when the patient is randomised into the study and ends 6 months after the date of randomisation. All adverse events and reactions should be followed up until they are resolved or the patient's participation in the trial ends. In addition serious adverse reactions assessed by the investigator as possibly related to the investigational product should continue to be followed even after the patient's participation in the trial is over. Such reactions should be followed until they resolve or until the investigator assesses them as 'chronic' or 'stable'. Appropriate on-going care will be arranged through the appropriate services. Resolution of such events is to be documented on the serious adverse event/reaction form.

Fatal or life-threatening Suspected Unexpected Serious Adverse Reactions (SUSARs) will be recorded and reported to the MHRA, Ethics Committee and Data Monitoring Committee within 7 days of knowledge of such cases. In each case relevant follow-up information should be sought and a report completed as soon as possible. This should be sent to the CA and the Ethics Committee within an additional eight days. All other suspected unexpected serious adverse reactions will be reported to the DMEC, MHRA, and trial sponsor and ethics committee within 15 days of first knowledge. 
Once a year a list of all suspected serious adverse reactions, which have occurred over the period, and a report of the subject' safety will be provided to the MHRA.

The known side effects of treatment associated with treatment with salicylic acid and cryotherapy are: pain, blistering, irritation to the skin, burning sensation, bleeding, scarring, infection and in some cases allergic contact rash may occur in some people.

\subsection{Informing trial participants of possible benefits and risks of intervention}

All trial participants will be provided with a patient information sheet prior to their giving informed consent. The information sheet will outline fully the potential benefits and risks of being involved in the trial. The health care professional will inform the participant if new information comes to light that may affect the participant's willingness to participate in the trial.

\subsection{Informed consent}

Participation in the study will be entirely voluntary and written consent will be sought. All data will be treated with the strictest confidence.

For those participants under the age of 16, the parent/guardian will be asked to give written consent along with assent of the child. The researcher will at all times consider the explicit wish of the minor if they are capable of forming an opinion and assessing the information provided. This will apply not only to the wish of a minor to refuse to take part, but also to withdraw from the trial at any time. Where the parent is competent to decide for their child but unable to read or write, an impartial witness will sign the consent form to say that the information sheet has been read to the parent and verbal consent has been given.

If a clinician feels that the potential participant is unable to give informed consent, then they would not be eligible to take part in the study. 


\subsection{Proposed time period for retention of trial documents}

Paper copies of the relevant trial documentation from the study will be held in a locked room for a period of 9 years at the University of York (i.e, until the youngest participant is aged 21 years), whilst electronic copies will be held indefinitely.

\section{STATISTICAL CONSIDERATONS}

\subsection{SAMPLE SIZE}

The Cochrane systematic review found only one small trial directly comparing the effectiveness of a chemical treatment, salicylic acid, with cryotherapy in patients with warts on their feet alone. This poor quality study found a $58 \%$ cure rate among the patients allocated to cryotherapy compared with $41 \%$ among those treated with salicylic acid. This difference of $17 \%$ was not statistically significant. The overall cure rates from this study are smaller than those observed in two placebo controlled trials of salicylic acid, both of which reported cure rates of $85 \%$ for active treatment, possibly because more resistant verrucae were included in the study comparing cryotherapy with salicylic acid.

In this study we have decided to power the trial to show a $15 \%$ difference in effectiveness. We therefore, will recruit sufficient patients to give us $80 \%$ power $(5 \%$ two sided significance) to show a difference in cure rates of $70 \%$ versus $85 \%$ at 12 weeks after treatment. This will require 120 patients in each group after allowing for a $10 \%$ drop-out rate we will require 133 in each treatment group (i.e. 266 in total). This sample size will also enable us to show that cryotherapy increases the cure rate from $85 \%$ to $97 \%$ (i.e. a $12 \%$ increased cure) for a similar power and significance level.

\subsection{Recruitment}

It is expected that five centres will recruit 3 patients per month, over a recruitment period of 18 months, giving a total of 270 participants. Northampton and Eastbourne Podiatry schools have already agreed to participate in the trial and we have approached Glasgow and Huddersfield Podiatry Schools. We will recruit other podiatry clinics by: 
- Contacting the Heads of the remaining Podiatry Schools

- Networking using the Podiatry Research Forum

- Advertising in "Podiatry Now" for new centres

- Running workshops at the Podiatry Conference

The following sites have agreed to recruit participants: Huddersfield Podiatry School, Brownlow Practice Liverpool, Glasgow Podiatry School/Southern General Hospital, Springfield Surgery Bradford, Islington PCT Dr Mittal Balham London Sheffield PCT, Dr Arthur at Claughton Medical Centre (Birkenhead, Wirral), Dr Ghori at Woodplumpton Road Surgery (Fulwood, Preston) and Dr Rajap at Arlington Road Surgery (Eastbourne).

\section{STATISTICAL ANALYSIS}

\subsection{Primary analysis}

There will be a single principal analysis at the end of the study using $5 \%$ two sided significance tests. We will use 'intention to treat' analysis. All patients will be included in their initially randomised groups whether or not they received their allocated treatment. The primary outcome is complete clearance of all verrucae at 12 weeks. The two treatment groups will be compared using simple proportions of cure or not cured using the Chi squared test. The analysis will be conducted blind to group.

\subsection{Secondary analysis}

As in the primary analysis, all secondary analysis will be by 'intention to treat'. For these secondary outcomes stricter statistical levels of significance will be adopted (i.e. $p=0.01$ ) to reduce the chance of type I error. All analysis will be conducted blind to group.

Data on baseline demographics such as gender, age, type and duration of verrucae, previous treatments will be summarised and descriptive summary statistics provided. For variables with continuous measures we will report the mean and standard deviation, for categorical data we will report numbers and percent. 
The primary analysis will be repeated, but controlling for age, whether or not the wart has been previously treated and type of wart. Should numbers be sufficient, in order to examine whether mosaic warts respond less well to treatment than simple warts, the primary analysis will be repeated, but the type of wart mosaic/simple will be included as a covariate and also an interaction term wart type*treatment will be included. ${ }^{\text {iii }}$

Survival analysis of patient self-reported time to clearance of verrucae, censoring for loss to follow up, will be tested for using Cox regression adjusting for relevant co-variates to be defined before the analysis.

As patients and practitioner are not blinded to treatment, we will carryout a second, sub group analysis, assessing the influence of participant's treatment preference on treatment outcomes and the results of the cost effectiveness analysis.

Data on side effects of and pain intensity during and after treatment, use of painkillers, restrictions to lifestyle due to having a verruca, treatment details, patient satisfaction with treatment, number of warts, will be summarised and descriptive summary statistics provided. For variables with continuous measures we will report the mean and standard deviation, for categorical data we will report numbers and percent.

The number of patients discontinuing treatment prematurely for any reason will be summarised by treatment group and by reasons for discontinuation.

The recurrence/clearance of verrucae at 6 months will be analysed in the same way as the primary outcome measure. 


\subsection{Missing data}

We will try and minimise any missing data with respect to the primary outcome of verrucae clearance within 12 weeks. However if we are unable to ascertain the status of any patients then they will be treated as not having a cleared verrucae in the primary analysis. Sensitivity analyses will be performed considering missing primary outcome data as positive or negative in the different treatment groups. Any missing baseline data will be imputed using appropriate methods before being used in any adjusted analyses.

The incidence of all suspected adverse treatment reactions will be summarised by treatment group.

\subsection{Economic analysis}

We will undertake a cost effectiveness analysis of the treatments. The costs of the two approaches will be collected as part of the study. Costs will be collected by using a patient questionnaire and from clinic records of attendances. For instance we will record the number of attendances to the health care professional both groups have (excluding the final attendance as this a research review). We will then calculate an incremental cost per cured patient at 12 weeks.

The primary economic evaluation will be a cost effectiveness analysis of the trial treatments. The cost of resource use will be calculated for each trial participant using data collected (as described in section 7.2). Staff costs will be calculated using standard NHS costs (Netten A Dennett J, Knight J. Unit costs of Health and Social Care. Caterbury: PSSRU, University of Kent at Canterbury, 2005. Topical treatments will be costed using the BNF (British National Formulary. Number 52 and manufacturer's costs where required. Patient outcome will be measured as verrucae-free days.

The incremental mean difference in costs between the two trial arms and incremental difference in patient outcome will be calculated. There are four potential scenarios: 
1. Cryotherapy is less costly than salicylic acid treatment and leads to better patient outcomes

2. Cryotherapy is more costly than salicylic acid and has worse outcomes

3. Cryotherapy is more costly then salicylic acid but has better (worse) patient outcomes

4. Cryotherapy is less costly than salicylic acid treatment and leads to worse patient outcomes

If we are faced with situation 1 or 2 one treatment clearly dominates the other. That is there is a clear choice about the treatment that is cost-effective. However, if we are faced situation 3 we must weigh up the potential cost implications versus patient benefit to make a decision regarding cost effectiveness. We will do this by relating the incremental mean costs between the two trial arms to the incremental mean outcome as a ratio, the incremental cost effectiveness ratio (ICER). The ICER represents the additional cost per additional verruca free day. A treatment strategy can then be considered cost-effective if the decision maker's willingness to pay for an additional verruca-free day is equal to, or greater, than the ICER Uncertainty regarding the cost effectiveness analysis will be assessed using cost effectiveness acceptability curves.

\subsection{Monitoring of safety}

Data presented to the DMEC will be blind to group allocation at 6 monthly intervals once recruitment has started. The number and type of adverse reaction/event will be reported and compared between the two groups.

\subsection{OTHER CONSIDERATIONS}

A Trial Steering Committee (TSC) will be set up to oversee the conduct of the trial. This will include an independent chair and at least two other independent members, along with the lead investigator and the other study collaborators. They will meet twice a year.

An independent Data Monitoring and Ethics Committee (DMEC) will be set up and will comprise of an independent statistician and podiatrist. The role of the DMEC is to 
immediately see all serious adverse events thought to be treatment related and look at outcome data at six monthly intervals.

\section{PROJECT TIMETABLE}

$1^{\text {st }}$ September 2006: Apply for ethics, research and development (R \& D) and MHRA approval for all sites as required.

Approach other podiatry schools to take part in the trial, apply for ethics and R \& D as appropriate.

October 2006 to Start patient recruitment at Northampton, Eastbourne and new sites as soon as protocol approval/ethics/ R \& D approval are received

Sept 2009 Approach GP practices to refer patients to the podiatry schools and advertise for participants eg at GP surgeries, swimming pools and local secondary schools.

March $2010 \quad$ Final (6 month) follow up questionnaire sent to last participant.

April 2010 Data cleaning, statistical analysis and writing up study findings. Final to June $2010 \quad$ report.

July 2010 Apply to ethics for approval of letter to be sent to trial participants informing them of the study's results.

Send out results of study once approval has been received.

\section{STUDY ORGANISATIONAL STRUCTURE}

\subsection{The York Trials Support Unit (TSU) and trial co-ordination}

The York Trials Support Unit will run the trial, monitor and verify the data and analyse the results. A data coordinator, statistician, data-processing clerk and database programmer for the project will be based in the TSU. 


\section{PUBLICATION POLICY}

The main trial will form the basis of an academic paper in a peer-reviewed journal on its completion. The trial team will also ensure that the results are published in a professional journal in order to ensure access by podiatrists and other health care professionals. The results of the study will be submitted for consideration at the Podiatry Conference.

Dr Mike Curran and Dr Farina Hashmi are members of the Podiatric Research Forum and they will ensure that the results of the trial are disseminated amongst health care professionals.

Participants will receive a summary of the study's findings after obtaining ethical and R \& D approval.

\section{REFERENCES}

\footnotetext{
${ }^{\mathrm{i}}$ McCormick A, Fleming D, Charlton J, Morbidity Statistics from General Practice Fourth National Study 1991-1992 Office of Population Censuses and Surveys Series MB5 No 3. London HMSO

${ }^{\text {ii }}$ Gibbs S, Harvey I, Sterling JC, Stark R. Local treatments for cutaneous warts. British Medical Journal2002;325:461.

iii Pocock SJ. Clinical Trials A practical approach.
} 



\section{Health Technology Assessment programme}

\section{Director,}

Professor Tom Walley, CBE,

Director, NIHR HTA programme, Professor of Clinical Pharmacology,

University of Liverpool

\author{
Deputy Director \\ Professor Hywel Williams, \\ Professor of Dermato-Epidemiology, \\ Centre of Evidence-Based Dermatology, \\ University of Nottingham
}

\section{Prioritisation Group}

\section{Members}

\section{Chair,}

Professor Tom Walley, CBE, Director, NIHR HTA programme, Professor of Clinical Pharmacology, University of Liverpool

Professor Imti Choonara, Professor in Child Health, Academic Division of Child Health, University of Nottingham Chair - Pharmaceuticals Panel

Dr Bob Coates,

Consultant Advisor - Disease

Prevention Panel

Dr Andrew Cook

Consultant Advisor - Intervention

Procedures Panel

Dr Peter Davidson,

Director of NETSCC, Health

Technology Assessment
Dr Nick Hicks,
Consultant Adviser - Diagnostic

Technologies and Screening Panel, Consultant Advisor-Psychological and Community Therapies Panel

Ms Susan Hird,

Consultant Advisor, External

Devices and Physical Therapies Pane

Professor Sallie Lamb,

Director, Warwick Clinical Trials Unit, Warwick Medical School, University of Warwick

Chair - HTA Clinical Evaluation and Trials Board

Professor Jonathan Michaels, Professor of Vascular Surgery, Sheffield Vascular Institute, University of Sheffield Chair - Interventional Procedures Panel
Professor Ruairidh Milne,

Director - External Relations

Dr John Pounsford,

Consultant Physician, Directorate

of Medical Services, North Bristol

NHS Trust

Chair - External Devices and

Physical Therapies Panel

Dr Vaughan Thomas

Consultant Advisor -

Pharmaceuticals Panel, Clinical

Lead - Clinical Evaluation Trials

Prioritisation Group

Professor Margaret Thorogood,

Professor of Epidemiology, Health

Sciences Research Institute,

University of Warwick

Chair - Disease Prevention Panel
Professor Lindsay Turnbull

Professor of Radiology, Centre for the MR Investigations, University of Hull

Chair - Diagnostic Technologies and Screening Panel

Professor Scott Weich,

Professor of Psychiatry, Health

Sciences Research Institute

University of Warwick

Chair - Psychological an

Community Therapies Panel

Professor Hywel Williams,

Director of Nottingham Clinical

Trials Unit, Centre of Evidence-

Based Dermatology, University of

Nottingham

Chair - HTA Commissioning

Board

Deputy HTA Programme Director

\section{HTA Commissioning Board}

\section{Chair}

Professor Hywel Williams,

Professor of Dermato-Epidemiology, Centre of Evidence-Based Dermatology, University of Nottingham
Deputy Chair,

Professor Jon Deeks,

Department of Public Health and

Epidemiology, University of Birmingham
Professor Tom Walley, CBE,

Professor of Clinical Pharmacology, Director,

NIHR HTA programme, University of

Liverpool

\section{Members}

Professor Ann Ashburn,

Professor of Rehabilitation and

Head of Research, Southampton

General Hospital

Professor Peter Brocklehurst, Professor of Women's Health, Institute for Women's Health, University College London

Professor Jenny Donovan, Professor of Social Medicine, University of Bristol

Professor Jonathan Green, Professor and Acting Head of Department, Child and Adolescent Psychiatry, University of Manchester Medical School
Professor John W Gregory,

Professor in Paediatric

Endocrinology, Department of Child Health, Wales School of Medicine, Cardiff University

Professor Steve Halligan, Professor of Gastrointestinal Radiology, University College Hospital, London

Professor Freddie Hamdy, Professor of Urology, Head of Nuffield Department of Surgery, University of Oxford

Professor Allan House, Professor of Liaison Psychiatry, University of Leeds
Dr Martin J Landray, Reader in Epidemiology, Honorary Consultant Physician, Clinical Trial Service Unit, University of Oxford

Professor Stephen Morris, Professor of Health Economics, University College London, Research Department of Epidemiology and Public Health, University College London

Professor Irwin Nazareth, Professor of Primary Care and Head of Department, Departmen of Primary Care and Population Sciences, University College London
Professor E Andrea Nelson, Professor of Wound Healing an Director of Research, School of Healthcare, University of Leeds

Professor John David Norrie, Chair in Clinical Trials and Biostatistics, Robertson Centre for Biostatistics, University of Glasgow

Dr Rafael Perera,

Lecturer in Medical Statisitics, Department of Primary Health Care, University of Oxford 


\section{HTA Commissioning Board (continued)}

Professor Barney Reeves,

Professorial Research Fellow

in Health Services Research,

Department of Clinical Science,

University of Bristo
Professor Martin Underwood,

Professor of Primary Care

Research, Warwick Medical

School, University of Warwick
Professor Marion Walker

Professor in Stroke Rehabilitation,

Associate Director UK Stroke

Research Network, University of

Nottingham
Dr Duncan Young,

Senior Clinical Lecturer and

Consultant, Nuffield Department

of Anaesthetics, University of

Oxford

\section{Observers}

Dr Tom Foulks,

Medical Research Council
Dr Kay Pattison,

Senior NIHR Programme

Manager, Department of Health

\section{HTA Clinical Evaluation and Trials Board}

\section{Chair,}

Professor Sallie Lamb,

Director,

Warwick Clinical Trials Unit

Warwick Medical School,

University of Warwick and Professor of

Rehabilitation,

Nuffield Department of Orthopaedic,

Rheumatology and Musculoskeletal Sciences,

University of Oxford

\section{Deputy Chair,}

Professor Jenny Hewison,

Professor of the Psychology of Health Care,

Leeds Institute of Health Sciences,

University of Leeds
Programme Director,

Professor Tom Walley, CBE,

Director, NIHR HTA programme, Professor of

Clinical Pharmacology, University of Liverpoo

\section{Members}

Professor Keith Abrams, Professor of Medical Statistics, Department of Health Sciences, University of Leicester

Professor Martin Bland, Professor of Health Statistics, Department of Health Sciences, University of York

Professor Jane Blazeby, Professor of Surgery and Consultant Upper GI Surgeon, Department of Social Medicine, University of Bristol

Professor Julia M Brown, Director, Clinical Trials Research Unit, University of Leed

Professor Alistair Burns, Professor of Old Age Psychiatry, Psychiatry Research Group, Schoo of Community-Based Medicine, The University of Manchester \& National Clinical Director for Dementia, Department of Health
Dr Jennifer Burr, Director, Centre for Healthcare Randomised trials (CHART) University of Aberdeen

Professor Linda Davies, Professor of Health Economics, Health Sciences Research Group, University of Manchester

Professor Simon Gilbody, Prof of Psych Medicine and Health Services Research, Department of Health Sciences, University of York

Professor Steven Goodacre Professor and Consultant in Emergency Medicine, School of Health and Related Research, University of Sheffield

Professor Dyfrig Hughes, Professor of Pharmacoeconomics, Centre for Economics and Policy in Health, Institute of Medical and Social Care Research, Bangor University
Professor Paul Jones, Professor of Respiratory Medicine, Department of Cardiac and Vascular Science, St George's Hospital Medical School, University of London

Professor Khalid Khan, Professor of Women's Health and Clinical Epidemiology, Barts and the London School of Medicine, Queen Mary, University of London

Professor Richard J McManus, Professor of Primary Care Cardiovascular Research, Primary Care Clinical Sciences Building,

University of Birmingham

Professor Helen Rodgers, Professor of Stroke Care, Institute for Ageing and Health, Newcastle University

Professor Ken Stein,

Professor of Public Health,

Peninsula Technology Assessment

Group, Peninsula College

of Medicine and Dentistry,

Universities of Exeter and

Plymouth
Professor Jonathan Sterne, Professor of Medical Statistics and Epidemiology, Department of Social Medicine, University of Bristol

Mr Andy Vail,

Senior Lecturer, Health Sciences Research Group, University of Manchester

Professor Clare Wilkinson, Professor of General Practice and Director of Research North Wales Clinical School, Department of Primary Care and Public Health, Cardiff University

Dr Ian B Wilkinson,

Senior Lecturer and Honorary Consultant, Clinical Pharmacology Unit, Department of Medicine, University of Cambridge

\section{Observers}

Ms Kate Law,

Director of Clinical Trials,

Cancer Research UK
Dr Morven Roberts,

Clinical Trials Manager, Health

Services and Public Health

Services Board, Medical Research

Council 


\section{Diagnostic Technologies and Screening Panel}

Members

\section{Chair,}

Professor Lindsay Wilson

Turnbull,

Scientific Director of the

Centre for Magnetic Resonance

Investigations and YCR Professor

of Radiology, Hull Royal Infirmary

Professor Judith E Adams,

Consultant Radiologist,

Manchester Royal Infirmary

Central Manchester \& Manchester

Children's University Hospitals

NHS Trust, and Professor of

Diagnostic Radiology, University

of Manchester

Mr Angus S Arunkalaivanan,

Honorary Senior Lecturer,

University of Birmingham and

Consultant Urogynaecologist

and Obstetrician, City Hospital,

Birmingham

Dr Diana Baralle,

Consultant and Senior Lecturer

in Clinical Genetics, University of

Southampton
Dr Stephanie Dancer,

Consultant Microbiologist,

Hairmyres Hospital, East Kilbride

Dr Diane Eccles,

Professor of Cancer Genetics,

Wessex Clinical Genetics Service,

Princess Anne Hospital

Dr Trevor Friedman,

Consultant Liason Psychiatrist,

Brandon Unit, Leicester General Hospital

Dr Ron Gray,

Consultant, National Perinatal Epidemiology Unit, Institute of Health Sciences, University of Oxford

Professor Paul D Griffiths, Professor of Radiology, Academic

Unit of Radiology, University of Sheffield

Mr Martin Hooper,

Public contributor
Professor Anthony Robert

Kendrick,

Associate Dean for Clinica

Research and Professor of Primary

Medical Care, University of

Southampton

Dr Nicola Lennard,

Senior Medical Officer, MHRA

Dr Anne Mackie,

Director of Programmes, UK

National Screening Committee,

London

Mr David Mathew,

Public contributor

Dr Michael Millar,

Consultant Senior Lecturer in

Microbiology, Department of

Pathology \& Microbiology, Barts

and The London NHS Trust, Royal

London Hospital

Dr Stuart Smellie,

Consultant in Clinical Pathology,

Bishop Auckland General Hospital

Ms Jane Smith,

Consultant Ultrasound

Practitioner, Leeds Teaching

Hospital NHS Trust, Leeds

Dr Allison Streetly,

Programme Director, NHS Sickle

Cell and Thalassaemia Screening

Programme, King's College School of Medicine

Dr Matthew Thompson,

Senior Clinical Scientist and GP

Department of Primary Health

Care, University of Oxford

Dr Alan J Williams,

Consultant Physician, General and

Respiratory Medicine, The Royal

Bournemouth Hospital

\section{Observers}

\section{Dr Tim Elliott,}

Team Leader, Cancer Screening,

Department of Health

Dr Joanna Jenkinson,

Board Secretary, Neurosciences

and Mental Health Board

(NMHB), Medical Research

Council
Mrs Una Rennard,

Public contributor
Professor Julietta Patnick,

Director, NHS Cancer Screening

Programme, Sheffield

Dr Kay Pattison,

Senior NIHR Programme

Manager, Department of Health
Professor Tom Walley, CBE, Director, NIHR HTA

programme, Professor of Clinical

Pharmacology, University of

Liverpool
Dr Ursula Wells,

Principal Research Officer, Policy Research Programme, Department of Health

\section{Disease Prevention Panel}

Members

\begin{tabular}{|c|c|c|c|}
\hline $\begin{array}{l}\text { Chair, } \\
\text { Professor Margaret Thorogood, } \\
\text { Professor of Epidemiology, } \\
\text { University of Warwick Medical } \\
\text { School, Coventry }\end{array}$ & $\begin{array}{l}\text { Professor Cathy Jackson, } \\
\text { Professor of Primary Care } \\
\text { Medicine, Bute Medical School, } \\
\text { University of St Andrews } \\
\text { Dr Russell Jago, }\end{array}$ & $\begin{array}{l}\text { Professor Irwin Nazareth, } \\
\text { Professor of Primary Care and } \\
\text { Director, Department of Primary } \\
\text { Care and Population Sciences, } \\
\text { University College London }\end{array}$ & $\begin{array}{l}\text { Dr Kenneth Robertson, } \\
\text { Consultant Paediatrician, Royal } \\
\text { Hospital for Sick Children, } \\
\text { Glasgow }\end{array}$ \\
\hline $\begin{array}{l}\text { Dr Robert Cook, } \\
\text { Clinical Programmes Director, } \\
\text { Bazian Ltd, London } \\
\text { Dr Colin Greaves, }\end{array}$ & $\begin{array}{l}\text { Senior Lecturer in Exercise, } \\
\text { Nutrition and Health, Centre } \\
\text { for Sport, Exercise and Health, } \\
\text { University of Bristol }\end{array}$ & $\begin{array}{l}\text { Dr Richard Richards, } \\
\text { Assistant Director of Public } \\
\text { Health, Derbyshire County } \\
\text { Primary Care Trust }\end{array}$ & $\begin{array}{l}\text { Associate Director, Centre for } \\
\text { Public Health Excellence, NICE } \\
\text { Mrs Jean Thurston, } \\
\text { Public contributor }\end{array}$ \\
\hline $\begin{array}{l}\text { Senior Research Fellow, Peninsula } \\
\text { Medical School (Primary Care) } \\
\text { Mr Michael Head, } \\
\text { Public contributor }\end{array}$ & $\begin{array}{l}\text { Dr Julie Mytton, } \\
\text { Consultant in Child Public Health, } \\
\text { NHS Bristol }\end{array}$ & $\begin{array}{l}\text { Professor Ian Roberts, } \\
\text { Professor of Epidemiology and } \\
\text { Public Health, London School of } \\
\text { Hygiene \& Tropical Medicine }\end{array}$ & $\begin{array}{l}\text { Professor David Weller, } \\
\text { Head, School of Clinical Science } \\
\text { and Community Health, } \\
\text { University of Edinburgh }\end{array}$ \\
\hline
\end{tabular}

Observers

Ms Christine McGuire,

Research \& Development,

Department of Health
Dr Kay Pattison,

Senior NIHR Programme

Manager, Department of Health
Professor Tom Walley, CBE,

Director, NIHR HTA

programme, Professor of Clinical

Pharmacology, University of

Liverpool 


\section{External Devices and Physical Therapies Panel}

Members

\section{Chair,}

Dr John Pounsford,

Consultant Physician North Bristol

NHS Trust

Deputy Chair,

Professor E Andrea Nelson,

Reader in Wound Healing and

Director of Research, University

of Leeds

Professor Bipin Bhakta,

Charterhouse Professor in

Rehabilitation Medicine,

University of Leeds

Mrs Penny Calder,

Public contributor
Dr Dawn Carnes,

Senior Research Fellow, Barts and the London School of Medicine and Dentistry

Dr Emma Clark,

Clinician Scientist Fellow \& Cons.

Rheumatologist, University of Bristol

Mrs Anthea De Barton-Watson,

Public contributor

Professor Nadine Foster, Professor of Musculoskeletal Health in Primary Care Arthritis Research, Keele University
Dr Shaheen Hamdy,

Clinical Senior Lecturer and

Consultant Physician, University

of Manchester

Professor Christine Norton,

Professor of Clinical Nursing

Innovation, Bucks New University

and Imperial College Healthcare

NHS Trust

Dr Lorraine Pinnigton,

Associate Professor in

Rehabilitation, University of

Nottingham

Dr Kate Radford,

Senior Lecturer (Research),

University of Central Lancashire
Mr Jim Reece,

Public contributor

Professor Maria Stokes

Professor of Neuromusculoskeletal

Rehabilitation, University of

Southampton

Dr Pippa Tyrrell,

Senior Lecturer/Consultant

Salford Royal Foundation

Hospitals' Trust and University of Manchester

Dr Nefyn Williams,

Clinical Senior Lecturer, Cardiff

University

\section{Observers}

Dr Kay Pattison,

Senior NIHR Programme

Manager, Department of Health
Dr Morven Roberts,

Clinical Trials Manager, Health

Services and Public Health

Services Board, Medical Research

Council
Professor Tom Walley, CBE,

Director, NIHR HTA

programme, Professor of Clinical

Pharmacology, University of

Liverpool
Dr Ursula Wells,

Principal Research Officer, Policy

Research Programme, Department of Health

\section{Interventional Procedures Panel}

Members

\section{Chair, \\ Professor Jonathan Michaels, Professor of Vascular Surgery,}

University of Sheffield

\section{Deputy Chair,}

Mr Michael Thomas,

Consultant Colorectal Surgeon, Bristol Royal Infirmary

Mrs Isabel Boyer,

Public contributor

Mr Sankaran Chandra Sekharan, Consultant Surgeon, Breast Surgery, Colchester Hospital University NHS Foundation Trust

Professor Nicholas Clarke,

Consultant Orthopaedic Surgeon,

Southampton University Hospitals NHS Trust

Ms Leonie Cooke Public contributor

\section{Mr Seumas Eckford,}

Consultant in Obstetrics \&

Gynaecology, North Devon

District Hospital

Professor Sam Eljamel,

Consultant Neurosurgeon

Ninewells Hospital and Medical

School, Dundee

Dr Adele Fielding,

Senior Lecturer and Honorary

Consultant in Haematology,

University College London

Medical School

Dr Matthew Hatton,

Consultant in Clinical Oncology,

Sheffield Teaching Hospital

Foundation Trust

Dr John Holden,

General Practitioner, Garswood

Surgery, Wigan
Dr Fiona Lecky,

Senior Lecturer/Honorary

Consultant in Emergency

Medicine, University of

Manchester/Salford Royal

Hospitals NHS Foundation Trust

Dr Nadim Malik,

Consultant Cardiologist/Honorary

Lecturer, University of Manchester

Mr Hisham Mehanna,

Consultant \& Honorary Associate

Professor, University Hospitals

Coventry \& Warwickshire NHS Trust

Dr Jane Montgomery,

Consultant in Anaesthetics and

Critical Care, South Devon

Healthcare NHS Foundation Trust

Professor Yit Chiun Yang,

Consultant Ophthalmologist,

Royal Wolverhampton Hospitals

NHS Trust

\section{Observers}

Dr Kay Pattison,

Senior NIHR Programme

Manager, Department of Health
Dr Morven Roberts,

Clinical Trials Manager, Health

Services and Public Health

Services Board, Medical Research

Council
Professor Tom Walley, CBE,

Director, NIHR HTA

programme, Professor of Clinical

Pharmacology, University of

Liverpool
Dr Ursula Wells,

Principal Research Officer, Policy

Research Programme, Departmen of Health 


\section{Pharmaceuticals Panel}

Members

\section{Chair,}

Professor Imti Choonara

Professor in Child Health,

University of Nottingham

\section{Deputy Chair,}

Dr Yoon K Loke

Senior Lecturer in Clinical

Pharmacology, University of East

Anglia

Dr Martin Ashton-Key,

Medical Advisor, National

Commissioning Group, NHS

London

Dr Peter Elton,

Director of Public Health, Bury

Primary Care Trust

Dr Ben Goldacre,

Research Fellow, Epidemiology

London School of Hygiene and

Tropical Medicine
Dr James Gray,

Consultant Microbiologist,

Department of Microbiology,

Birmingham Children's Hospital

NHS Foundation Trust

Dr Jurjees Hasan,

Consultant in Medical Oncology,

The Christie, Manchester

Dr Carl Heneghan,

Deputy Director Centre for

Evidence-Based Medicine and

Clinical Lecturer, Department of

Primary Health Care, University of Oxford

\section{Dr Dyfrig Hughes,}

Reader in Pharmacoeconomics and Deputy Director, Centre for Economics and Policy in Health, IMSCaR, Bangor University
Dr Maria Kouimtzi,

Pharmacy and Informatics

Director, Global Clinical Solutions,

Wiley-Blackwell

Professor Femi Oyebode,

Consultant Psychiatrist and Head

of Department, University of

Birmingham

Dr Andrew Prentice,

Senior Lecturer and Consultant

Obstetrician and Gynaecologist

The Rosie Hospital, University of

Cambridge

Ms Amanda Roberts,

Public contributor

Dr Gillian Shepherd,

Director, Health and Clinical

Excellence, Merck Serono Ltd
Mrs Katrina Simister

Assistant Director New Medicines,

National Prescribing Centre,

Liverpool

Professor Donald Singer,

Professor of Clinical

Pharmacology and Therapeutics,

Clinical Sciences Research

Institute, CSB, University of

Warwick Medical School

Mr David Symes,

Public contributor

Dr Arnold Zermansky,

General Practitioner, Senior

Research Fellow, Pharmacy

Practice and Medicines

Management Group, Leeds

University

\section{Observers}

\section{Dr Kay Pattison,}

Senior NIHR Programme

Manager, Department of Health

Mr Simon Reeve,

Head of Clinical and Cost-

Effectiveness, Medicines,

Pharmacy and Industry Group,

Department of Health
Dr Heike Weber,

Programme Manager, Medical

Research Council

Professor Tom Walley, CBE,

Director, NIHR HTA

programme, Professor of Clinical

Pharmacology, University of

Liverpool
Dr Ursula Wells,

Principal Research Officer, Policy Research Programme, Department of Health

\section{Psychological and Community Therapies Panel}

\section{Members}

\begin{tabular}{ll}
\hline Chair, & Mrs Val Carlill, \\
Professor Scott Weich, & Public contribut
\end{tabular}

Professor of Psychiatry, University

of Warwick, Coventry

\section{Deputy Chair,}

\section{Dr Howard Ring,}

Consultant \& University Lecture in Psychiatry, University of

Cambridge

Professor Jane Barlow, Professor of Public Health in the Early Years, Health Sciences Research Institute, Warwick Medical Schoo

Dr Sabyasachi Bhaumik Consultant Psychiatrist Leicestershire Partnership NHS Trust

\section{Public contributor}

Dr Steve Cunningham,

Consultant Respiratory

Paediatrician, Lothian Health

Board

Dr Anne Hesketh,

Senior Clinical Lecturer in Speech and Language Therapy, University of Manchester

Dr Peter Langdon,

Senior Clinical Lecturer, Schoo of Medicine, Health Policy and Practice, University of East Anglia

Dr Yann Lefeuvre,

GP Partner, Burrage Road Surgery, London
Dr Jeremy J Murphy,

Consultant Physician and

Cardiologist, County Durham and

Darlington Foundation Trus

Dr Richard Neal,

Clinical Senior Lecturer in General Practice, Cardiff University

Mr John Needham,

Public contributor

Ms Mary Nettle,

Mental Health User Consultant

Professor John Potter,

Professor of Ageing and Stroke Medicine, University of East

Anglia

Dr Greta Rait,

Senior Clinical Lecturer and

General Practitioner, University

College London
Dr Paul Ramchandani,

Senior Research Fellow/Cons.

Child Psychiatrist, University of Oxford

Dr Karen Roberts,

Nurse/Consultant, Dunston Hill Hospital, Tyne and Wear

Dr Karim Saad,

Consultant in Old Age Psychiatry, Coventry and Warwickshire Partnership Trust

Dr Lesley Stockton,

Lecturer, School of Health

Sciences, University of Liverpool

Dr Simon Wright,

GP Partner, Walkden Medical

Centre, Manchester

\section{Observers}

Dr Kay Pattison,

Senior NIHR Programme

Manager, Department of Health
Dr Morven Roberts,

Clinical Trials Manager, Health

Services and Public Health

Services Board, Medical Research

Council
Professor Tom Walley, CBE, Director, NIHR HTA programme, Professor of Clinical Pharmacology, University of Liverpool
Dr Ursula Wells,

Principal Research Officer, Policy Research Programme, Department of Health 


\section{Expert Advisory Network}

\section{Members}

Professor Douglas Altman, Professor of Statistics in Medicine, Centre for Statistics in Medicine, University of Oxford

Professor John Bond, Professor of Social Gerontology \& Health Services Research, University of Newcastle upon Tyne

Professor Andrew Bradbury, Professor of Vascular Surgery, Solihull Hospital, Birmingham

Mr Shaun Brogan,

Chief Executive, Ridgeway Primary Care Group, Aylesbury

Mrs Stella Burnside OBE, Chief Executive, Regulation and Improvement Authority, Belfast

Ms Tracy Bury,

Project Manager, World

Confederation of Physical Therapy, London

Professor Iain T Cameron,

Professor of Obstetrics and Gynaecology and Head of the School of Medicine, University of Southampton

Professor Bruce Campbell, Consultant Vascular \& General Surgeon, Royal Devon \& Exeter Hospital, Wonford

Dr Christine Clark Medical Writer and Consultant Pharmacist, Rossendale

Professor Collette Clifford, Professor of Nursing and Head of Research, The Medical School, University of Birmingham

Professor Barry Cookson, Director, Laboratory of Hospital Infection, Public Health Laboratory Service, London

Dr Carl Counsell,

Clinical Senior Lecturer in Neurology, University of Aberdeen

Professor Howard Cuckle, Professor of Reproductive Epidemiology, Department of Paediatrics, Obstetrics \& Gynaecology, University of Leeds

Professor Carol Dezateux

Professor of Paediatric

Epidemiology, Institute of Child

Health, London

Mr John Dunning,

Consultant Cardiothoracic

Surgeon, Papworth Hospital NHS

Trust, Cambridge

Mr Jonothan Earnshaw,

Consultant Vascular Surgeon,

Gloucestershire Royal Hospital,

Gloucester
Professor Martin Eccles,

Professor of Clinical Effectiveness,

Centre for Health Services

Research, University of Newcastle upon Tyne

Professor Pam Enderby,

Dean of Faculty of Medicine,

Institute of General Practice

and Primary Care, University of

Sheffield

Professor Gene Feder,

Professor of Primary Care

Research \& Development, Centre

for Health Sciences, Barts and Th

London School of Medicine and

Dentistry

Mr Leonard R Fenwick,

Chief Executive, Freeman

Hospital, Newcastle upon Tyne

Mrs Gillian Fletcher,

Antenatal Teacher and Tutor and

President, National Childbirth

Trust, Henfield

Professor Jayne Franklyn,

Professor of Medicine, University of Birmingham

Mr Tam Fry,

Honorary Chairman, Child

Growth Foundation, London

Professor Fiona Gilbert,

Consultant Radiologist and NCRN

Member, University of Aberdeen

Professor Paul Gregg,

Professor of Orthopaedic Surgical

Science, South Tees Hospital NHS

Trust

Bec Hanley,

Co-director, TwoCan Associates,

West Sussex

Dr Maryann L Hardy,

Senior Lecturer, University of

Bradford

Mrs Sharon Hart,

Healthcare Management

Consultant, Reading

Professor Robert E Hawkins,

CRC Professor and Director of

Medical Oncology, Christie CRC

Research Centre, Christie Hospital

NHS Trust, Manchester

Professor Richard Hobbs,

Head of Department of Primary

Care \& General Practice,

University of Birmingham

Professor Alan Horwich,

Dean and Section Chairman,

The Institute of Cancer Research, London

Professor Allen Hutchinson, Director of Public Health and Deputy Dean of ScHARR,

University of Sheffield
Professor Peter Jones,

Professor of Psychiatry, University of Cambridge, Cambridge

Professor Stan Kaye,

Cancer Research UK Professor of Medical Oncology, Royal Marsden Hospital and Institute of Cancer Research, Surrey

Dr Duncan Keeley,

General Practitioner (Dr Burch \& Ptnrs), The Health Centre, Thame

Dr Donna Lamping,

Research Degrees Programme

Director and Reader in

Psychology, Health Services

Research Unit, London School of

Hygiene and Tropical Medicine,

London

Professor James Lindesay,

Professor of Psychiatry for the

Elderly, University of Leicester

Professor Julian Little,

Professor of Human Genome

Epidemiology, University of

Ottawa

Professor Alistaire McGuire,

Professor of Health Economics,

London School of Economics

Professor Neill McIntosh

Edward Clark Professor of Child

Life and Health, University of Edinburgh

Professor Rajan Madhok,

Consultant in Public Health, South

Manchester Primary Care Trust

Professor Sir Alexander Markham Director, Molecular Medicine Unit, St James's University

Hospital, Leeds

Dr Peter Moore

Freelance Science Writer, Ashtead

Dr Andrew Mortimore,

Public Health Director,

Southampton City Primary Care

Trust

Dr Sue Moss,

Associate Director, Cancer

Screening Evaluation Unit,

Institute of Cancer Research,

Sutton

Professor Miranda Mugford, Professor of Health Economics and Group Co-ordinator, University of East Anglia

Professor Jim Neilson, Head of School of Reproductive \& Developmental Medicine and Professor of Obstetrics and Gynaecology, University of Liverpool
Mrs Julietta Patnick,

Director, NHS Cancer Screening Programmes, Sheffield

Professor Robert Peveler, Professor of Liaison Psychiatry, Royal South Hants Hospital, Southampton

Professor Chris Price,

Director of Clinical Research,

Bayer Diagnostics Europe, Stoke Poges

Professor William Rosenberg,

Professor of Hepatology and

Consultant Physician, University of Southampton

Professor Peter Sandercock, Professor of Medical Neurology, Department of Clinical

Neurosciences, University of Edinburgh

Dr Philip Shackley,

Senior Lecturer in Health

Economics, Sheffield Vascular

Institute, University of Sheffield

Dr Eamonn Sheridan,

Consultant in Clinical Genetics, St James's University Hospital, Leeds

Dr Margaret Somerville,

Director of Public Health

Learning, Peninsula Medical School, University of Plymouth

Professor Sarah Stewart-Brown Professor of Public Health,

Division of Health in the Community, University of Warwick, Coventry

Dr Nick Summerton, GP Appraiser and Codirector, Research Network, Yorkshire Clinical Consultant, Primary Care and Public Health, University of Oxford

Professor Ala Szczepura, Professor of Health Service Research, Centre for Health Services Studies, University of Warwick, Coventry

Dr Ross Taylor,

Senior Lecturer, University of Aberdeen

Dr Richard Tiner,

Medical Director, Medical

Department, Association of the British Pharmaceutical Industry

Mrs Joan Webster,

Consumer Member, Southern

Derbyshire Community Health Council

Professor Martin Whittle, Clinical Co-director, National Co-ordinating Centre for Women's and Children's Health, Lymington 



\section{Feedback}

The HTA programme and the authors would like to know your views about this report.

The Correspondence Page on the HTA website (www.hta.ac.uk) is a convenient way to publish your comments. If you prefer, you can send your comments to the address below, telling us whether you would like us to transfer them to the website.

We look forward to hearing from you.

NETSCC, Health Technology Assessment

Alpha House

University of Southampton Science Park

Southampton SO16 7NS, UK

Email: hta@hta.ac.uk

www.hta.ac.uk 